Aus der Klinik für Unfallchirurgie und Orthopädie

(Prof. Dr. med. K. M. Stürmer)

der Medizinischen Fakultät der Universität Göttingen

\title{
Kinematik der Wirbelsäule \\ Literaturanalyse
}

INAUGURAL-DISSERTATION

zur Erlangung des Doktorgrades

für Zahnheilkunde

der Medizinischen Fakultät der

Georg-August-Universität zu Göttingen

vorgelegt von

Conrad Raschke

aus

Magdeburg

Göttingen 2016 
Dekan:

Prof. Dr. rer. nat. H. K. Kroemer

I. Berichterstatter/in:

II. Berichterstatter/in: Prof. Dr. med. A.-K. Hell

III. Berichterstatter/in:
PD Dr. med. M.M.Wachowski

Tag der mündlichen Prüfung: 15.08 .2016 


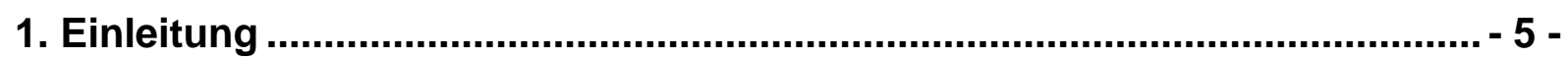

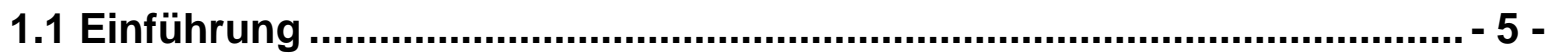

1.2 Funktionelle Anatomie der Wirbelsäule ................................................ - 8 -

1.3 Physikalische Grundlagen räumlicher Bewegungsvorgänge starrer

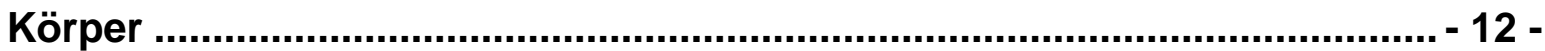

1.3.1 Kinematische Schraubung .............................................................. 12 -

1.3.2 Kraftschraube .............................................................................. 15 -

1.4 Allgemeines zur biomechanischen Charakterisierung der

Wirbelsegmentbewegung ....................................................................... - 16 -

1.4.1 Anatomie eines Wirbelsegmentes und daran orientierte anschaulich definierte Begriffe ......................................................................... - 16 -

1.5 Grundlagen der In-vitro-Untersuchung der Wirbelsegmentbewegung - 17 -

1.5.1 Wahl des angreifenden Kraftsystems.............................................. - 17 -

1.5.2 Voraussetzungen zur Messung der Segmentbewegung ...................... - 17 -

1.5.3 Kennlinien der Segmente ................................................................ - 18 -

1.5.4 Im Schrifttum verwendete Begriffe zur Beschreibung der Rotations-/

Schraubachse ..................................................................................... - 20 -

1.6 Kriterien zur Beurteilung der in der vorliegenden Arbeit zu untersuchenden Studien: ........................................................................ - 21 -

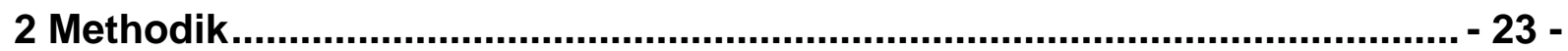

3 Testverfahren/ Messergebnisse ................................................................ 26 -

3.1 Zielstellungen der untersuchten Studien ........................................... - 26 -

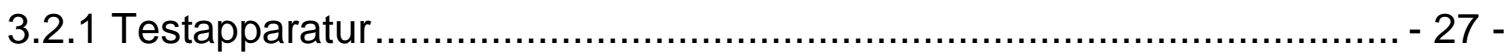

3.2.2 Drehmomentapplikation .............................................................. 27 -

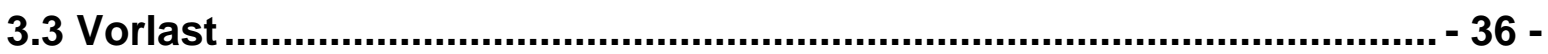

3.4 Verwendete Präparate....................................................................... 43 -

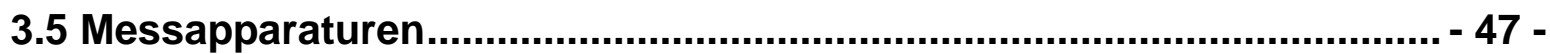

3.5.1 Röntgenbildanalysen.................................................................... 48 -

3.5.2 Optische Verfahren ................................................................ - 48 -

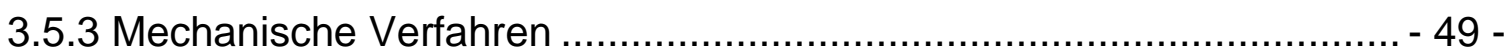

3.5.4 Messung über Ultraschall................................................................ - 49 -

3.6 Angaben zu Rotations-/Schraubachsen der untersuchten Studien ..... - 54 3.7 Vergleich der Lokalisation errechneter Rotations-/Schraubachsen intakter zervikaler und lumbaler Segmente ....................................................... 59 -

3.7.1 Zervikale Segmente …………............................................. 59 -

3.7.2 Lumbale Segmente ...................................................................... 65 -

3.8 Angaben zum Bewegungsausmaß und Gelenkkräften in den untersuchten

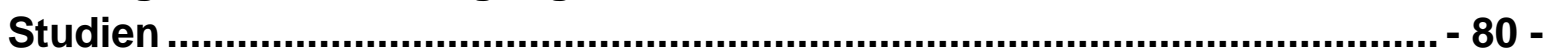

3.8.1 Bewegungsausmaß (ROM) ........................................................ 80 -

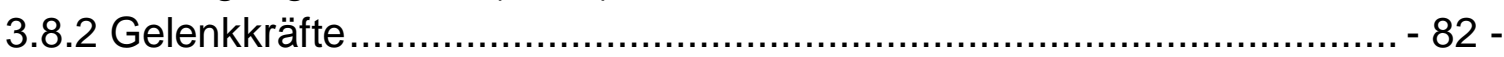

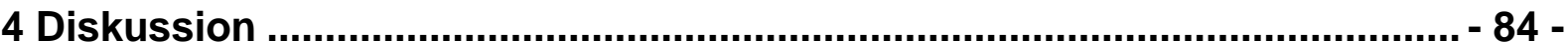

4.1 Zielstellungen der untersuchten Studien ........................................... - 84 -

4.2 Testapparatur und Drehmomentapplikation ......................................... - 84 - 


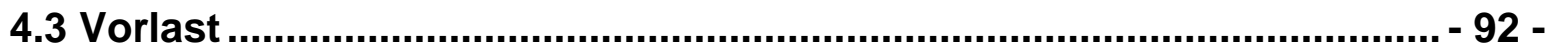

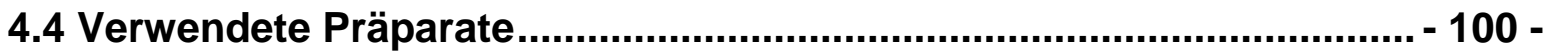

4.5 Messapparaturen.......................................................................... - 102 -

4.6 Angaben zu Rotations-/Schraubachsen der untersuchten Studien ... - 103 4.7 Vergleich der Lokalisation ermittelter Rotations-/Schraubachsen ausgewählter Studien $107-$ 4.8 Angaben zum Bewegungsausmaß und Gelenkkräften in den untersuchten Studien 109 -

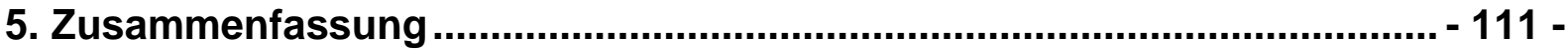

6. Literaturverzeichnis.................................................................................. 113 -

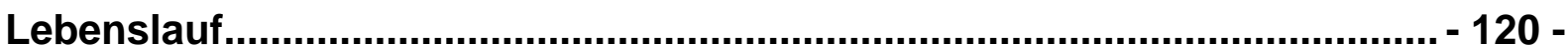




\section{Abbildungsverzeichnis}

\begin{tabular}{|c|c|c|}
\hline Abb. 1 & S. 13 & Weg zwischen zwei Orten verläuft nicht unbedingt entlang einer \\
\hline Abb. 2 & S. 13 & $\begin{array}{l}\text { Bewegung eines Körpers von der Position } 1 \text { in Position 2, unterteilt } \\
\text { in eine Rotationsbewegung bzw. eine Translationsbewegung } \\
\text { (Bockermann 2004, S.20) }\end{array}$ \\
\hline Abb. 3 & S. 14 & $\begin{array}{l}\text { Darstellung Schraubachsen für die axiale Rotation (Bockermann } \\
\text { 2004, S. 48). }\end{array}$ \\
\hline Abb. 4 & S. 15 & Momentane Lage einer Schraubachse \\
\hline Abb. 5 & S. 16 & $\begin{array}{l}\text { Koordinatensystem und eine mögliche Kraftschraube bei axialer } \\
\text { Rotation, (Mansour 2001, S. 74) }\end{array}$ \\
\hline Abb. 6 & S. 19 & $\begin{array}{l}\text { Möglicher Verlauf einer Bewegung. Darstellung einer Hysteresflä- } \\
\text { che }\end{array}$ \\
\hline Abb. 7 & S. 20 & $\begin{array}{l}\text { Darstellung einer Drehwinkel-/Drehmomentkurve mit Einteilung des } \\
\text { ROM in NZ und EZ }\end{array}$ \\
\hline Abb. 8 & S. 29 & $\begin{array}{l}\text { Häufig verwendete Apparatur (aus Crawford et al. 1995, S. } 2098 \\
\text { mit Erlaubnis von Lipincott Williams and Wilkins/ Wolters Kluwer } \\
\text { Health) }\end{array}$ \\
\hline Abb. 9 & S. 31 & Beispiel einer Versuchsapparatur (aus Mansour 2001, S. 73) \\
\hline Abb. 10 & S. 37 & $\begin{array}{l}\text { Beispiel einer Versuchsapparatur (McNally et al. 2012, } \\
\text { S.613; mit freundlicher Erlaubnis von Springer Science } \\
\text { and Business Media) }\end{array}$ \\
\hline Abb. 11 & S. 40 & $\begin{array}{l}\text { Formen der Vorlastapplikation (Patwardhan et al. 1999, } \\
\text { S. 1004, mit Erlaubnis von Lipincott Williams and Wil- } \\
\text { kins/ Wolters Kluwer Health) }\end{array}$ \\
\hline Abb. 12 & S. 40 & $\begin{array}{l}\text { Beispiel einer Versuchsapparatur (Haberl et al. 2004, S. 562, mit } \\
\text { freundlicher Erlaubnis von Springer Science and Business Media) }\end{array}$ \\
\hline Abb. 13 & S. 72 & $\begin{array}{l}\text { Darstellung HAM bei axialer Rotation (Takigawa et al. 2010, mit } \\
\text { Erlaubnis von Lipincott Williams and Wilkins/ Wolters Kluwer } \\
\text { Health) }\end{array}$ \\
\hline Abb. 14 & S. 72 & $\begin{array}{l}\text { Darstellung Achsschar bei axialer Rotation (Kettler et al. 2004, mit } \\
\text { freundlicher Erlaubnis von Springer Science and Business Media) }\end{array}$ \\
\hline Abb. 15 & S. 73 & $\begin{array}{l}\text { Darstellung Rastpolkurven in Wachowski et al. 2009a bei axialer } \\
\text { Rotation (Wachowski et al. 2009a, mit Erlaubnis von Elsevier) }\end{array}$ \\
\hline Abb. 16 & S. 74 & $\begin{array}{l}\text { Schnittpunkt der HAM mit Transversalebene (Haberl et al. 2004, } \\
\text { mit freundlicher Erlaubnis von Springer Science and Business Me- } \\
\text { dia) }\end{array}$ \\
\hline Abb. 17 & S. 74 & $\begin{array}{l}\text { Lage und Orientierung HAM in Haberl et al. (Haberl et al 2004, mit } \\
\text { freundlicher Erlaubnis von Springer Science and Business Media) }\end{array}$ \\
\hline Abb. 18 & S. 76 & $\begin{array}{l}\text { Darstellung HAM bei lateraler Beugung (Takigawa et al. 2010, mit } \\
\text { Erlaubnis von Lipincott Williams and Wilkins/ Wolters Kluwer } \\
\text { Health) }\end{array}$ \\
\hline Abb. 19 & S. 76 & $\begin{array}{l}\text { Darstellung Achsschar bei lateraler Beugung in L4-L5 Segmenten } \\
\text { (Kettler et al. 2004, mit freundlicher Erlaubnis von Springer Science } \\
\text { and Business Media) }\end{array}$ \\
\hline Abb. 20 & S. 77 & $\begin{array}{l}\text { Darstellung Rastpolkurven in Wachowski et al. 2009a bei lateraler } \\
\text { Beugung (Wachowski et al. 2009a, mit Erlaubnis von Elsevier) }\end{array}$ \\
\hline Abb. 21 & S. 79 & $\begin{array}{l}\text { Darstellung HAM bei Flexion- Extension } \\
\text { (Takigawa et al. 2010, mit Erlaubnis von Lipincott Willi- } \\
\text { ams and Wilkins/ Wolters Kluwer Health) }\end{array}$ \\
\hline
\end{tabular}


\begin{tabular}{|l|l}
\hline Abb. 22 S. 79 & Darstellung Achsschar bei Flexion- Extension (Kettler et al. 2004,
\end{tabular} mit freundlicher Erlaubnis von Springer Science and Business Media)

Abb. 23 S. $80 \quad$ Darstellung Rastpolkurven bei Flexion- Extension (Wachowski et al. 2009a, mit Erlaubnis von Elsevier)

Tabellenverzeichnis

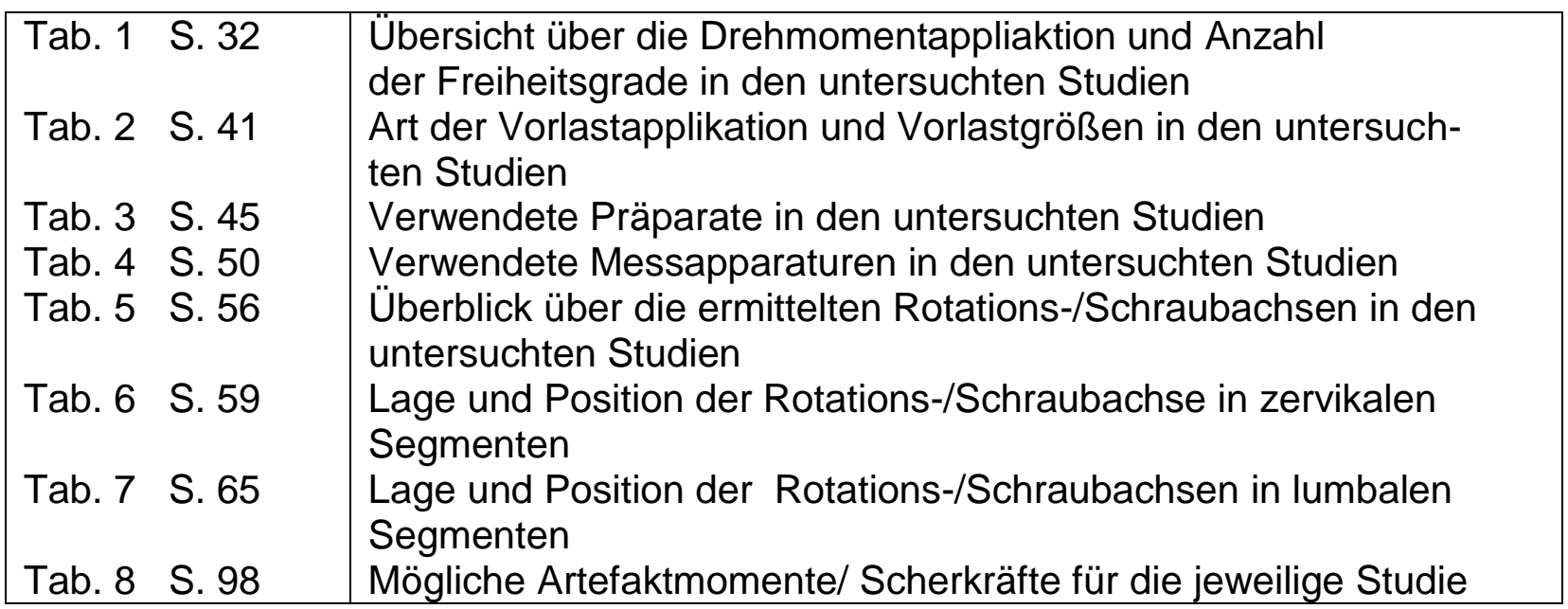




\section{Einleitung}

\subsection{Einführung}

Der Begriff „Rückenschmerz“ ist ein weitgefasster Begriff. Zahlreiche Schlagwörter wie „HWS-Syndrom“ (Halswirbelsäulensyndrom), „Kreuzschmerz“ oder „LBP“ (lower back pain) haben sich gesellschaftlich etabliert und versuchen, die teils spezifischen und unspezifischen Symptome näher zu beschreiben. Die Lebenszeitprävalenz von Rückenschmerzen liegt in Deutschland bei 85,5\% (Schmidt et al. 2007), weswegen die Problematik unweigerlich eine hohe medizinische Bedeutung hat. Die hohe Prävalenz schlägt sich auch in finanziellen und wirtschaftlichen Konsequenzen nieder. So wurde in einer Studie 2009 berechnet, dass direkte und indirekte, durch Rückenschmerzen verursachte Kosten, in Deutschland durchschnittlich 1322 Euro pro Patient und Jahr betragen (Wenig et al. 2009). Das Statistische Bundesamt gibt an, dass Dorsopathien im Jahr 2008 direkte Kosten in Höhe von 9.043.000.000 Euro verursachten (Statistisches Bundesamt 2010). Auch die indirekten volkswirtschaftlichen Folgekosten müssen Beachtung finden, da Rückenschmerzen häufig zu Arbeitsunfähigkeit oder Frühverrentung führen. So beziffert das Statistische Bundesamt den Verlust an Erwerbstätigkeitsjahren in Deutschland aufgrund von Dorsopathien für das Jahr 2008 mit 247.000 Jahren (Statistisches Bundesamt 2010). Neben dem hohen individuellen Leid des Patienten sind also auch die enorm hohen Folgekosten zu beachten. Dies macht es erforderlich, die Ursachen und gegenwärtigen Behandlungsmöglichkeiten der genannten Problematik näher zu beleuchten:

Häufig liegen den schmerzhaften Symptomen degenerative Prozesse der Wirbelsegmente zu Grunde. Diese werden oftmals mit Hilfe konservativer Therapieverfahren in Form von Bewegungs- und Sporttherapie, Chirotherapie, Ergotherapie oder Medikamenten behandelt (Raspe 2012). Dabei handelt es sich jedoch um eine rein symptomatische Behandlung, die daher nicht dem medizinischen Anspruch einer auf die Ursache bezogenen Therapie entspricht.

So sind auch operative Eingriffe bei Nachweis einer morphologischen Schädigung oder Ausbleiben eines Therapieerfolges konservativer Verfahren eine oftmals nicht vermeidbare Therapieoption (Günther et al. 2011). Bei operativen Verfahren können 
dann zum Beispiel knöcherne Strukturen entfernt (z. B. Hemi-/Laminektomie), Segmente versteift (Fusion) oder der Versuch einer funktionserhaltenden Therapie (z. B. Implantation spezieller Bandscheibenprothesen) unternommen werden (Günther et al. 2011).

Die Segmentversteifung, auch Fusion genannt, stellt dabei bis heute den Goldstandard der verschiedenen Therapieverfahren dar (Kaner et al. 2010). Neben der Anwendung bei degenerativen Bandscheibenveränderungen findet es auch bei Diskusarthrose, segmentaler Instabilität oder Spondylolisthese Anwendung (Szpalski et al. 2002). Patienten berichten dann oftmals von kurzfristiger Schmerzfreiheit. Nach einiger Zeit stellt sich neben wiederkehrenden Schmerzen jedoch auch eine Überbelastung der umliegenden Segmente ein, was den Therapieerfolg nachhaltig beeinträchtigt. Dieses Phänomen wird auch adjacent segment disease (ASD) (Bertagnoli et al. 2006) oder adjacent level disease (ALD) genannt (Beutler et al. 2012). Die Segmentfusion kann also keineswegs hohen medizinischen Ansprüchen gerecht werden. Es ist daher zwingend erforderlich, Therapieverfahren zu entwickeln, die im Sinne der oben erwähnten funktionserhaltenden Maßnahmen versuchen, die physiologisch vorliegenden Verhältnisse nachzuempfinden und die Bewegungsmöglichkeit des Segmentes zu erhalten. Schon seit Jahrzehnten werden daher Versuche unternommen, physiologische Bandscheibenimplantate herzustellen (Szpalski et al. 2002). Die Entwicklung eines funktionserhaltenden Implantatsystems setzt dabei jedoch Kenntnisse über die in vivo vorliegenden biomechanischen Verhältnisse voraus. Diese Erkenntnisse zur Biomechanik der Wirbelsäule beschränkten sich lange Zeit lediglich auf rudimentäre anatomische und biomechanische Daten (Sanan und Rengachary 1996). Das Interesse an der klinischen Anwendung der Erkennt-nisse über die Biomechanik der Wirbelsäule gewinnt dabei jedoch schon über längere Zeit immer mehr an Bedeutung (Sanan und Rengachary 1996, Crawford 2006). Diese Entwicklung geht dahin, dass sich in jüngerer Zeit immer mehr Autoren um die Beschreibung der Segmentkinematik der Wirbelsäulensegmente bemühen. Dabei wurde erkannt, dass v.a. die Ermittlung einer Schraub-/Rotationsachse eine zuverlässige Analyse der Segmentkinematik zulässt (unter anderem: Haher et al. 1992, Kettler et al. 2004, Crawford 2006, Wachowski et al. 2009a, Wachowski et al. 2013).

Klinische Anwendung finden diese Kenntnisse in der Überlegung, dass sich die Lage der Schraub-/Rotationsachse nach Bandscheibenimplantation innerhalb des implantierten Segmentes im Vergleich zu gesunden Segmenten nicht unterscheiden sollte 
(Crawford 2006). Unter diesen Umständen können annähernd physiologische Bedingungen erhalten werden, was unter den derzeitigen Verfahren (Fusion der Segmente) ausgeschlossen ist.

Es ist beachtlich, dass trotz der hohen Prävalenz von Dorsopathien, dem hohen individuellen Leid des Patienten und der volkswirtschaftlichen Kosten derzeit keine adäquate Therapieoption, die die biomechanische Funktion der Wirbelsegmente wiederherstellen und somit eine dauerhafte Therapie bedeuten würde, Standard ist. Dies lässt die Frage aufkommen, ob es überhaupt einheitliche wissenschaftliche Erkenntnisse über die biomechanische Funktion, speziell Lage und Ausrichtung der Rotations-/Schraubachsen der Wirbelsäulensegmente gibt. Zwar befassen sich zahlreiche Studien mit dieser Thematik (z. B. Cossette et al. 1971, Panjabi et al. 1984, Kettler et al. 2004, Mansour et al. 2004 und andere), jedoch mangelt es bisher an einem Vergleich der Erkenntnisse der verschiedenen Autoren sowie einer Überprüfung der jeweils zur Ermittlung der Daten angewandten Methodik. Die vorliegende Arbeit soll daher die bisher fehlende Übersicht über die verschiedenen Untersuchungen liefern, die in der einschlägigen Literatur zu finden sind.

\section{Versuchsmethodiken der Studien}

Generell kann zwischen In-vivo-Versuchen und In-vitro-Versuchen sowie der Finiten Element-Methode (FEM) unterschieden werden. In-vivo-Versuche haben den Vorteil, dass von physiologischen Rahmenbedingungen ausgegangen werden kann (Smith 1991). Sie weisen jedoch im Vergleich zu In-vitro-Versuchen den Nachteil auf, dass die die Messergebnisse beeinflussenden Parameter weitestgehend unbekannt bleiben und wenig reproduzierbar sind (Smith 1991).

In der FEM werden die anatomischen Verhältnisse über eine computergestützte Simulation modelliert. Die virtuellen Versuchsbedingungen können dabei beliebig verändert und unter gleichen Bedingungen wiederholt werden. Jedoch sollte die Segmentkinematik erst an natürlichen Präparaten nachvollzogen werden, um sie in technisch aufwändigen computergestützten Verfahren näher untersuchen zu können.

In-vitro-Versuche weisen den Vorteil auf, dass die Versuchsbedingungen leicht validiert werden können (Spielmann 1994) und eine große Zahl an Präparaten untersucht werden kann (Panjabi et al. 1988). Jedoch ist dabei die Methodik, mit der die In-vitro-Versuche durchgeführt werden, von sehr hoher Relevanz, da nur unter be- 
stimmten Voraussetzungen physiologische Versuchsbedingungen erreicht werden und die Messresultate verwendet werden können (Smith 1991).

In der vorliegenden Analyse soll daher auf die Versuchsmethodiken und Messergebnisse von In-vitro-Untersuchungen eingegangen werden.

\section{Fragestellung der vorliegenden Arbeit}

Inwieweit wird eine momentane Schraub-/Rotationsachse in der Literatur zur Beschreibung der räumlichen Segmentkinematik der menschlichen Wirbelsäule untersucht?

In Kapitel 1.2 soll dazu zunächst auf den anatomischen Aufbau der Wirbelsäule eingegangen werden. Dabei sollen funktionelle Aspekte der Anatomie im Vordergrund stehen, auf anatomische Einzelheiten soll verzichtet werden. Einen besondereren Schwerpunkt bei der Einführung in die Thematik stellt dabei die Erklärung der Grundbegriffe der Biomechanik und Kinematik der Wirbelsäule dar (Kap. 1.3). Diese Grundlagen sollen im späteren Verlauf zur Analyse der vorgestellten Studien herangezogen werden. Im Methodikteil wird auf die Form der Literaturrecherche und die Methodik der Literaturauswahl eingegangen. Die durch die Literaturanalyse gefundenen Ergebnisse werden in Kapitel 3 vorgestellt. Dabei wird besonderes Augenmerk auf eine übersichtliche Zusammenstellung der verschiedenen Versuche gelegt. In Kapitel 4 werden die verschiedenen Methoden und Ergebnisse verglichen und bezüglich ihrer Aussagekraft über die Segmentkinematik untersucht.

\subsection{Funktionelle Anatomie der Wirbelsäule}

Die Wirbelsäule besteht aus 33 bis 34 Wirbeln. Diese werden nach anatomischer Lage in 7 Halswirbel (C1-C7), 12 Brustwirbel (Th1-Th12), 5 Lendenwirbel (L1-L5) sowie 5 Kreuzbein- und 4 bis 5 rudimentäre Steißbeinwirbel unterschieden (Schiebler und Korf 2007). Zusätzlich zu den zervikalen Wirbeln können auch die Kondylen des Occiputs als $\mathrm{C} 0$ bezeichnet werden. Die Anordnung der Wirbel ergibt im Sagittalschnitt eine doppelte S-Form (Aumüller et al. 2010). Bei einer nach dorsal konvexen Krümmung wird von einer Kyphose, bei einer ventralen Konvexität von einer Lordose gesprochen (Aumüller et al. 2010). Von kranial nach kaudal ergeben sich entsprechend die Bezeichnungen einer Zervikallordose, Thorakalkyphose, Lumballordose und Sakralkyphose. Funktionell kann die Wirbelsäule in frei bewegliche und nicht bewegliche Segmente unterteilt werden. Die miteinander 
verwachsenen Sakralwirbel und die rudimentären Kokzygealwirbel erlauben im Gegensatz zu den kranial gelegenen Wirbeln keine Bewegung (Voß 2011). Grundelemente der Wirbelsäule stellen die Wirbel (Vertebrae), Zwischenwirbelscheiben (Disci intervetebrales) und Bänder dar (Aumüller et al. 2010). Jeweils zwei aufeinander folgende Wirbel, die dazwischenliegende Bandscheibe und die verbindenden Bänder ergeben nach Junghanns ein Bewegungssegment (Junghanns 1977), auch functional spinal unit (FSU) genannt.

\section{Gelenke der Wirbelsäule}

Die Wirbelsäule besteht pro FSU aus drei gelenkigen Strukturen. Dazu gehören die paarigen Wirbelbogengelenke, auch Facettengelenke oder Articulationes zygapophysiales genannt und die gelenkige Verbindung zwischen den Bandscheiben, die durch die Deckplatten in Verbindung stehen. In der Halswirbelsäule gibt es zusätzlich noch die Unkovertebralgelenke. Diese Gelenke werden von den Procc. uncinati und Fissuren in der Bandscheibe gebildet und entstehen erst im Laufe des Lebens (Schünke 2000).

Die Bandscheiben bilden sogenannte Synarthrosen, durch ihre knorpeligen Deckplatten genauer auch Synchondrosen genannt. Die Synarthrosen sind keine echten Gelenke. Sie haben keinen Gelenkspalt und keine Gelenkkapsel. Die Bandscheibe stellt eine viskoelastische Scheibe dar, die der Bewegung 6 Freiheitsgrade ermöglicht (s.u.). Die Zwischenwirbelgelenke sind hingegen echte Gelenke und werden den Diarthrosen zugeordnet. Diarthrosen haben einen Gelenkspalt und eine Gelenkkapsel. Es ist bekannt, dass die Gelenke nicht formkongruent sind (Fick 1910).

\section{Wirbelkörper}

Die Wirbelkörper haben in ihrer Gesamtheit die Aufgabe das Rückenmark zu schützen, dem Körper Stabilität zu geben und erlauben durch ihre elastischen Verbindungen das Abfedern von Kräften, wie sie zum Beispiel bei Stoßkräften entstehen (Aumüller et al. 2010). Weiterhin bieten sie Ansatzstellen für zahlreiche Muskeln.

Zum Bauprinzip eines Wirbels gehört das Corpus vertebrae (Wirbelkörper), Arcus vertebrae (Wirbelbogen), das Foramen vertebrale (Wirbelloch) sowie die Wirbelbogenfortsätze (Aumüller et al. 2010). 
An allen Wirbelkörpern ist nach kranial bzw. kaudal ein Gelenkfortsatz erkennbar (Proc. articularis superior bzw. Proc. articularis inferior) (Platzer 2005). Die Gelenkfortsätze nehmen als sogenannte Diarthrosen (s. 1.4. Allgemeines zur biomechanischen Charakterisierung der Wirbelsegmentbewegung) entscheidenden Einfluss auf die Bewegung der Wirbelsäule (Platzer 2005).

Zusätzlich gibt es jedoch in den verschiedenen Abschnitten der Wirbelsäule einige anatomische Besonderheiten:

So finden sich bei den ersten beiden zervikalen Wirbelkörpern, dem Atlas (C1) und dem Axis (C2), Abweichungen. Der ringförmige Atlas besitzt keinen Wirbelkörper und artikuliert mit den superior gelegenen occipitalen Kondylen und dem inferior gelegenen Axis. Dieser ähnelt wiederum eher dem generellen Aufbau eines Wirbelkörpers, besitzt jedoch noch einen zusätzlichen knöchernen Vorsprung, den Proc. odontoideus oder Dens axis. Dieser artikuliert mit dem Atlas (Aumüller et al. 2010). An Körpern der Halswirbel finden sich zusätzlich noch die Procc. uncinati, auch Unci corporis genannt. Diese hakenförmigen Fortsätze richten sich im Laufe des Lebens auf und tragen so zur Bildung der Unkovertebralgelenke bei.

In der Brustwirbelsäule tragen die Procc. transversii Gelenkflächen (Fovea costalis transversalis), die sich mit dem Knochenhöcker (Tuberculum costae) der Rippen verbinden.

\section{Bandscheibe}

In die Boden- bzw. Deckplatte sowie die knöcherne Randleiste der Wirbelkörper inserieren Fasern des Anulus fibrosus (Aumüller et al. 2010). Der Faserring besteht aus einem konzentrisch verlaufenden Lamellensystem (Aumüller et al. 2010) und bildet zusammen mit dem Gallertkern (Nucleus pulposus) die Bandscheibe. Durch das hohe Wasserverbindungsvermögen der Glykosaminoglykane des Gallertkerns entsteht ein Quellungsdruck, der den Anulus fibrosus unter Spannung setzt (Platzer 2005). Durch den Quellungsdruck dienen die Bandscheiben als druckelastisches Polster (Platzer 2005). Bei Erwachsenen ist die Bandscheibe frei von Gefäßen. Die Ernährung erfolgt daher über Diffusion (Schiebler und Korf 2007). Bei Druckbelastung werden Wasser und Stoffwechselendprodukte aus der Bandscheibe gepresst. Bei abfallender Belastung wird die Bandscheibe wieder mit Wasser und 
Nährstoffen gefüllt. Während dieses Prozesses gewinnt bzw. verliert die Bandscheibe an Höhe. Die Bandscheibe hat somit eine wichtige mechanische Bedeutung.

\section{Bänder}

Die Bänder stabilisieren die Wirbelsäule und können zwischen einzelnen Wirbeln oder über größere Abschnitte verlaufen (Aumüller et al. 2010). Zudem haben sie eine wichtige Funktion bei Bewegungsvorgängen. Sie begrenzen das Bewegungsausma $B$ bei Extrempositionen und haben so großen Einfluss auf Biomechanik und Kinematik der Wirbelsäule. So begrenzt das Lig. longitudinale anterius mit den Ligg. flava die Dorsalextension, das Lig. Iongitudinale posterius, Lig. supraspinale, Lig. nuchae sowie die Ligg. interspinalia die Ventralflexion. Die Ligg. Intertransversaria begrenzen die Lateralflexion und Rotation (Aumüller et al. 2010).

\section{Muskeln}

Die Muskulatur des Rückens wird in eine tief gelegene autochthone Rückenmuskulatur und eine oberflächlich gelegene, nicht autochthone Rückenmuskulatur unterschieden (Voß 2011).

Durch die Fascia thoracolumbalis wird die autochthone Rückenmuskulatur an den Dorn und Querfortsätzen der Wirbel befestigt. Sie wird je nach topographischer Lage und Innervation in einen medialen und lateralen Trakt sowie den Trakt der kurzen Nackenmuskeln unterteilt (Aumüller et al. 2010). Die autochthone Muskulatur dient der Dorsalextension und Lateralflexion und wird von dorsalen Ästen der Spinalnerven innerviert (Aumüller et al. 2010). Da der mediale und laterale Trakt wesentlich an der Aufrichtung der Wirbelsäule beteiligt sind, werden sie auch als Musculus erector spinae bezeichnet und sind durch diese Funktion Antagonisten der seitlichen vorderen Bauchmuskulatur (Aumüller et al. 2010). Die ursprünglich aus ventralen Muskeln bestehende, eingewanderte, nicht autochthone Muskulatur wird im Unterschied zur autochthonen Muskulatur von ventralen Spinalnerven innerviert (Aumüller et al. 2010). Sie lässt sich in die spinokostalen Muskeln unterteilen, die an den Rippen ansetzen und an der Atemmechanik beteiligt sind, sowie die spinoskapulären Muskeln unterteilen, die am Schulterblatt ansetzen und an der Bewegung der oberen Extremität beteiligt sind (Aumüller et al. 2010).

Neben der Rückenmuskulatur übernimmt auch die ventrale Bauchmuskulatur eine wichtige Funktion bei der Bewegung des Rumpfes. Die Bauchmuskulatur lässt sich 
dabei in eine vordere, seitliche und hintere Muskelgruppe unterteilen (Aumüller et al. 2010). Sie beteiligen sich an der Drehung und Aufrichtung des Rumpfes (Aumüller et al. 2010). An der Ventralflexion ist wesentlich der Musculus rectus abdominis beteiligt, der daher auch als Hauptantagonist der Rückenmuskulatur bezeichnet wird (Aumüller et al. 2010). Ein sehr komplexes Zusammenspiel verschiedener Muskeln zeigt sich auch am Hals. Hier lassen sich die Muskeln in einen ventralen, medialen und lateralen Trakt einteilen. Hinzu kommen noch die kurzen Nackenmuskeln (Aumüller et al. 2010). Der mediale und laterale Trakt sowie die kurzen Nackenmuskeln übernehmen dabei hauptsächlich die Aufgabe der Extension und Drehung des Kopfes. Die ventrale Gruppe beteiligt sich neben der Drehung des Kopfes unter anderem auch an der Kieferöffnung (Aumüller et al. 2010). Auf die einzelnen Ursprünge und Ansätze der Muskeln soll an dieser Stelle nicht weiter eingegangen werden. Zusammenfassend lässt sich feststellen, dass der Rückenmuskulatur und Bauchmuskulatur neben der dynamischen Funktion auch eine stabilisierende Aufgabe zukommt (Aumüller et al. 2010).

\subsection{Physikalische Grundlagen räumlicher Bewegungsvorgänge starrer Körper}

\subsubsection{Kinematische Schraubung}

Zur Veranschaulichung wird der räumliche Bewegungsvorgang eines starren Körpers in Analogie zum Bewegungsvorgang eines Massenpunktes gesetzt.

Wenn ein Massenpunkt $m$ sich von einem Punkt A zu einem Punkt B bewegt, kann er dabei verschiedene Bewegungsbahnen zurücklegen. Abb. 1 zeigt drei mögliche Bewegungsbahnen. 
A

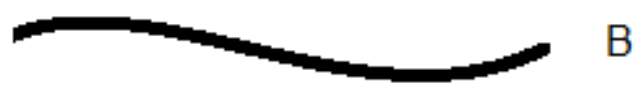

A

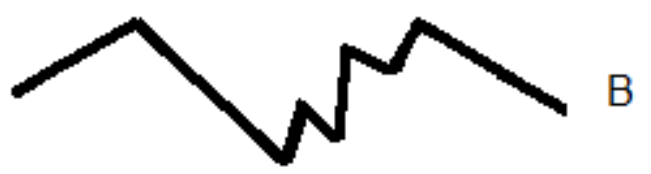

Abb. 1 Der Weg zwischen zwei Orten muss nicht entlang einer geraden Linie verlaufen, wenn auch Anfangs- und Endpunkt jeweils gleich sind.

Es sind unendlich viele Verlaufsbahnen denkbar. Wenn auch der Anfangs- und Endpunkt der Bewegung immer gleich ist, ist die zurückgelegte Strecke immer verschieden. Um die Bewegungsbahn nachvollziehen zu können, muss die Bewegungsbahn in differentiell kleine Segmente unterteilt werden.

Wie ein Massenpunkt, kann sich auch ein starrer Körper K im Raum von einer Lage A in die Lage B bewegen. Dabei kann er sowohl eine Translation als auch eine Rotation ausführen (Abb. 2). Eine solche Bewegung wird Schraubung genannt.
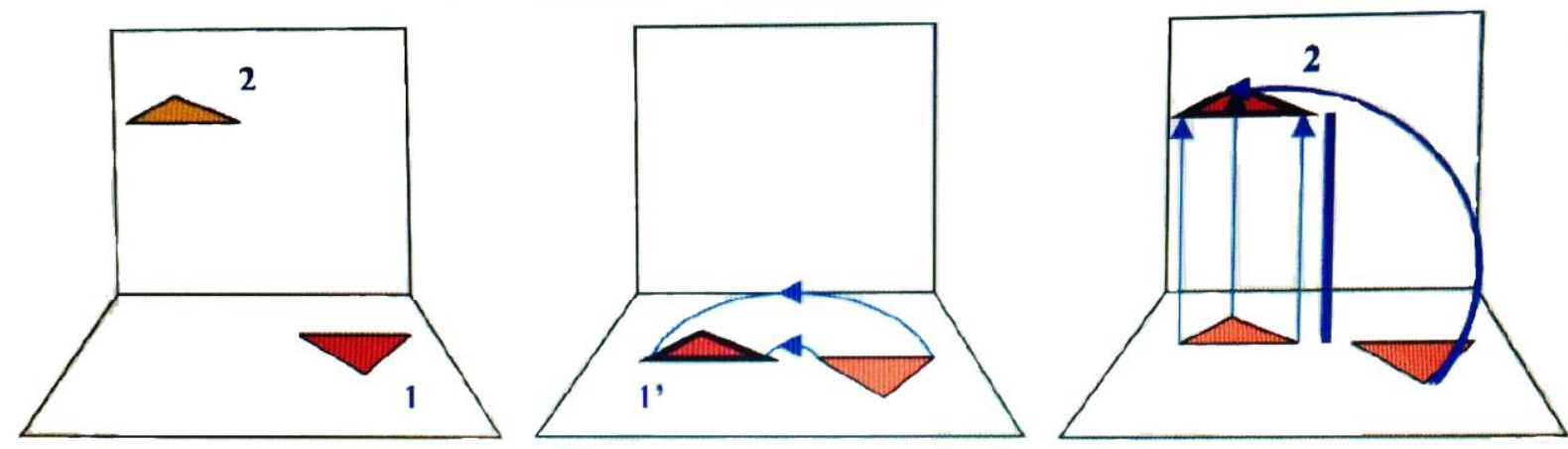

Abb. 2 Die Bewegung eines Körpers von der Position 1 in Position 2 kann in eine Rotationsbewegung (1') oder eine Translationsbewegung (2') unterteilt werden (Bockermann 2004, S. 20)

Diese Schraubung kann um eine ortsfeste Schraubachse ( $\mathrm{HA}=$ helical axis) erfolgen. Jeder Punkt des starren Körpers $K_{i}$ bewegt sich auf einer Schraubenlinie von seiner Ausgangslage $K_{i}(A)$ in seine Endpunktlage $K_{i}(B)$. Dabei muss die Schraubachse jedoch keineswegs ortsfest sein. Auch hier gilt, dass es unendlich viele Mög- 
lichkeiten der Überführung des Körpers $\mathrm{K}$ aus der Lage $\mathrm{A}$ in die Lage $\mathrm{B}$ gibt. Für jede Möglichkeiten ergeben sich wieder unterschiedliche Bewegungsstrecken. Bei Unterteilung der Bewegung in differentiell kleine Abschnitte, ergeben sich differentiell kleinen Schraubungen. Jede differentiell kleine Schraubung ist durch eine momentane Schraubachse (instantaneous helical axis= IHA) gekennzeichnet.

Die Gesamtheit der momentanen Schraubachsen ergibt eine Achsschar, aus der sich im ortsfesten Koordinatensystem die Regelfläche ergibt (Abb. 3).

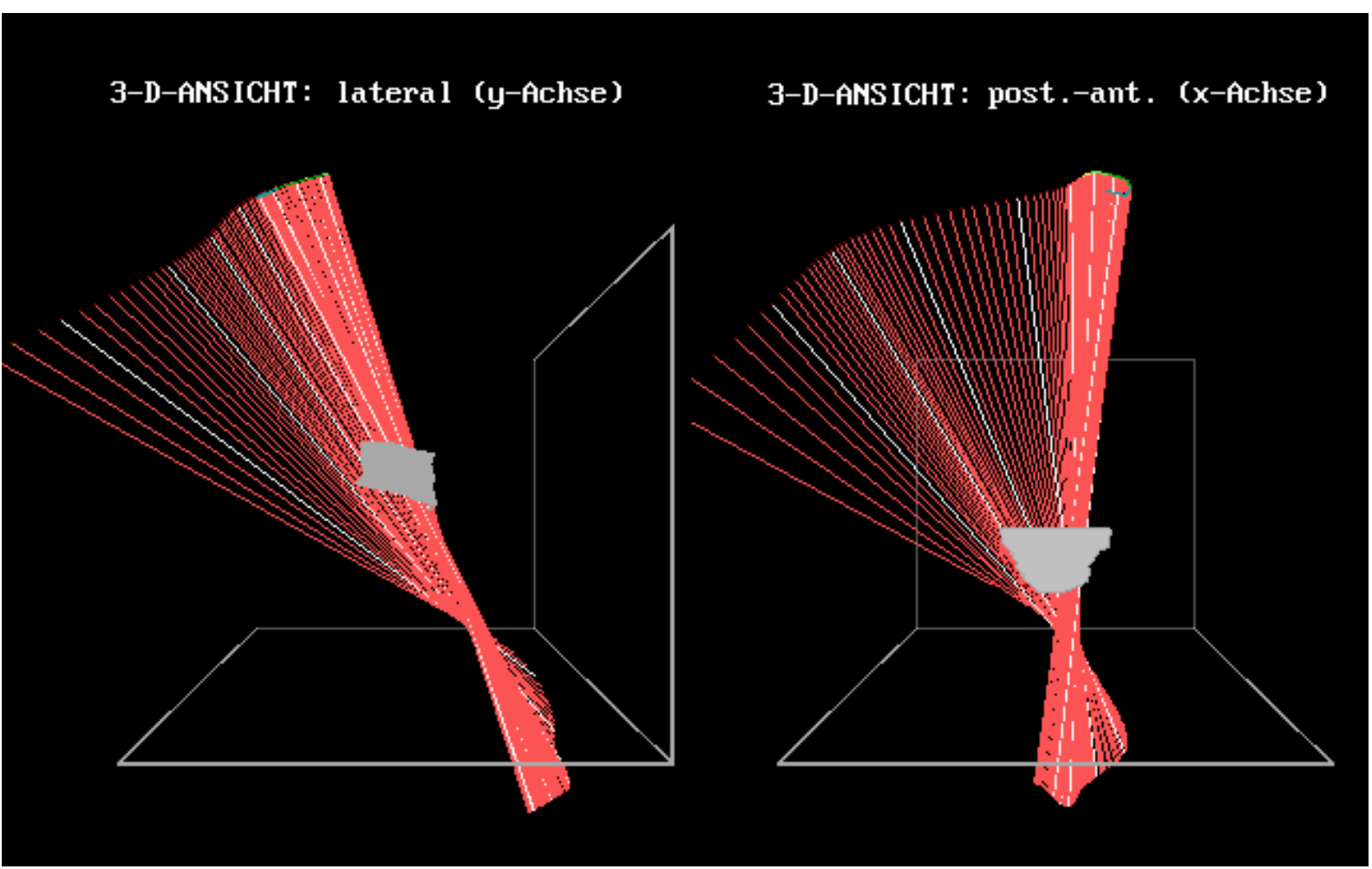

Abb. 3 Darstellung von Schraubachsen für die axiale Rotation in lateraler Ansicht und von hinten. Es zeigt sich eine Regelfläche, die sich aus der Achsschar ergibt (Bockermann 2004, S. 48).

Die momentane Bewegung eines Massenpunktes $m$ ist durch seine(n) Geschwindigkeit(svektor) $\vec{v}$ gegeben. Entsprechend der drei Komponenten des Geschwindigkeitsvektors hat ein Massenpunkt im Raum drei kinematische Freiheitsgrade (DOF = degree of freedom).

Zur Veranschaulichung: Der starre Körper kann in 3 Richtungen (entlang der x-, y-, zAchse) verschoben werden. D. h. er hat 3 translatorische Freiheitsgrade. Weiterhin kann sich der Körper bei der Bewegung um jede der drei Achsen des Koordinatensystems (x-, y-, z-Achse) drehen. Er hat also drei rotatorische Freiheitsgrade. Insgesamt ergeben sich also 6 Freiheitsgrade. 
Aus der momentanen Lage der IHA ergibt sich die momentane Bewegung eines starren Körpers K. Die momentane Lage ist durch den Abstandsvektor der Achse vom Koordinatenursprung und dem Richtungsvektor der Achse gegeben (Abb. 4). Die Lage der IHA hat 4 DOF.

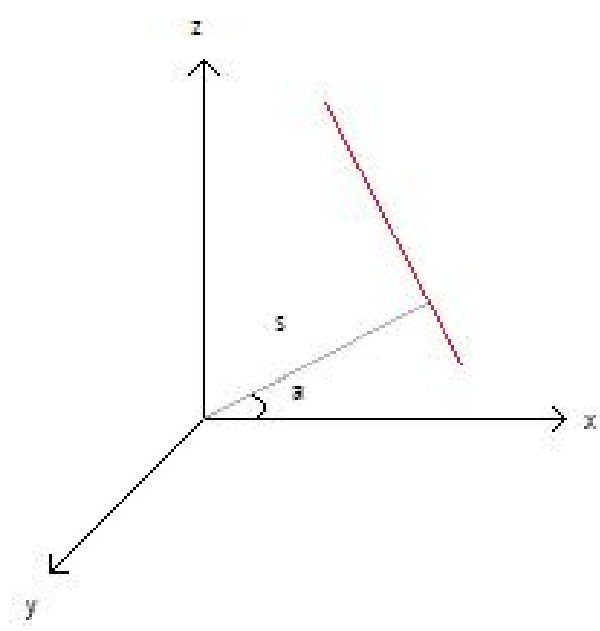

Abb. 4 Die momentane Lage einer Schraubachse ist durch den Abstandsvektor vom Koordinatenursprung und der Richtung gegeben, diese wird hier durch den Winkel a angezeigt. Die Lage der IHA hat 4 DOF.

Durch den Betrag der momentanen Winkelgeschwindigkeit $(\omega=d \alpha / d t)$ um die IHA (1 DOF) und durch den Betrag der momentanen Verschiebungs-Geschwindigkeit $\left(d s / d t=(d s / d \alpha) \cdot(d \alpha / d t)=s_{\alpha} \cdot \omega\right)$ entlang der IHA ergeben sich insgesamt 6 DOF. Dabei ist $s_{\alpha}$. die momentane Schraubsteigung der momentanen Schraubachse IHA. Die Schraubsteigung gibt den Versatz entlang der Schraubachse an, die durch die neben der Rotationsbewegung gegebenen Translationsbewegung entlang der Schraubachse definiert ist.

\subsubsection{Kraftschraube}

Jedes Kraftsystem, bestehend aus $\mathrm{n}$ Kräften $\vec{F}_{i}$ mit den Wirkungslinien $\vec{l}_{i}(1 \leq \mathrm{i} \leq \mathrm{n})$ und r Drehmomenten $\vec{T}_{j}(1 \leq \mathrm{j} \leq \mathrm{r})$ stellt eine Kraftschraube $\left(\vec{F}_{\text {res }}, \vec{T}_{\text {res }}\right)$ mit eindeutiger resultierender Wirkungslinie $\vec{L}_{r e s}$, der Kraftschraube IFS (instantaneous force screw = $\left(\vec{F}_{r e s}, \vec{T}_{r e s}\right)$ dar. Bezogen auf In-vitro-Versuche bedeutet dies, dass die Summe aller an ein Segment angreifender Muskelkräfte durch eine Kraftschraube simuliert werden kann. 


\subsection{Allgemeines zur biomechanischen Charakterisierung der Wirbelsegment- bewegung}

\subsubsection{Anatomie eines Wirbelsegmentes und daran orientierte anschaulich defi- nierte Begriffe}

Ein knöcherner Wirbelkörper wird mit einem kranial bzw. kaudal liegenden Wirbelkörper durch zwei Wirbelbogengelenke diarthrotisch bzw. durch verschiedene Bänder und jeweils einer Bandscheibe synarthrotisch verbunden (s. Kapitel 1.2 Funktionelle Anatomie der Wirbelsäule). Im Halsbereich finden sich mit den Unkovertebralgelenken noch zwei zusätzliche diarthrotische Gelenke. Bei einer ausschließlich synarthrotischen Verbindung hat die Lage des oberen Wirbels relativ zum unteren Wirbel sechs Freiheitsgrade (6 DOF). Durch Gelenkkräfte werden die Diarthrosen in Funktion gesetzt. Jede in Funktion gesetzte Diarthrose setzt die Freiheitsgrade des oberen Wirbels um 1 Freiheitsgrad herab.

Abbildung 5 stellt zwei Wirbelkörper in einem definierten Koordinatensystem mit einer möglichen Kraftschraube dar (Abb. 5).

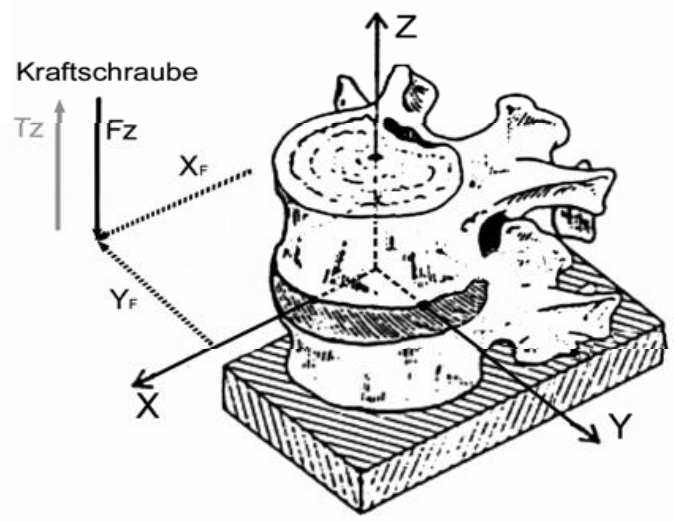

Abb. 5: Koordinatensystem und eine mögliche Kraftschraube bei axialer Rotation (Mansour 2001, S. 74)

Der Bewegung eines Wirbelkörpers liegen drei Bewegungsformen zugrunde, die sich aus der Symmetrie der Wirbelsegmente ergeben: Flexion/Extension, Lateralflexion und Axialrotation.

Drehmomente, die senkrecht zur Axialebene gerichtet sind, verursachen axiale Rotation. Die entsprechenden momentanen Schraubachsen (IHA) sind dann etwa parallel 
zum angelegten Drehmoment. Entsprechendes gilt für Lateralflexion und FlexionExtension.

Prinzipiell handelt es sich bei der Bewegung der Wirbelkörper jedoch nicht um eine rein plane Bewegung. Vielmehr liegt, bedingt durch die Inkongruenz der Gelenkflächen eine dreidimensionale gekoppelte Bewegung (engl. coupled motion) vor (Wilke et al. 1998a).

\subsection{Grundlagen der In-vitro-Untersuchung der Wirbelsegmentbewegung}

Zwei Aspekte sind beim Planen eines experimentellen Aufbaus zu beachten: a) Wie wird die Bewegung der Wirbelsegmente experimentell erzeugt? b) Welche Bewegungsstruktur zeigt ein Wirbelkörper innerhalb eines Segmentes in Bezug zum anderen Wirbelkörper nach Anlegen eines zeitlich veränderlichen Kraftsystems?

\subsubsection{Wahl des angreifenden Kraftsystems}

Hierzu sind wiederum zwei Kriterien für die experimentelle Realisierung entscheidend: 1.) Wo wird und 2.) wie kann das Kraftsystem rückwirkungsfrei auf den bewegten Wirbel übertragen werden?

Das Drehmoment ist im Gegensatz zur Kraft ein freier Vektor und besitzt daher keine Wirkungslinie. Wo das Drehmoment auf einen starren Körper (knöcherner Wirbel) übertragen wird, ist daher nicht relevant. Die resultierende Bewegung ist in jedem Fall gleich. Wie das Kraftsystem möglichst rückwirkungsfrei auf den Wirbel übertragen werden kann, wird hingegen vom Aufbau einer Testapparatur bestimmt, was in der vorliegenden Arbeit zu untersuchen ist.

\subsubsection{Voraussetzungen zur Messung der Segmentbewegung}

Um einen Einfluss der Testapparatur auf die Bewegung des Wirbelsegmentes auszuschließen, muss dieser sich in allen 6 Freiheitsgraden bewegen können. Zudem muss die Wirbelbewegung in allen 6 Freiheitsgraden rückwirkungsfrei gemessen werden. Messungen, die weniger als 6 Freiheitsgrade erlauben, sind nicht valide, da dann die Bewegungsstruktur durch den Aufbau der Testapparatur beeinflusst wird. 
Nach Gregersen und Lucas (1967) beträgt die maximale physiologische axiale Rotation im Lendenbereich etwa $\pm 1,5^{\circ}$. Es handelt sich also nur um kleine Bewegungen. Eine Schraubachse, die aus zwei Positionen berechnet wird, die sich beispielsweise um $2^{\circ}$ unterscheiden ist daher nicht valide. Es ist offensichtlich, dass dieser Bewegungsbereich zu groß ist, um eine valide Beschreibung der Bewegungsstruktur angeben zu können. Die Schraubachse wäre somit annähernd ortsfest. Um die Wanderung der IHA aufzulösen und damit die tatsächliche Bewegung zu registrieren, muss Folgendes gelten:

$$
\operatorname{IHA}(\alpha)=\lim _{\Delta \alpha \rightarrow 0} F H A_{\alpha}^{\alpha+\Delta \alpha}
$$

Es muss daher eine $\mathrm{FHA}\left(\alpha_{\mathrm{i}}\right)$ bestimmt werden, deren Winkelabstand $\Delta \alpha$ möglichst klein gegenüber dem physiologischen Maximum von $\pm 1,5^{\circ}$, also $\Delta \alpha<0.03^{\circ}$ ist. Die angewandte Messtechnik zur Auflösung der Bewegung muss daher mindestens 1 $\mu \mathrm{m}$ für Translationen und $0,001^{\circ}$ für Rotationen entsprechen (Nägerl 2014 persönliche Mitteilung).

\subsubsection{Kennlinien der Segmente}

Bei angelegter periodischer Zeiffunktion des Drehmoments $(T(t))$ und gemessener periodischer Drehwinkel-Zeitfunktion $(\alpha(t))$ ergeben sich DrehwinkelDrehmomentkennlinien $(\alpha=\alpha(T))$.

Die Kennlinie ist nichtlinear und zeigt eine Hysterese (Abb. 6). Das Ausmaß der Hysterese hängt unter anderem von der Frequenz der periodischen Zeitfunktion des Drehmoments ab (Nägerl 2014 persönliche Mitteilung). 


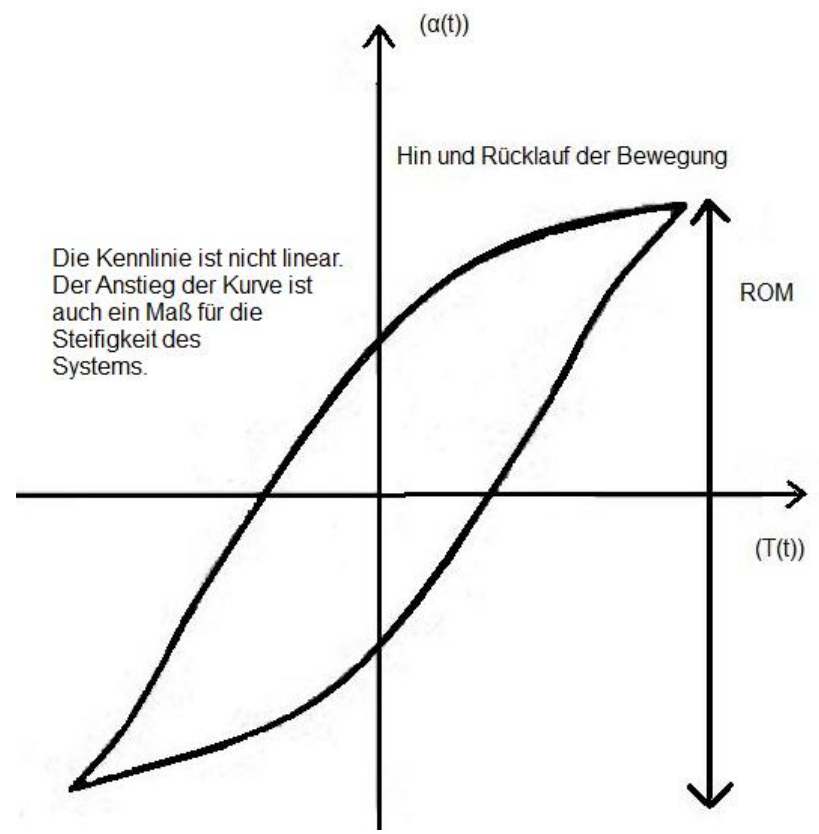

Abb. 6 Möglicher Verlauf einer Bewegung. Die Aufzeichnung des Drehwinkels in Abängigkteit vom zyklischen Prozess der Drehmomentapplikation. Die von den Kennlinien der Hin- und Rückbewegung umschlossene Fläche wird als Hysteresefläche bezeichnet.

Diese Kennlinien werden in der Literatur weiter in das Bewegungsausmaß range of motion (ROM) und die neutralen Zone (NZ den linear verlaufenden Teil bei kleinen Drehmomenten) (Panjabi 1992) unterteilt. Physikalisch handelt es sich jedoch nicht um kinematische Begriffe (Nägerl 2014 persönliche Mitteilung). Denn bei größerem Drehmoment, ist auch das Bewegungsausmaß größer und die empirisch abgelesene NZ enthält keine Aussagekraft über die Kinematik der Segmentbewegung. Für diesen Bereich gilt lediglich, dass der Differentialquotient $\mathrm{da} / \mathrm{dT}=$ konstant ist (Nägerl 2014 persönliche Mitteilung). Der Bereich nach der NZ bis zum Erreichen des vollen ROM wird elastische Zone (EZ) genannt. Der EZ folgt die plastische Zone (PZ), in der das Präparat irreversibel geschädigt wird, was erreicht wird, wenn das Drehmoment hinreichend groß ist (Abb. 7). 


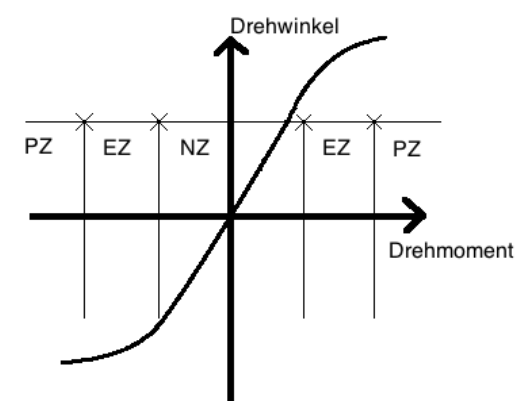

Abb. 7 Darstellung einer Drehwinkel- Drehmomentkurve mit Einteilung des ROM in NZ, EZ und PZ ( $\mathrm{NZ}=$ neutrale Zone, $\mathrm{EZ}=$ elastische Zone, $\mathrm{PZ}=$ plastische $Z$ Zone)

Physikalische Hintergründe der Kennlinien $(\alpha=\alpha(T))$ : Ohne den Einfluss von Bändern und Gelenken verhält sich die Bandscheibe wie eine viskoelastische Scheibe mit ortsfester Drehachse.

Innerhalb der Kennlinie ist der Bereich mit Differentialquotient da/dT = konstant viel größer als beim intakten Segment. Bei großen Drehmomenten wird dann die Kennlinie auch nichtlinear, was mit der Nichtlinearität des Materials zusammenhängt. Bei intakten Segement sind die Verhältnisse komplizierter. Je nach Verformung sind die die Bänder in Funktion gesetzt und tragen so zur Steifigkeit der Bandscheibe bei. Wird die Bewegung des Segmentes auch durch die diarthrotischen Gelenke geführt, so werden die Verhältnisse noch umfangreicher. Die Inkongruenz der Gelenkoberfächen hat nämlich entscheidenden Einfluss auf die IHA. Dies betrifft die Lage und Richtung sowie die Schraubsteigung (s. Kap. 1.3.1), was auch die Steigung da/dT beeinflusst und zur Nichtlinearität der Kennlinie beiträgt.

Schlussfolgerung: Das Messen kinematischer Größen (Wanderung und Ausrichtung der momentanen IHA) ist für das Erfassen der Segmentbewegung unerlässlich.

\subsubsection{Im Schrifttum verwendete Begriffe zur Beschreibung der Rotations-/ Schraubachse}

In der Literatur wird bezüglich der Schraubachse zwischen einer helical axis of motion (HAM) (Cripton et al. 2001, Zhu et al. 2007, Takigawa et al. 2010), finite helical axis of motion (FHA) (Kettler et al. 2004), instantaneous axis of rotation (IAR) (Haher et al. 1992), axis of rotation (Detwiler et al. 2003), rotational axis (Molnar et al. 2006), helical axis oder instantaneous helical axis (IHA) (Mansour et al. 2004) unterschieden. Diese terminologischen Abgrenzungen sind jedoch physikalisch nicht begründet. 
Es gilt in der vorliegenden Arbeit die Bedeutung dieser unterschiedlichen Begrifflichkeiten zu beleuchten und ihre Unterschiede an den unter Kapitel 1.6 genannten Forderungen herauszuarbeiten. Alle Achsenarten haben gemeinsam, dass sie eine dreidimensionale Struktur darstellen, um die die Rotationen und Translationen erfolgen. Als wichtige Voraussetzung muss gelten, dass die Lage der ermittelten Rotations-/Schraubachse zu jedem Zeitpunkt der Bewegung bekannt ist. Das heißt, dass die Bewegungsintervalle zwischen den Messungen differentiell klein sein müssen, da sonstige Lagemessungen Mittelwerte darstellen und nicht valide sind (Nägerl 2014 persönliche Mitteilung).

\subsection{Kriterien zur Beurteilung der in der vorliegenden Arbeit zu untersuchenden Studien:}

Anhand der genannten anatomischen und biomechanischen (kinematischen) Gegebenheiten sollen im Folgenden die zur Beurteilung der zu untersuchenden Studien notwendigen Kriterien formuliert werden.

Aus den genannten geometrischen und anatomischen Gegebenheiten (s. Kap. 1.21.5) lassen sich entsprechend Forderungen für die Umsetzung von In-vitroUntersuchungen der Wirbelsäule formulieren, an denen die zu untersuchenden Studien gemessen werden sollen:

1. Während des Testdurchlaufes müssen die zu untersuchenden Testobjekte durch eine Testapparatur in Bewegung gesetzt werden. Diese Apparatur darf jedoch die Bewegungsstruktur der Testobjekte nicht beeinflussen (6DOF). D. h. die Bewegung der Segmente muss in Bezug auf die Testapparatur rückwirkungsfrei in allen möglichen Bewegungsrichtungen erfolgen können.

In Kapitel 3.2 wird daher auf die verschiedenen Testapparaturen und Formen der Drehmomentapplikation der zu untersuchenden Studien eingegangen.

2. Die an den Segmenten ansetzenden Muskelkräfte und Gewichte müssen durch eine Kraftschraube realisiert werden. 
In Kapitel 3.2 sollen daher die Formen der Applikation des Drehmomentes und in Kapitel 3.3 die Formen der Applikation einer „axialen Vorlast“ dargestellt werden, aus denen sich die resultierende Kraftschraube ergibt.

3. Es sollte eine möglichst große Zahl an Präparaten verwendet werden, die keinen pathologischen Zustand aufweisen, um möglichst allgemeingültige Testergebnisse erhalten zu können.

In Kapitel 3.4 wird auf die in den zu untersuchenden Studien verwendeten Präparate eingegangen.

4. Um den Bewegungsvorgang eines Körpers zu erfassen, müssen multiple Lagen des Körpers bekannt sein, die von differentiell kleinen Bewegungsintervallen getrennt sind. Es gilt daher die Forderung: $\Delta \alpha<<R O M$.

Ist das gemessene Bewegungsintervall nicht differentiell klein, ist der Bewegungscharakter unbekannt (vgl. Kapitel 1.3.1). Angaben zur Bewegungsstruktur wären in diesen Fällen daher nicht valide. In Kapitel 3.5 werden daher die verschiedenen Messapparaturen hinsichtlich ihrer örtlichen und zeitlichen Auflösungsfähigkeit verglichen.

Des Weiteren wird in Kapitel 3.6 nach Analyse der zu untersuchenden Studien, erarbeitet, inwiefern eine einheitliche Terminologie bezüglich der kinematischen Beschreibung der Bewegungsstruktur der Wirbelsäule besteht.

In Kapitel 3.7 wird ein konkreter Vergleich der Ergebnisse der untersuchten Studien in Bezug auf die Lagemessungen der Rotations-/Schraubachsen angestellt. Dabei soll v. a. auf Untersuchungen mit zervikalen und lumbalen Segmenten eingegangen werden.

In Kapitel 3.8 werden Angaben der Autoren bezüglich weiterer häufig verwendeter biomechanischer Parameter wiedergegeben. 


\section{Methodik}

\section{Verwendete Datenbanken und Suchbefehle}

Es wurde auf die Datenbanken PUBMED und WEB OF SCIENCE zurückgegriffen. PUBMED gehört zu den bekanntesten medizinischen Datenbanken, bietet zusätzlich Zugriff auf die Datenbank MEDLINE und verzeichnet über 5600 Zeitschriften aus dem Bereich Biomedizin.

Das WEB OF SCIENCE wertet neben dem medizinischen Fachbereich auch Zeitschriften aus dem Gebiet der Naturwissenschaften aus. Weiterhin kann über die Erstellung eines citation reports auf zitierte und die jeweils vorliegende Studie zitierende Studien zurückgegriffen werden. Dieses Verfahren wurde für alle untersuchten Studien angewandt. Eine Literaturrecherche in der Datenbank COCHRANE LIBRARY lieferte keine Suchergebnisse, da bei dieser Datenbank Literatur über medizinische Behandlungsmethoden im Mittelpunkt steht. Die Datenbank MEDPILOT zeigte eine für die vorliegende Arbeit nicht angemessene Suchfunktion, da hier nicht genügend Begriffe kombiniert werden können. Sie wurde daher nicht berücksichtigt. Die Recherche erfolgte in den genannten Datenbanken ausschließlich mit englischen Suchbegriffen.

Folgende Suchbegriffe wurden festgelegt:

-„axis of rotation“ spine

-„axes of rotation" spine

-,finite helical axis“ spine

-„,helical axis” spine

-„,helical axis of motion” spine

-,instantaneous helical axis” spine

-,instantaneous axis of rotation” spine

- kinematic testing in vitro spine

Nach Kombination der Suchbegriffe mit Hilfe der Suchfunktion advanced search ergab sich folgende Suchsyntax für PUBMED: 
((()(("axis of rotation"[All Fields] OR (axes[All Fields] AND ("rotation"[MeSH Terms] OR "rotation"[All Fields]))) OR (helical[All Fields] AND ("axis"[MeSH Terms] OR "axis"[All Fields]) AND ("motion"[MeSH Terms] OR "motion"[All Fields]))) OR "finite helical axis"[All Fields]) OR "instantaneous helical axis"[All Fields]) OR (instantaneous[All Fields] AND ("axis"[MeSH Terms] OR "axis"[All Fields]) AND ("rotation"[MeSH Terms] OR "rotation"[All Fields]))) OR "helical axis"[All Fields]) OR (("biomechanical phenomena"[MeSH Terms] OR ("biomechanical"[All Fields] AND "phenomena"[All Fields]) OR "biomechanical phenomena"[All Fields] OR "kinematic"[All Fields]) AND testing[All Fields] AND ("in vitro"[Publication Type] OR "in vitro"[All Fields]))) AND ("spine"[MeSH Terms] OR "spine"[All Fields])

Für die gleichen Suchbefehle (s.o.) ergab das WEB OF SCIENCE folgende Suchsyntax:

("axis of rotation") OR TOPIC: ("axes of rotation") OR TOPIC: ("helical axis") $O R$ TOPIC: ("helical axis of motion") OR TOPIC: ("finite helical axis") OR TOPIC: ("instantaneous helical axis") OR TOPIC: ("instantaneous axis of rotation") OR TOPIC: (kinematic testing in vitro) $A N D$ TOPIC: (spine)

Im WEB OF SCIENCE wurde auch die Option citation report genutzt. Für jede der gefundenen Studien wurden alle zitierten und alle Studien, die die jeweilige Studie zitieren, ausgewertet und gegebenenfalls (bei Erfüllung der genannten Kriterien) zur Analyse herangezogen.

\section{Ein- und Ausschlusskriterien:}

Unter den gefundenen Artikeln wurden gemäß der gesetzten Zielstellung der vorliegenden Studie die Artikel ausgewählt, die folgende Einschlusskriterien erfüllten:

- In der Studie werden als Messparameter Angaben zu einer Rotations-/ Schraubachse der Wirbelsäulensegmente ermittelt

- Es handelt sich um einen In-vitro-Versuch

- In der Studie werden Angaben zum Versuchsverfahren gemacht 
Von der Untersuchung wurden ausgeschlossen:

- In-vivo-Untersuchungen

- Studien mit Anwendung der Finite-Element-Methoden (FEM)

- Studien, in denen keine Rotations-/Schraubachsen der Wirbelsäule ermittelt wurden

- Habilitations- oder Dissertationsschriften

- Veröffentlichungen von Kongressvorträgen

Eine erste Vorauswahl der Studien erfolgte durch Lesen des Abstracts der gefundenen Studien. Eine weitere Auswahl erfolgte durch Sichtung der gesamten Veröffentlichung. 42 Studien erfüllten die Suchkriterien.

Die zur Untersuchung herangezogenen Artikel wurden durch direkte Suche in den jeweiligen Journals über die elektronische Zeitschriftenbibliothek (EZB) der GeorgAugust-Universität Göttingen abgerufen. Artikel, für die keine Lizenzen vorlagen, wurden über die Fernleihe der Staats- und Universitätsbibliothek Göttingen bestellt. In einigen Fällen mussten zum Verständnis eines Versuches weitere Studien herangezogen werden, auf die in dem jeweiligen Artikel Bezug genommen wurde. In manchen Fällen erfolgten unvollständige oder unzureichende Angaben bezüglich der Versuchsmethodik, was eine valide Evaluation der Messergebnisse hinsichtlich der Zielstellung der vorliegenden Arbeit erschwerte. In diesen Fällen wurden durch direktes Anschreiben der Autoren Zusatzinformationen eingeholt. Dabei handelte es sich im Wesentlichen um Angaben zur jeweiligen verwendeten Messapparatur (s. Kap. 3.5). Der Schriftverkehr wurde archiviert und ist auf Nachfrage beim Autor der vorliegenden Studie einsehbar. Die zusätzlich herangezogenen Informationen sind zudem im Literaturverzeichnis vermerkt (Kap. 6). 


\section{Testverfahren/ Messergebnisse}

\subsection{Zielstellungen der untersuchten Studien}

Die im Rahmen der Literaturrecherche gefundenen Studien wurden zunächst systematisch in Untergruppen eingeteilt. In einigen Fällen fanden sich die Studien in mehreren Untergruppen wieder.

Erstens fanden sich Studien, die das biomechanische und kinematische Verhalten der Wirbelsäule direkt in den Mittelpunkt ihrer Untersuchung stellten (Haher et al. 1991, Haher et al. 1992, Grassmann et al. 1998, Cripton et al. 2001, Crawford et al. 2002, Haberl et al. 2004, Kettler et al. 2004, Mansour et al. 2004, Molnar et al. 2006, Rousseau et al. 2006a, Wachowski et al. 2007, Nägerl et al. 2009, Wachowski et al. 2009a, Wachowski et al. 2009b, Dugailly et al. 2010, Wachowski et al. 2010a, Wachowski et al. 2010b, Dugailly et al. 2011, Sengupta et al. 2011, Schulze et al. 2012, Dugailly et al. 2013).

Zweitens: In einigen Fällen wurden biomechanische und kinematische Parameter genutzt, um Veränderungen nach chirurgischen Eingriffen wie Implantation von Bandscheibenprothesen, (Kettler et al. 2004, Rousseau et al. 2006b, Takigawa et al. 2010, McNally et al. 2012, Zhu et al. 2012, Wachowski et al. 2013), Stabilisierungsverfahren (Cusick et al. 1988, Dickman et al. 1996, Naderi et al. 1998, Cagli et al. 2004, Niosi et al. 2006, Zhu et al. 2007, Bozkus et al. 2010, Lazaro et al. 2010, Zhu et al. 2012) oder Verletzungen bzw. Resektion von anatomischen Strukturen der Bewegungssegmente zu untersuchen (Cusick et al. 1988, Haher et al. 1991, Haher et al. 1992, Dickman et al. 1996, Broc et al. 1997, Oxland et al. 1994, Chen et al. 2001, Crawford et al. 2002, Detwiler et al. 2003, Cagli et al. 2004, Kettler et al. 2004, Wachowski et al. 2007, Bozkus et al. 2010, Wachowski et al. 2010b, Brasiliense et al. 2011).

In der dritten Untergruppe wurden kinematische und biomechanische Veränderungen nach degenerativen Prozessen der zu testenden Wirbelsäulensegmente untersucht (Seligman et

al. 1984). 


\subsection{Testapparatur und Drehmomentapplikation}

\subsubsection{Testapparatur}

Die Autoren verwendeten unterschiedliche Testapparaturen. Zum Grundaufbau einer Testapparatur gehörte unter anderem ein fester Rahmen, der aus Stahl (z. B. Mansour et al. 2004, Wachowski et al. 2013 und andere), Aluminium (z. B. Niosi et al. 2006, Zhu et al. 2007 und andere) oder Kunststoff (z. B. Haher et al. 1992 und andere) bestand und in dem das Präparat am oberen und unteren Ende fest eingespannt war. Abbildungen 8 und 9 (S. 23, S. 24) zeigen dabei exemplarisch häufig verwendete Testapparaturen.

\subsubsection{Drehmomentapplikation}

Bei der Drehmomentapplikation unterschieden sich die Studien in Bezug auf die Richtung des angelegten Drehmomentes, die resultierende Drehgeschwindigkeit des Präparates und die angewandte Drehmomentgröße (Tab. 1). Bei der resultierenden Rotationsgeschwindigkeit der Präparate fanden sich Werte von $0,5 \% \mathrm{~s}$ (Zhu et al. 2012) bis zu $10 \%$ s (Naderi et al. 1998). Um, laut den Autoren, viskoelastischen Effekten der Bandscheibe Rechnung zu tragen und Reibungswiderstände zu reduzieren, gab es zwischen den Testdurchläufen mit verschiedenen Drehmomentgrößen oftmals eine Unterbrechung (Tab. 1). Die Anzahl der ermöglichten Freiheitsgrade der Bewegung (Tab. 1) wurde dabei durch den Aufbau der Testapparatur und die Art der Drehmomentapplikation bestimmt.

Die Applikation des Drehmomentes erfolgte in allen untersuchten Studien auf den in der Testapparatur obersten Wirbel bzw. an den Befestigungsbehälter des obersten Wirbels.

Um ein Drehmoment zu erzeugen, verwendeten die Autoren servohydraulisch, pneumatisch bzw. Wasserpumpen betriebene Generatoren sowie elektrisch betriebene Motoren (s.u.). In einigen Fällen wurde das Drehmoment manuell durch den Untersucher oder durch Gewichte erzeugt (s.u.). Weiterhin wählten die Autoren verschiedene Übertragungselemente, die genutzt wurden, um das vom Generator/Motor induzierte Drehmoment auf das Präparat zu übertragen:

Haberl et al. (2004) applizierten in einem Versuch axiale Drehmomente über eine horizontal ausgerichtete Umlenkrolle, die über dem obersten Wirbel angebracht war. Angaben zum verwendeten Drehmomentgenerator erfolgten nicht. Zusätzlich wurden über dem Segment befestigte, seitlich des Segmentes herabhängende, nach anterior 
und posterior verschiebbare Gewichte zur Applikation von Flexions- und Extensionsmomenten angebracht. Zugleich wurde dadurch eine axiale Vorlast appliziert (s. Kap. 3.3) (Haberl et al. 2004). Ein ähnliches Prinzip aus Kombination von axialer Vorlast (s. Kap. 3.3) und Flexions- und Extensionsbewegungen verwendeten auch Bozkus et al. (2010), und Lazaro et al. (2010). Dabei wurden allerdings keine generierten Drehmomentgrößen angegeben (s. Tab. 1). Die Drehmomente wurden soweit erhöht, bis ein bestimmtes, zuvor definiertes Bewegungsausmaß erreicht wurde. Die Autoren verwendeten in ihren Versuchen eine weitere Apparatur, mit der rückwirkungsfreie Bewegungen in allen drei Ebenen möglich gewesen seien (Bozkus et al. 2010, Lazaro et al. 2010). Diese von Crawford et al. vorgestellte Apparatur (s. Abb. 8), wurde später von zahlreichen weiteren Autoren für die Testdurchläufe verwendet und soll daher näher beschrieben werden (s.u.). Mit der verwendeten Apparatur sollten nahezu rückwirkungsfreie Drehmomente möglich sein (Dickman et al. 1996, Broc et al. 1997, Naderi et al. 1998, Chen et al. 2001, Crawford et al. 2002, Detwiler et al. 2003, Cagli et al. 2004, Lazaro et al. 2010, Brasiliense et al. 2011, Bozkus et al.2010). Die Apparatur applizierte Drehmomente in allen drei Bewegungsrichtungen über einen servohydraulischen Antrieb. Dieser befand sich im unteren Teil der Apparatur und generierte durch Abwärtsbewegung eine Spannung in Kabeln, die wiederum über Umlenkrollen den obersten Wirbel in Bewegung versetzten. Wichtige Voraussetzung war dabei, dass die Kabel nachdem sie die Umlenkrollen umlaufen haben stets parallel zueinander und in der Horizontalebene verliefen, was durch manuelle Einstellung der Untersucher eingestellt werden sollte. 


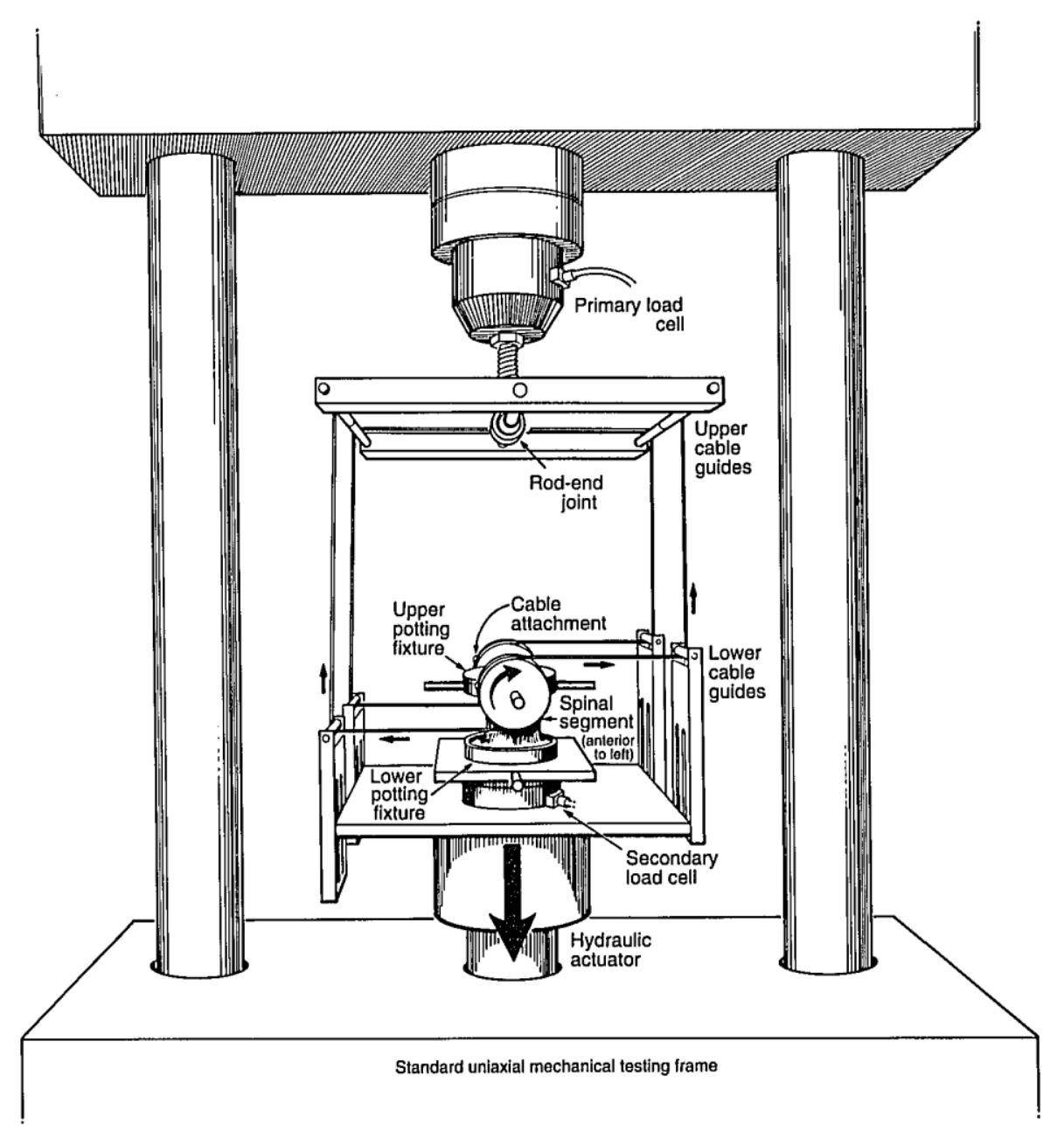

Abb. 8: Häufig verwendete Apparatur (Crawford et al. 1995, S. 2098; mit Erlaubnis von Lipincott Williams and Wilkins/ Wolters Kluwer Health).

Ein anderer Apparaturaufbau zeigte sich bei McNally et al. (2012). Die Autoren applizierten Flexionsmoment und Vorlast (s. Abb. 10 S. 98), indem sie eine kompressive Last auf das Präparat anlegten, die über eine Rolle in der Mittsagittalen nach anterior verschiebbar war (s. Kap. 3.3). Der Aufbau der Testapparatur unterband gezielt Bewegungen außerhalb der getesteten Ebene (McNally et al. 2012). Ein ähnliches Prinzip nutzten auch Cusick et al. (1988).

Rousseau et al. und Haher et al. generierten keine kontinuierlichen Bewegungen sondern gaben die Stellung über axiale Kompressionskräfte und Keile vor. Diese verursachten dann eine Stellungsveränderung des Segmentes im Sinne einer Flexion bzw. Extension (Haher et al. 1991, Rousseau et al. 2006a, Rousseau et al. 2006b). 
Grassmann et al. (1998) testeten zwei Apparaturen, um den Unterschied zwischen einer Apparatur, in der die Bewegung durch die Testapparatur und Drehmomentapplikation vorgegeben wird (constrained) und einer Testatapparatur, in der sich das Segment unabhängig von Testapparatur und der Art der Drehmomentapplikation (unconstrained) bewegt, zu untersuchen. Das Drehmoment in der zuletzt genannten Apparatur wurde durch pneumatische Zylinder generiert und über Kabel und Umlenkrollen, die an der Fixierung des oberen Wirbels befestigt waren, auf das Segment übertragen. Das obere Segment wurde nicht von weiteren Elementen der Testapparatur beeinflusst und konnte sich so nach Angaben der Autoren frei bewegen. In der constrained Testapparatur erfolgte die Bewegung um zuvor definierte Rotationsachsen. Bei Versuchen mit dieser Testapparatur wurden also keine Rotations-/Schraubachsen ermittelt (Grassmann et al. 1998). Sie wird daher nicht weiter beschrieben.

Andere Autoren applizierten das Drehmoment, indem sie mit Wasser gefüllte Behälter über Seile an das obere Segment anbrachten und über ein Pumpsystem einen kontinuierlichen Wasserfluss zwischen den Behältern generierten (Mansour et al. 2004, Nägerl et al. 2009, Wachowski et al. 2007, Wachowski et al. 2009a, Wachowski et al. 2009b, Wachowski et al. 2010, Wachowski et al. 2010b, Wachowski et al. 2013, Takigawa et al. 2010) (s. Abb. 9). Durch das sich ändernde Gewicht der Behälter wirkten entsprechend Drehmomente, die wiederum Bewegung generierten. Einige Autoren verwendeten dabei vier an einem Stahlkreuz (Kraftkreuz) befestigte Behälter. Zwischen zwei Behältern erfolgte der Wasserfluss, die anderen zwei dienten als Kompensation. Das Gewicht des Kraftkreuzes wurde ebenfalls durch ein Gegengewicht kompensiert (Abb. 9) (Mansour et al. 2004, Wachowski et al. 2007, Nägerl et al. 2009, Wachowski et al. 2009a, Wachowski et al. 2009b, Wachowski et al. 2010a, Wachowski et al. 2010b, Wachowski et al. 2013) (Abb. 9). Takigawa et al. verwendeten hingegen zwei Behälter, die an nur einem Lastarm befestigt waren (Takigawa et al. 2010). 


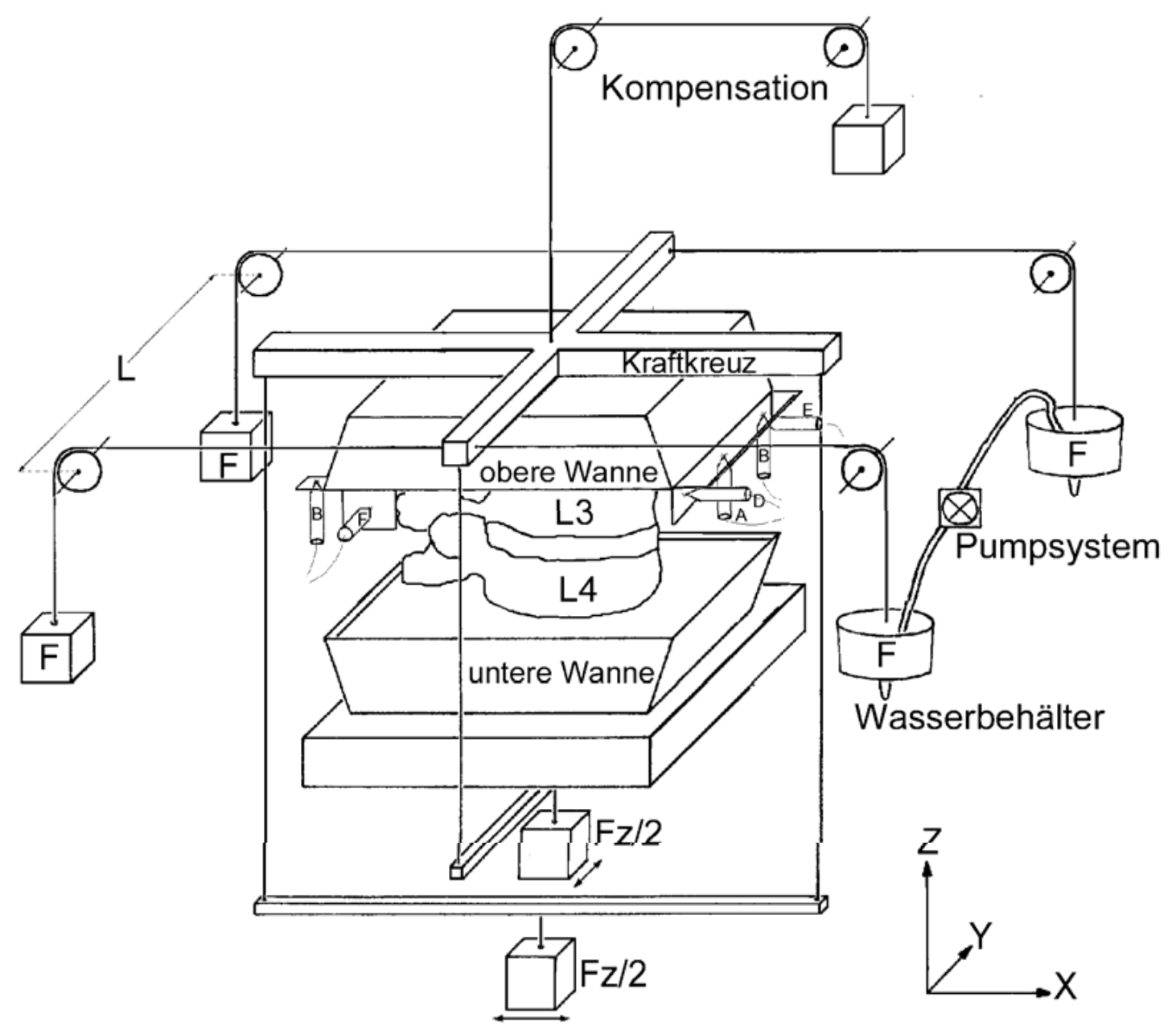

Abb. 9: Beispiel einer Versuchsapparatur (Mansour 2001, S. 73)

In einem Versuch von Dugailly et al. generierten die Autoren die Bewegung durch eine horizontale und eine vertikale Stange. Durch manuelle Verstellung der Stange wurde das Drehmoment appliziert. Entsprechend machten die Autoren keine Angaben zur Größe des Drehmomentes (Dugailly et al. 2010, Dugailly et al. 2011, Dugailly et al. 2013).

Schulze et al. stellten 2012 eine von den bisher genannten Apparaturen stark abweichendes Testgerät vor (Schulze et al. 2012). Es handelte sich um eine Roboterassistierte Testapparatur, die über einen Arm in der Lage war, auf den obersten Wirbel Translationsbewegungen mit einer Genauigkeit von 0,001 +/- 0,0018 mm und 
Rotationsbewegungen mit einer Genauigkeit von 0,025 +/- 0,023 ${ }^{\circ}$ auszuführen (Schulze et al. 2012).

In einer Studie von Goertzen et al. wurde 2004 eine Apparatur vorgestellt (Goertzen et al. 2004), die später von einigen Autoren verwendet wurde (Niosi et al. 2006, Zhu et al. 2007, Zhu et al. 2012). Ein elektrischer Servomotor generierte dabei über einen Gelenkarm lineare Translationsbewegungen. Die Apparatur erlaubte nach Angaben der Autoren 5 DOF und konnte Drehmomente in allen drei Grundbewegungsrichtungen generieren (Goertzen et al. 2004).

Einige Autoren machten keine genaueren Angaben, wie das Drehmoment appliziert wurde (Haher et al. 1992, Cripton et al. 2001, Molnar et al. 2006), geben aber an, ein kontinuierliches Drehmoment generieren zu können (Oxland et al. 1994, Sengupta et al. 2011, Kettler et al. 2004).

Tabelle 1 gibt einen Überblick über die jeweils maximal angelegten Drehmomentwerte, die Bewegungsrichtung, die dem Testobjekt ermöglichten Freiheitsgrade, der Kontinuität der Drehmomentapplikation und der resultierenden Rotationsgeschwindigkeit des Präparates der untersuchten Studien.

Tab. 1 Übersicht über Drehmomentapplikation und Anzahl der DOF in den jeweiligen Studien

\begin{tabular}{|c|c|c|c|c|c|}
\hline & $\begin{array}{l}\text { Maximales } \\
\text { Drehmoment }\end{array}$ & $\begin{array}{l}\text { Bewegungs- } \\
\text { richtung }\end{array}$ & $\begin{array}{l}\text { ermöglichte } \\
\text { Freiheitsgrade } \\
\text { der Apparatur }\end{array}$ & $\begin{array}{l}\text { Applikations- } \\
\text { ablauf des } \\
\text { Drehmomentes } \\
\text { bis } T_{\max }\end{array}$ & $\begin{array}{l}\text { Rotations- } \\
\text { geschwin- } \\
\text { digkeit der } \\
\text { Präparate }\end{array}$ \\
\hline $\begin{array}{l}\text { Bozkus et al. } 2010 \\
\text { erste Testapparatur }\end{array}$ & $+/-7,5 \mathrm{Nm}$ & AR, FI-Ex, LB & k. A. & $\begin{array}{l}1,5 \mathrm{Nm} \text {-Stufen } \\
(45 \mathrm{~s})\end{array}$ & k. A. \\
\hline $\begin{array}{l}\text { Bozkus et al. } 2010 \\
\text { zweite Testappara- } \\
\text { tur }\end{array}$ & $\begin{array}{l}\text { T bis Rotation } \\
\text { aus } 1 \text { Testapp. } \\
\text { erreicht war }\end{array}$ & FI-Ex & k. A. & k. A. & $1 \% \mathrm{~s}$ \\
\hline $\begin{array}{l}\text { Brasiliense et al. } \\
2011\end{array}$ & $+/-7,5$ & AR, FI-Ex, LB & k. A. & $\begin{array}{l}\text { 1,25 Nm-Stufen } \\
(45 \mathrm{~s})\end{array}$ & k. A. \\
\hline
\end{tabular}




\begin{tabular}{|c|c|c|c|c|c|}
\hline Broc et al. 1997 & $+/-4 \mathrm{Nm}$ & AR, FI-Ex, LB & k. A. & $\begin{array}{l}0,5 \mathrm{Nm} \text {-Stufen } \\
(45 \mathrm{~s})\end{array}$ & k. A. \\
\hline Cagli et al. 2004 & $+/-1,5 \mathrm{Nm}$ & AR, FI-Ex, LB & k. A. & $\begin{array}{l}\text { 1.Versuchsdurch- } \\
\text { lauf: } 0,25 \mathrm{Nm}- \\
\text { Stufen }(45 \mathrm{~s}) \\
\text { 2.Versuchsdurchla } \\
\text { uf: kontinuierliche } \\
\text { Bewegung bis } \\
\text { max.T }\end{array}$ & $1-3 \% s$ \\
\hline Chen et al. 2001 & $+/-1.5 \mathrm{Nm}$ & AR, FI-Ex, LB & k. A. & $\begin{array}{l}\text { kontinuierliche } \\
\text { Bewegung bis } \\
\text { max. T }\end{array}$ & k. A. \\
\hline Crawford et al. 2002 & $+/-1,5 \mathrm{Nm}$ & AR, FI-Ex, LB & k. A. & $\begin{array}{l}\text { kontinuierliche } \\
\text { Bewegung bis } \\
\text { max.T }\end{array}$ & k. A. \\
\hline Cripton et al. 2001 & $+2 \mathrm{Nm}$ & $\begin{array}{l}\text { AR nach } \\
\text { rechts }\end{array}$ & k. A. & $\begin{array}{l}\text { 0,25 Nm-Stufen } \\
(30 \mathrm{~s})\end{array}$ & k. A. \\
\hline Cusick et al. 1988 & k. A & $\mathrm{FI}$ & k. A. & k. A. & $1,7 \mathrm{~mm} / \mathrm{s}$ \\
\hline Detwiler et al. 2003 & $+/-5 \mathrm{Nm}$ & AR, FI-Ex, LB & k. A. & $\begin{array}{l}1 \mathrm{Nm}-\text { Stufen } \\
(45 \mathrm{~s})\end{array}$ & k. A. \\
\hline Dickman et al. 1996 & $+/-1,5 \mathrm{Nm}^{*}$ & AR, FI-Ex, LB & k. A. & $\begin{array}{l}0,5 \mathrm{Nm} \text {-Stufen } \\
(60 \mathrm{~s})\end{array}$ & k. A. \\
\hline Dugailly et al. 2010 & k. A. & AR, FI-Ex & k. A. & k. A. & k. A. \\
\hline $\begin{array}{l}\text { Dugailly et al. } 2011 \\
\text { Dugailly et al. } 2013\end{array}$ & & & & & \\
\hline $\begin{array}{l}\text { Grassmann et al. } \\
1998 \\
\text { constrained App. }\end{array}$ & $+/-10 \mathrm{Nm}$ & AR & 1 & $\begin{array}{l}2,5 \mathrm{Nm} \text {-Stufen } \\
(20 \mathrm{~s})\end{array}$ & k. A. \\
\hline $\begin{array}{l}\text { Grassmann et al. } \\
1998 \\
\text { unconstrained App. }\end{array}$ & $+/-10 \mathrm{Nm}$ & $\mathrm{AR}$ & 6 & $\begin{array}{l}2,5 \mathrm{Nm} \text {-Stufen } \\
(20 \mathrm{~s})\end{array}$ & k. A. \\
\hline Haberl et al. 2004 & $\begin{array}{l}+/-12,5 \mathrm{Nm} \\
+/-6 \mathrm{Nm}\end{array}$ & $\begin{array}{l}\text { AR } \\
\text { FI-Ex }\end{array}$ & k. A. & $\begin{array}{l}2,5 \mathrm{Nm} \text {-Stufen } \\
(30 \mathrm{~s}) \\
3 / 6 \mathrm{Nm}\end{array}$ & k. A. \\
\hline Haher et al. 1991 & k. A. & Fl-Ex & k. A. & k. A. & k. A. \\
\hline Haher et al. 1992 & $\begin{array}{l}\text { Rotation erfolg- } \\
\text { te bis } 5^{\circ} \text { und } \\
10^{\circ} \text { gemessen } \\
\text { am oberen } \\
\text { Ende der Appa- } \\
\text { ratur }\end{array}$ & AR & k. A. & k. A. & k. A. \\
\hline
\end{tabular}




\begin{tabular}{|c|c|c|c|c|c|}
\hline Kettler et al. 2004 & $+/-7,5 \mathrm{Nm}$ & AR, FI-Ex, LB & k. A. & $\begin{array}{l}\text { Kontinuierliche } \\
\text { Bewegung bis } \\
\text { max. T }\end{array}$ & $1 \% \mathrm{~s}$ \\
\hline $\begin{array}{l}\text { Lazaro et al. }{ }^{\star \star} 2010 \\
\text { erste Testapparatur }\end{array}$ & $+/-7,5 \mathrm{Nm}$ & AR, FI-Ex, LB & k. A. & $\begin{array}{l}\text { 1,5 Nm- Stufen } \\
(45 \mathrm{~s})\end{array}$ & k. A. \\
\hline $\begin{array}{l}\text { Lazaro et al. } 2010 \\
\text { zweite Testappara- } \\
\text { tur }\end{array}$ & $\begin{array}{l}\text { T bis Rotation } \\
\text { aus } 1 \text { Testapp. } \\
\text { erreicht war }\end{array}$ & FI-Ex & k. A. & k. A. & k. A. \\
\hline Mansour et al. 2004 & $+/-30 \mathrm{Nm}$ & AR & 6 & $\begin{array}{l}\text { kontinuierliche } \\
\text { Bewegung bis } \\
\text { max. T }\end{array}$ & k. A. \\
\hline McNally et al. 2012 & $40 \mathrm{Nm}$ & $\mathrm{FI}$ & k. A. & $\begin{array}{l}10 \mathrm{Nm}, 20 \mathrm{Nm}, \\
30 \mathrm{Nm}, 40 \mathrm{Nm}\end{array}$ & k. A. \\
\hline Molnar et al. 2006 & k. A. & AR & k. A. & k. A. & k. A. \\
\hline Naderi et al. 1998 & $+/-1,5 \mathrm{Nm}$ & AR, FI-Ex, LB & k. A. & $\begin{array}{l}0,25 \mathrm{Nm} \text {-Stufen } \\
(45 \mathrm{~s})\end{array}$ & $10 \% \mathrm{~s}$ \\
\hline Nägerl et al. 2009 & $+/-10 \mathrm{Nm}$ & FI-Ex & 6 & $\begin{array}{l}\text { kontinuierliche } \\
\text { Bewegung bis } \\
\max T \text {. }\end{array}$ & k. A. \\
\hline Niosi et al. 2006 & $+/-7,5 \mathrm{Nm}$ & AR, FI-Ex, LB & 5 & $\begin{array}{l}\text { kontinuierliche } \\
\text { Bewegung bis } \\
\text { max. T. }\end{array}$ & $1,3 \% \mathrm{~s}$ \\
\hline Oxland et al. 1994 & $+/-7,5 \mathrm{Nm}$ & AR, FI-Ex, LB & k. A. & $\begin{array}{l}\text { 4-Stufen } \\
(30 \mathrm{~s})\end{array}$ & k. A. \\
\hline $\begin{array}{l}\text { Rousseau et al. } \\
2006 a\end{array}$ & $\begin{array}{l}\text { k. A. Rotation } \\
\text { erfolgte bis } \\
\text { jeweils } 6^{\circ}\end{array}$ & FI-Ex, LB & 4 & k. A. & k. A. \\
\hline $\begin{array}{l}\text { Rousseau et al. } \\
2006 b\end{array}$ & k. A. & Fl-Ex, LB & 4 & k. A. & k. A. \\
\hline Schulze et al. 2012 & $+/-7,5 \mathrm{Nm}$ & Fl-Ex & 6 & k. A. & $0,25-0,99^{\circ} / \mathrm{s}$ \\
\hline Sengupta et al. 2011 & $+/-10 \mathrm{Nm}$ & AR, FI-Ex, LB & 6 & n. v. & $2,1 \% \mathrm{~s}$ \\
\hline Takigawa et al. 2010 & $+/-7,5 \mathrm{Nm}$ & AR, FI-Ex, LB & k. A. & $\begin{array}{l}0,15 \mathrm{Nm} / \mathrm{s} \\
\text { kontinuierliche } \\
\text { Bewegung bis } \\
\text { max. T }\end{array}$ & k. A. \\
\hline $\begin{array}{l}\text { Wachowski et al. } \\
2007\end{array}$ & ca.+/-1 Nm & AR, FI-Ex, LB & 6 & $\begin{array}{l}\text { kontinuierliche } \\
\text { Bewegung bis } \\
\text { max. T. }\end{array}$ & k. A. \\
\hline $\begin{array}{l}\text { Wachowski et al. } \\
\text { 2009a }\end{array}$ & $\begin{array}{l}+/-32,4 \mathrm{Nm} \\
\text { lumbal } \\
+/-7 \mathrm{Nm} \text { tho- } \\
\text { rakal } \\
+/-1,5 \mathrm{Nm} \\
\text { cervical }\end{array}$ & AR, FI-Ex, LB & 6 & $\begin{array}{l}\text { kontinuierliche } \\
\text { Bewegung bis } \\
\text { max. T }\end{array}$ & k. A. \\
\hline
\end{tabular}




\begin{tabular}{|c|c|c|c|c|c|}
\hline $\begin{array}{l}\text { Wachowski et al. } \\
2009 \text { b }\end{array}$ & $\begin{array}{l}+/-32,50 \mathrm{Nm} \\
+/-25 \mathrm{Nm} \\
+/-13,5 \mathrm{Nm}\end{array}$ & AR & 6 & $\begin{array}{l}\text { kontinuierliche } \\
\text { Bewegung bis } \\
\text { max. T. }\end{array}$ & k. A. \\
\hline $\begin{array}{l}\text { Wachowski et al. } \\
2010 a\end{array}$ & $\begin{array}{l}+/-32,4 \mathrm{Nm} \\
\text { lumbal } \\
+/-7 \mathrm{Nm} \text { tho- } \\
\text { rakal }\end{array}$ & AR, FI-Ex & 6 & $\begin{array}{l}\text { kontinuierliche } \\
\text { Bewegung bis } \\
\text { max. T. }\end{array}$ & k. A. \\
\hline $\begin{array}{l}\text { Wachowski et al. } \\
\text { 2010b }\end{array}$ & $\begin{array}{l}+/-32,40 \mathrm{Nm} \\
+/-25 \mathrm{Nm}\end{array}$ & AR & 6 & $\begin{array}{l}\text { kontinuierliche } \\
\text { Bewegung bis } \\
\text { max. T }\end{array}$ & k. A. \\
\hline $\begin{array}{l}\text { Wachowski et al. } \\
2013\end{array}$ & $+/-2,24 \mathrm{Nm}$ & AR & 6 & $\begin{array}{l}\text { kontinuierliche } \\
\text { Bewegung bis } \\
\text { max. T }\end{array}$ & k. A. \\
\hline $\begin{array}{l}\text { Zhu et al. } \\
2007\end{array}$ & $+/-10 \mathrm{Nm}$ & AR, FI-Ex, LB & 5 & $\begin{array}{l}\text { kontinuierliche } \\
\text { Bewegung bis } \\
\text { max. T }\end{array}$ & $2 \% \mathrm{~s}$ \\
\hline $\begin{array}{l}\text { Zhu et al. } \\
2012\end{array}$ & $+/-5 \mathrm{Nm}$ & AR, FI-Ex, LB & 5 & $\begin{array}{l}\text { kontinuierliche } \\
\text { Bewegung bis } \\
\text { max. T }\end{array}$ & $0,5^{\circ} / \mathrm{s}$ \\
\hline
\end{tabular}

* Bei verschiedenen Drehmomentmaximalwerten für verschiedene Zustände des Präparates wird jeweils der maximale Wert für die intakten Präparate angegeben.

** Es erfolgte keine Ermittlung kinematischer Daten. Der Versuch ist aber Grundlage für weiteren Testdurchläufe in denen kinematische Daten erhoben werden.

AR axiale Rotation, Fl-Ex Flexion-Extension, LB laterale Beugung, k. A. keine Angabe, n. v. nicht verwendet 


\subsection{Vorlast}

Die an die Segmente ansetzenden Muskelkräfte und das Körpergewicht wurden in einem Teil der untersuchten Studien durch eine Vorlast (preload) simuliert. Die Autoren, die eine Vorlast generierten, verwendeten dazu verschiedene Verfahren. Allen Verfahren ist gemein, dass das Segment dabei komprimiert bzw. gestaucht wird. Unterschiede bestehen jedoch im Ansatzpunkt und Verlauf des Kraftvektors.

Häufig findet sich in der Literatur der Oberbegriff compressive preload. Die resultierende Kraft konnte dabei auf verschiedene Weise erzeugt werden:

So applizierten Naderi et al. durch das Eigengewicht des oberen Befestigungsbehälters eine kompressive Vorlast von 5,2 N (Naderi et al. 1998). Grassmann et al. erzeugten in einer der verwendeten Testapparaturen kompressive Kräfte von etwa $25 \mathrm{~N}$ mittels eines vertikalen Kolbens, der auf den kranialen Wirbel einwirkte (s. Kap. 3.2.2). Zusätzlich entstanden nach Angaben der Autoren Kompressionskräfte während eine axiale Rotation erfolgte, da vertikale Bewegungen des Segmentes durch die starre Anordnung der Testapparatur unterbunden wurden. Es wirkten somit kompressive Kräfte von $100 \mathrm{~N}$ bis $150 \mathrm{~N}$ (Grassmann et al. 1998). Cusick et al. verwendeten ebenfalls einen vertikal ausgerichteten Kolben, der auf den kranialen Wirbel einwirkte und nach Verschiebung nach anterior in der Sagittalebene neben dem Flexionsmoment auch eine Vorlast applizierte. Zur Höhe der Vorlast machten die Autoren keine Angaben (Cusick et al. 1988).

Takigawa et al. führten die Versuche mit und ohne Vorlast durch. Für den Testdurchlauf mit Vorlast wurden vier an einer Platte über dem obersten Segment angebrachte Gewichte verwendet, um die kompressive Kraft auf das Präparat zu generieren. Die Kabel waren an jeder Ecke der Platte angebracht und sollten nach Angaben der Autoren das Segment gleichmäßig mit einer Vorlast von $400 \mathrm{~N}$ belasten ohne Scherkräfte oder Drehmomente zu generieren (Takigawa et al. 2010).

Cripton et al. untersuchten in einer Studie verschiedene Formen der Vorlastapplikation, indem sie den Verlauf des Kraftvektors der Vorlast entlang des Präparates veränderten (Cripton et al. 2000) (s. Kap. 4.3). An Kabeln befestigte seitlich des Präparates zunächst frei herabhängende Gewichte generierten dabei die zur Vorlastapplikation nötige Kraft. Dabei sei der Verlauf des Kraftvektors abhängig vom Verlauf der Kabel und somit der Stellung des Präparates. Bei Bewegung des Präparates können so durch Veränderung des Verlaufs des Kraftvektors zusätzliche Drehmomente ge- 
neriert werden, die von den Autoren als Artefaktmomente bezeichnet wurden. Der Verlauf der Kabel und somit des Kraftvektors wurde in den Versuchsdurchläufen durch Führungen, entlang des Präparates beeinflusst (Cripton et al. 2000). Haberl et al. (2004) folgten einer der möglichen Versuchsanordnungen von Cripton et al. (2000) und applizierten die Vorlast durch über dem obersten Wirbel befestigte, seitlich der Segmente herabhängende Gewichte (s. Abb. 12). Der Befestigungspunkt der Gewichte war dabei nach anterior und posterior verschiebbar. Wurde der Applikationspunkt nach anterior bzw. posterior verschoben, resultierten zu gleich Flexionsund Extensionsdrehmomente, die durch eine load cell gemessen und auf 3 bzw. 6 $\mathrm{Nm}$ begrenzt wurden (s.a. 3.2 Drehmomentapplikation) (Haberl et al. 2004).

Wilder et al. führten an, dass der Kraftvektor stets durch das Zentrum der Wirbel, dem sogenannten balance point verlaufen müsse, um durch den Kraftvektor der Vorlast kein Artefaktmoment zu erzeugen (Wilder et al. 1989). Dieser Bereich wird auch als Widerstandszentrum bezeichnet (Nägerl et al. 2009). Einige Autoren nutzten die Verschiebung des Kraftvektors jedoch gezielt, um Flexions- oder Extensionsmomente zu erzeugen (McNally et al. 2012). Dies geschah über eine axiale Belastung der oberen Befestigungsplatte, über der eine entlang der Mittsagittalen verschiebbare Rolle angebracht war (s. Abb. 10). Somit konnte der Applikationspunkt der Vorlast verändert und zugleich ein Flexionsmoment generiert werden.

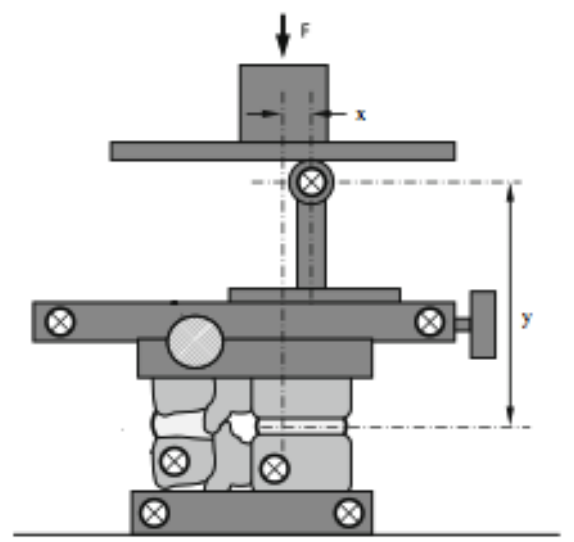

Abb. 10 Beispiel einer Versuchsapparatur (McNally et al. 2012, S. 613; mit freundlicher Erlaubnis von Springer Science and Business Media)

Auch Lazaro et al. (2010) und Bozkus et al. (2010) applizierten Drehmoment und Vorlast über gleiche Apparaturbestandteile in einer der jeweils verwendeten Testapparaturen. Ein Riemen, der anterior bzw. posterior am oberen Ende des Präparats befestigt war und von einem Motor gespannt bzw. entspannt werden konnte, 
applizierte Flexions- und Extensionsmomente (s. Kap. 3.2). Zugleich wirkte durch Spannung der Riemen eine kompressive Vorlast von 400 N. Lazaro et al. nannten die angewandte Vorlast compressive follower load, Bozkus et al. führten für die gleiche Form der Vorlastapplikation den Begriff compressive load an. In der ersten verwendeten Testapparatur hatten die Autoren zunächst keine Vorlast appliziert (Bozkus et al. 2010, (Lazaro et al. 2010).

Rousseau et al. applizierten eine kompressive Vorlast von $850 \mathrm{~N}$. Da Vorlastvektor und Wirbel in einem $40^{\circ}$ Winkel zueinander standen, resultierten nach Angaben der Autoren Kompressionskräfte in Höhe von 650 N sowie horizontale Scherkräfte in Höhe von $550 \mathrm{~N}$ (Rousseau et al. 2006a, Rousseau et al. 2006b). Mit dem $40^{\circ}$ Winkel sollte nach Angaben der Autoren die „Sakralneigung“ der Segmente physiologisch dargestellt werden.

Haher et al. generierten die Vorlast (max.: $1250 \mathrm{~N}$ ), in dem sie den vertikalen Abstand zwischen den Befestigungsplatten verringerten und somit das Segment stauchten. Durch Keile, die am oberen Wirbel angebracht waren, konnten die Kompressionskräfte zu gleich zur Applikation von Flexions- und Extensionsmomenten genutzt werden (Haher et al. 1991).

Seligman et al. führten Ihre Versuche sowohl mit als auch ohne Vorlast durch. Für den Testdurchlauf mit applizerter Vorlast erfolgten aber nur Angaben zur Höhe der kompressiven Vorlast (ca.300 N). Wie diese appliziert wurde, wurde nicht angegeben (Seligman et al. 1984).

Einige Autoren applizierten die Vorlast über ein am oberen Befestigungsbehälter angebrachtes Stahlkreuz, das auch zur Drehmomentapplikation genutzt wurde (s. Kap. 3.2). Über Kabel, die an den vier Enden des Stahlkreuzes angebracht waren, wurden zwei Schienen gehalten. An diesen Schienen war jeweils ein entlang der Achse verschiebbares Gewicht angebracht. Die Gewichte konnten mit einer Genauigkeit von +/- 0,5 mm verschoben werden. Variationen der Vorlastposition und Vorlastgrößen wurden nicht untersucht (Mansour et al. 2004). Einige Autoren verwendeten die gleiche Apparatur und verschoben die Gewichte innerhalb der Sagittalebene (Wachowski et al. 2007 , Nägerl et al. 2009, Wachowski et al. 2009a, Wachowski et al. 2009b, Wachowski et al. 2010, Wachowski et al. 2010, Wachowski et al. 2013) und induzierten damit nach eigenen Angaben ein zusätzliches Flexions- und Exten- 
sionsmoment. So gaben Nägerl et al. (2009) an, dass bei dorsal Verlagerung der Vorlast (200 N) ein zusätzliches Extensionsmoment von 8 Nm entstand. Weitere Angaben zu den jeweiligen Vorlastgrößen finden sich in Tab. 2.

Eine terminologische Abgrenzung zur compressive load stellt die follower preload dar. Auch bei dieser Form der Vorlastapplikation handelt es sich um eine kompressive Belastung der Segmente. Dabei sollen Artefaktmomente, wie von Cripton et al. (2000) (s.o.) angeführt, verhindert werden (Zhu et al. 2007). Dabei entspricht die Richtung des Kraftvektors nicht mehr der Richtung des Gravitationsvektors, sondern verläuft tangential entlang der zuvor ermittelten center of rotations (COR) der Segmente (Patwardhan et al. 1999) (s. Abb. 11).

Diesem Prinzip folgten einige Autoren und applizierten eine Vorlast in Höhe $600 \mathrm{~N}$ (Zhu et al. 2007), $400 \mathrm{~N}$ (Zhu et al. 2012), bzw. $200 \mathrm{~N}$ (Sengupta et al. 2011). Niosi et al. (2006) führten die Versuche mit $600 \mathrm{~N}$ follower preload und ohne Vorlast durch.

Nicht in allen Studien wurde eine Vorlast appliziert. So verzichteten einige Autoren bewusst auf die Applikation einer Vorlast, um die Reproduzierbarkeit der sonstigen Messungen zu erhöhen (Cripton et al. 2001, Brasiliense et al. 2011, Schulze et al. 2012).

Dugailly et al. versuchten hingegen gezielt Kompressionskräfte zu verringern, indem sie das Präparat mit dem kranialen Ende auf den Boden der Apparatur drehten und die Bewegung am nun oben gelegenen, kaudalen Wirbel erfolgen ließen (Dugailly et al. 2010, Dugailly et al. 2011, Dugailly et al. 2013). 


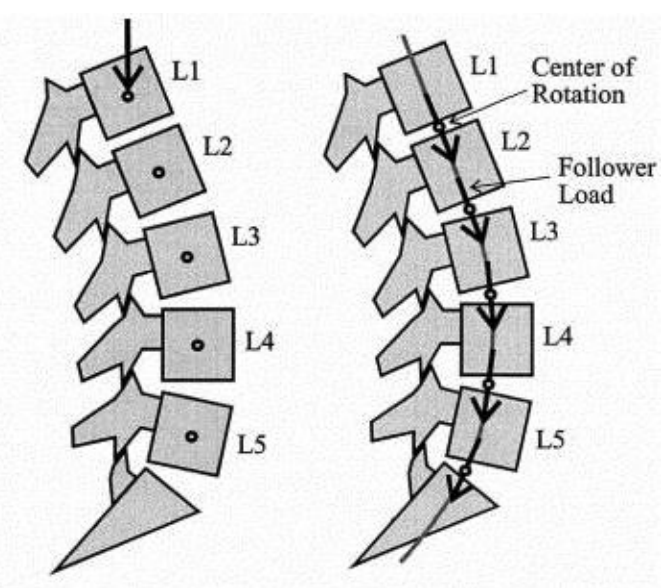

Abb. 11 zwei Formen der Vorlastapplikation, links vertikale Belastung, rechts follower preload (Patwardhan et al. 1999, S. 1004, mit Erlaubnis von Lipincott Williams and Wilkins/ Wolters Kluwer Health)

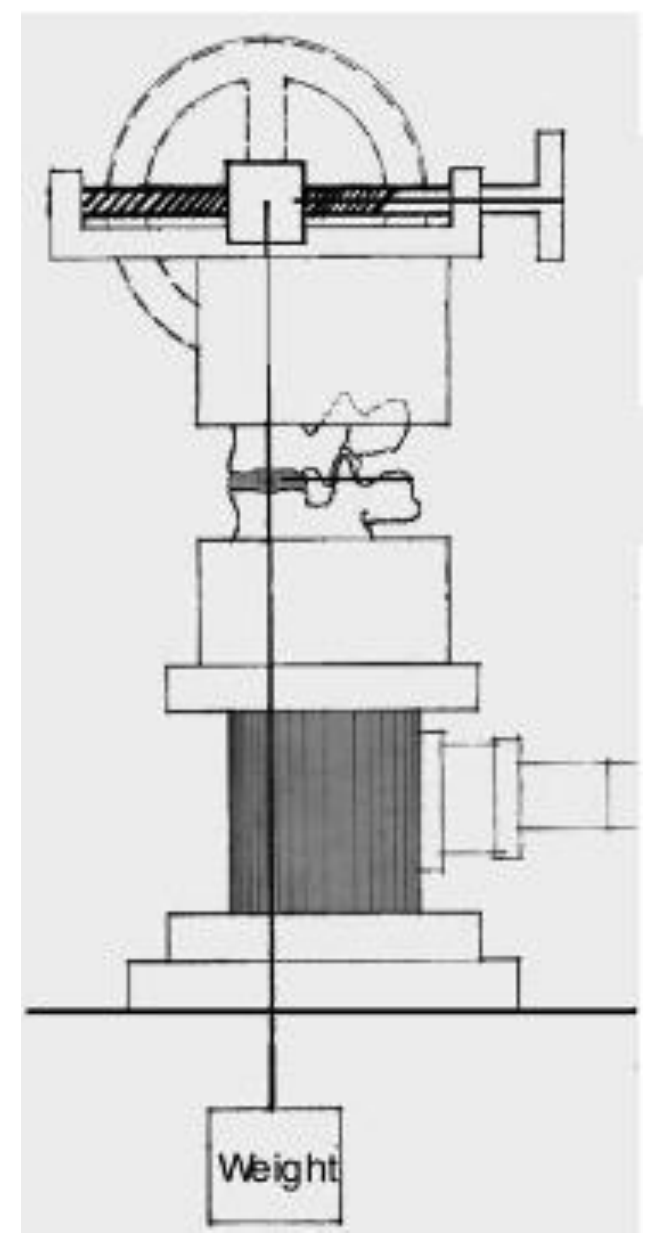

Abb. 12 Beispiel einer Versuchsapparatur (Haberl et al. 2004, S. 562, mit freundlicher Erlaubnis von Springer Science and Business Media) 
Tabelle 2 fasst die verschiedenen Arten der Vorlastapplikation sowie die verwendeten Vorlastgrößen zusammen.

Tab. 2 Art der Vorlastapplikation und Vorlastgröße in den untersuchten Studien

\begin{tabular}{|c|c|c|}
\hline & $\begin{array}{l}\text { Vorlastart nach Angaben } \\
\text { der Autoren }\end{array}$ & Vorlastgrößen \\
\hline $\begin{array}{l}\text { Bozkus et al. } 2010 \\
\text { erste Testapparatur }\end{array}$ & n. v. & n. v. \\
\hline $\begin{array}{l}\text { Bozkus et al. } 2010 \\
\text { zweite Testapparatur }\end{array}$ & $\begin{array}{l}\text { compressive load über } \\
\text { Stauchung des Präparates } \\
\text { durch Riemen (s. Text) }\end{array}$ & $400 \mathrm{~N}$ \\
\hline $\begin{array}{l}\text { Brasiliense et al. } \\
2010\end{array}$ & n. v. & n. v. \\
\hline Broc et al. 1997 & k. A. & k. A. \\
\hline Cagli et al. 2004 & k. A. & k. A. \\
\hline Chen et al. 2001 & k. A. & k. A. \\
\hline Crawford et al. 2002 & k. A. & k. A. \\
\hline Cripton et al. 2001 & k. A. & k. A. \\
\hline Cusick et al. 1988 & $\begin{array}{l}\text { compressive preload über } \\
\text { vertikalen Kolben (s. Text) }\end{array}$ & K.A. \\
\hline Detwiler et al. 2003 & k. A. & k. A. \\
\hline Dickman et al. 1996 & k. A. & k. A. \\
\hline Dugailly et al. 2010 & n. v. & n. v. \\
\hline \begin{tabular}{|l|} 
Dugailly et al. 2011 \\
Dugailly et al. 2013
\end{tabular} & & \\
\hline $\begin{array}{l}\text { Grassmann et al. } \\
1998 \\
\text { constrained app. }\end{array}$ & $\begin{array}{l}\text { compressive load über } \\
\text { Stauchung des Präparates } \\
\text { durch starre Befestigungs- } \\
\text { platten (s. Text) }\end{array}$ & $\begin{array}{l}\text { initial <25 N, Anstieg bei } \\
\text { vertikaler Bewegung des } \\
\text { Segmentes aufgrund star- } \\
\text { rer Anordnung der Test- } \\
\text { apparatur auf bis zu } 150 \mathrm{~N}\end{array}$ \\
\hline $\begin{array}{l}\text { Grassmann et al.1998 } \\
\text { unconstrained app. }\end{array}$ & k. A. & k. A. \\
\hline Haberl et al. 2004 & $\begin{array}{l}\text { compressive preload durch } \\
\text { herabhängende Gewichte } \\
\text { (s. Text) }\end{array}$ & 0 und $200 \mathrm{~N}$ \\
\hline Haher et al. 1991 & $\begin{array}{l}\text { compressive load über } \\
\text { vertikale Höhenverände- } \\
\text { rung der Befestigungsplat- } \\
\text { ten (s. Text) }\end{array}$ & $\begin{array}{l}100 \mathrm{~N}-\text { Stufen bis max. } \\
1250 \mathrm{~N}\end{array}$ \\
\hline Haher et al. 1992 & n. v. & n. v. \\
\hline Kettler et al. 2004 & n. v. & n. v. \\
\hline $\begin{array}{l}\text { Lazaro et al. }{ }^{* \star} 2010 \\
\text { erste Testapparatur }\end{array}$ & n. v. & n. v. \\
\hline $\begin{array}{l}\text { Lazaro et al. } 2010 \\
\text { zweite Testapparatur }\end{array}$ & $\begin{array}{l}\text { compressive follower load } \\
\text { über Stauchung des Prä- } \\
\text { parates durch Riemen } \\
\text { (s.Text) }\end{array}$ & $400 \mathrm{~N}$ \\
\hline Mansour et al. 2004 & $\begin{array}{l}\text { VL- Applikation über frei } \\
\text { hängende Gewichte unter } \\
\text { dem Segment (s.Text) }\end{array}$ & $200 \mathrm{~N}$ \\
\hline
\end{tabular}




\begin{tabular}{|c|c|c|}
\hline McNally et al. 2012 & $\begin{array}{l}\text { compressive load über } \\
\text { vertikale Krafteinwirkung } \\
\text { auf obere Befestigungs- } \\
\text { platte (s. Text) }\end{array}$ & $250 \mathrm{~N} / \mathrm{s}$ bis max. $1000 \mathrm{~N}$ \\
\hline Molnar et al. 2006 & k. A. & k. A. \\
\hline Naderi et al. 1998 & $\begin{array}{l}\text { compressive preload durch } \\
\text { Eigengewicht des oberen } \\
\text { Befestigungsbehälters (s. } \\
\text { Text) }\end{array}$ & $5,2 \mathrm{~N}$ \\
\hline Nägerl et al. 2009 & $\begin{array}{l}\text { VL- Applikation über frei } \\
\text { hängende Gewichte unter } \\
\text { dem Segment (s.Text) }\end{array}$ & $200 \mathrm{~N}$ \\
\hline Niosi et al. 2006 & follower preload (s. Text) & $0 \mathrm{~N}$ und $600 \mathrm{~N}$ \\
\hline Oxland et al. 1994 & k. A. & k. A. \\
\hline Rousseau et al. 2006 & $\begin{array}{l}\text { compressive preload } \\
\text { (s.Text) über vertikal ein- } \\
\text { wirkende Kraft auf obere } \\
\text { Befestigungsplatte (s.Text) }\end{array}$ & $\begin{array}{l}850 \mathrm{~N} \text { resultieren in } 650 \mathrm{~N} \\
\text { Kompression und } 550 \mathrm{~N} \\
\text { Scherkräfte }\end{array}$ \\
\hline Rousseau et al. 2006 & $\begin{array}{l}\text { compressive preload über } \\
\text { vertikal einwirkende Kraft } \\
\text { auf obere Befestigungs- } \\
\text { platte } \\
\text { (s. Text) }\end{array}$ & $\begin{array}{l}850 \mathrm{~N} \text { resultieren in } 650 \mathrm{~N} \\
\text { Kompression und } 550 \mathrm{~N} \\
\text { Scherkräfte }\end{array}$ \\
\hline Schulze et al. 2012 & n. v. & n. v. \\
\hline Seligman et al. 1984 & k. A. & 0 und ca. $300 \mathrm{~N}$ \\
\hline Sengupta et al. 2011 & follower preload (s. Text) & $200 \mathrm{~N}$ \\
\hline Takigawa et al. 2010 & $\begin{array}{l}\text { compressive preload } \\
\text { über von oberer Befesti- } \\
\text { gungsplatte herabhängen- } \\
\text { de Gewichte (s. Text) }\end{array}$ & $0 \mathrm{~N}$ und $400 \mathrm{~N}$ \\
\hline $\begin{array}{l}\text { Wachowski et al. } \\
2007\end{array}$ & $\begin{array}{l}\text { VL- Applikation über frei } \\
\text { hängende Gewichte unter } \\
\text { dem Segment (s. Text) }\end{array}$ & $20 \mathrm{~N}$ \\
\hline $\begin{array}{l}\text { Wachowski et al. } \\
2009 a\end{array}$ & $\begin{array}{l}\text { VL- Applikation über frei } \\
\text { hängende Gewichte unter } \\
\text { dem Segment (s. Text) }\end{array}$ & $\begin{array}{l}\text { Varaiationen von: } \\
0 \mathrm{~N} \text { bis } 70 \mathrm{~N} \text { zervikal } \\
0 \mathrm{~N} \text { bis } 90 \mathrm{~N} \text { thorakal } \\
0 \mathrm{~N} \text { bis } 300 \mathrm{~N} \text { lumbal }\end{array}$ \\
\hline $\begin{array}{l}\text { Wachowski et al. } \\
2009 b\end{array}$ & $\begin{array}{l}\text { VL- Applikation über frei } \\
\text { hängende Gewichte unter } \\
\text { dem Segment (s. Text) }\end{array}$ & 200 und $400 \mathrm{~N}$ \\
\hline $\begin{array}{l}\text { Wachowski et al. } \\
2010 a\end{array}$ & $\begin{array}{l}\text { VL- Applikation über frei } \\
\text { hängende Gewichte unter } \\
\text { dem Segment (s. Text) }\end{array}$ & $\begin{array}{l}\text { Variationen von: } \\
0 \mathrm{~N} \text { bis } 90 \mathrm{~N} \text { thorakal und } \\
0 \mathrm{~N} \text { bis } 300 \mathrm{~N} \text { lumbal }\end{array}$ \\
\hline $\begin{array}{l}\text { Wachowski et al. } \\
\text { 2010b }\end{array}$ & $\begin{array}{l}\text { VL- Applikation über frei } \\
\text { hängende Gewichte unter } \\
\text { dem Segment (s. Text) }\end{array}$ & $200 \mathrm{~N}, 300 \mathrm{~N}, 400 \mathrm{~N}$ \\
\hline $\begin{array}{l}\text { Wachowski et al. } \\
2013\end{array}$ & $\begin{array}{l}\text { VL- Applikation über frei } \\
\text { hängende Gewichte unter } \\
\text { dem Segment (s. Text) }\end{array}$ & $20 \mathrm{~N}$ \\
\hline Zhu et al. 2007 & follower preload (s. Text) & $600 \mathrm{~N}$ \\
\hline Zhu et al. 2012 & follower preload (s. Text) & $400 \mathrm{~N}$ \\
\hline
\end{tabular}




\subsection{Verwendete Präparate}

In allen untersuchten Studien wurden Präparate von menschlichen Spendern untersucht. Lediglich Schulze et al. griffen auf tierische Präparate zurück (Schulze et al. 2012). Zwischen den untersuchten Präparaten lassen sich zahlreiche Unterscheidungen treffen (Tab. 3). So wurden zervikale, thorakale sowie lumbale Segmente untersucht, wobei lumbale Segmente am häufigsten Gegenstand der Untersuchungen waren (Tab. 3). Das Alter der getesteten menschlichen Segmente variierte dabei von 28 Jahren (Haberl et al. 2004) bis 99 Jahren (Takigawa et al. 2010) und die Anzahl der getesteten Präparate von einem (Cripton et al. 2001) bis 47 (Seligman et al. 1984).

Vor der Verwendung für den Testdurchlauf wurden röntgenologisch pathologische Erscheinungen ausgeschlossen (z. B. Cusick et al. 1988, Oxland et al. 1994, Dickman et al. 1996, Broc et al. 1997, Grassmann et al. 1998, Naderi et al. 1998, Haberl et al. 2004, Wachowski et al. 2007, Lazaro et al. 2010, Takigawa et al. 2010 und andere).

Einige Autoren verwendeten ausschließlich Präparate ohne pathologische Erscheinungen, machten jedoch keine Angaben, wie dies zuvor festgestellt wurde (Haher et al. 1991, Mansour et al. 2004). Seligman et al. untersuchten die Präparate röntgenologisch auf vorliegende pathologische Veränderungen, schlossen diese jedoch nicht vom Versuchsdurchlauf aus, sondern untersuchten das biomechechanische Verhalten der Segmente im Vergleich zu pathologsich unauffälligen Präparaten (Seligman et al. 1984).

Einige Autoren machten keine Angaben, ob pathologische Erscheinungen der Präparate festgestellt wurden und zum Ausschluss vom Testdurchlauf führten (z. B. Cripton Cripton et al. 2001, Kettler et al. 2004, Niosi et al. 2006, Zhu et al. 2007, Dugailly et al. 2011, Sengupta et al. 2011, McNally et al. 2012, Schulze et al. 2012, Zhu et al. 2012 und andere).

Entsprechend den unterschiedlichen Zielstellungen der untersuchten Studien (Kap. 3.1) wurden die Präparate von den Autoren in verschiedenen Zuständen getestet.

Einige Autoren verfolgten das Ziel, in Bezug auf den anatomischen Zustand der Präparate möglichst physiologische Bedingungen zu erhalten (Tab. 3). Die Präparate werden in Tabelle 3 als „physiologisch“ bezeichnet (s. Tab 3). Andere Autoren resezierten bewusst anatomische Strukturen, um gezielt auch patho- 
logische Bedingungen zu testen (Tab. 3). Der Zustand der Präparate wird in Tab. 3 als „pathologisch“ bezeichnet.

Einige Autoren testeten zusätzlich auch Implantatsysteme, Fusion der Bewegungssegmente oder andere Fixierungsmethoden (Tab. 3). Auf die zahlreichen Formen der Implantatsysteme und die verschiedenen Möglichkeiten der Fusion von Bewegungssegmenten soll, entsprechend der Zielstellung der vorliegenden Studie, nicht näher eingegangen werden. In Tabelle 3 ist für die jeweiligen Präparate, mit denen ein Implantat, Fusion oder andere Fixierungsmaßnahmen, der Vermerk "Implantatsystem“, „Fusion“ oder „Fixierung“ zu finden (Tab. 3).

Um in Bezug auf den anatomischen Zustand der Präparate physiologische Bedingungen erhalten zu können wurde von den Autoren das generelle Prinzip verfolgt, das Bewegungssegment (s. Kap. 3.4) intakt zulassen, jedoch das Muskelgewebe zu entfernen (z. B. Dickman et al. 1996, Naderi et al. 1998, Cripton et al. 2001, Haberl et al. 2004, Niosi et al. 2006, Rousseau 2006b, Zhu et al. 2007, Bozkus et al. 2010, Takigawa et al. 2010, McNally et al. 2012 und andere).

Nur in wenigen Fällen wurden einzelne Muskelgruppen (interkostale Muskulatur) (Brasiliense et al. 2011) oder suboccipitale Muskeln belassen (Dugailly et al. 2010, Dugailly et al. 2011, Dugailly et al. 2013).

Einige Autoren machten keine Angaben, wie die Präparate für den Testdurchlauf präpariert wurden (Cusick et al. 1988, Mansour et al. 2004, Wachowski et al. 2007, Wachowski et al. 2009a, Wachowski et al. 2009b, Sengupta et al. 2011, Schulze et al. 2012). Nach Präparation der Testobjekte folgten im weiteren Versuchsdurchlauf in einigen Fällen weitere Veränderungen hinsichtlich des anatomischen Zustands des Präparates. In diesen Fällen gibt Tabelle 3 einen Überblick, in welchem Zustand die Präparate im weiteren Testdurchlauf getestet wurden. 
Tab. 3 Verwendete Präparate in den untersuchten Studien

\begin{tabular}{|c|c|c|c|c|c|}
\hline & $\begin{array}{l}\text { Anzahl der } \\
\text { Präparate }\end{array}$ & $\begin{array}{l}\text { Untersuchte } \\
\text { Level der } \\
\text { Präparate }\end{array}$ & $\begin{array}{l}\text { Anzahl der in } \\
\text { die Testappara- } \\
\text { tur eingespann- } \\
\text { ten Segmente }\end{array}$ & $\begin{array}{l}\text { durchschnitt- } \\
\text { liches Alter } \\
\text { der Präparate } \\
\text { (min. Alter- } \\
\text { max. Alter) }\end{array}$ & $\begin{array}{l}\text { Unterteilung des } \\
\text { Zustands der Präpa- } \\
\text { rate nach Angaben } \\
\text { der Autoren }\end{array}$ \\
\hline Bozkus et al. 2010 & 7 & L3-S1 & trisegmental & $\begin{array}{l}58 \text { Jahre } \\
\text { (32-71 Jahre) }\end{array}$ & $\begin{array}{l}\text { physiologisch, } \\
\text { pathologisch lam- } \\
\text { inektomiech, screw } \\
\text { rod fixation }\end{array}$ \\
\hline Brasiliense et al. 2011 & 8 & $\begin{array}{l}\text { T2-T5 } \\
\text { T3-T6 } \\
\text { T4-T7 }\end{array}$ & trisegmental & $\begin{array}{l}\text { 62 Jahre } \\
\text { (36-92 Jahre) }\end{array}$ & $\begin{array}{l}\text { physiologisch, } \\
\text { pathologisch }\end{array}$ \\
\hline Broc et al. 1997 & 8 & T4-12 & $\begin{array}{l}\text { poly-/ mono- } \\
\text { segmental }\end{array}$ & $\begin{array}{l}\text { 62 Jahre } \\
\text { (40-75 Jahre) }\end{array}$ & $\begin{array}{l}\text { physiologisch, Micro- } \\
\text { discectomy }\end{array}$ \\
\hline Cagli et al. 2004 & 6 & C3-T1 & polysegmental & $\begin{array}{l}\text { 59,5 Jahre } \\
\text { (51-66 Jahre) }\end{array}$ & $\begin{array}{l}\text { physiologisch patho- } \\
\text { logisch Fixierung }\end{array}$ \\
\hline Chen et al. 2001 & 7 & C4-T1 & polysegmental & 53-66 Jahre & $\begin{array}{l}\text { physiologisch } \\
\text { pathologisch }\end{array}$ \\
\hline Crawford et al. 2002 & 9 & C4-C7 & polysegmental & 52,6 Jahre & $\begin{array}{l}\text { physiologisch } \\
\text { pathologisch }\end{array}$ \\
\hline Cripton et al. 2001 & 1 & C2-C3 & monosegmental & k. A. & physiologisch \\
\hline Cusick et al. 1988 & 12 & $\begin{array}{l}\mathrm{C} 3-\mathrm{C} 4 \\
\mathrm{C} 6-\mathrm{C} 7\end{array}$ & monosegmental & $\begin{array}{l}\text { 71,7 Jahre } \\
\text { (65-84 Jahre) }\end{array}$ & $\begin{array}{l}\text { physiologisch patho- } \\
\text { logisch Fixierung }\end{array}$ \\
\hline Detwiler et al. 2003 & 5 & $\begin{array}{l}\text { L1-L5 } \\
\text { L1-L4 } \\
\text { L2-L5 }\end{array}$ & polysegmental & $\begin{array}{l}\text { 54,2 Jahre } \\
\text { (26-64 Jahre) }\end{array}$ & $\begin{array}{l}\text { physiologisch, } \\
\text { Christmas-tree lami- } \\
\text { nectomy, facet- } \\
\text { sparing laminectomy }\end{array}$ \\
\hline Dickman et al. 1996 & $4 \mathrm{FSU}$ & $\mathrm{C} 1-\mathrm{C} 2$ & monosegmental & $\begin{array}{l}\text { 62 Jahre } \\
\text { (54-72Jahre) }\end{array}$ & $\begin{array}{l}\text { physiologisch } \\
\text { pathologisch } \\
\text { cable fixation }\end{array}$ \\
\hline Dugailly et al. 2010 & 10 & $\mathrm{C} 0-\mathrm{C} 3$ & polysegmental & k. A. & physiologisch \\
\hline Dugailly et al. 2011 & 10 & C0-C3 & polysegmental & k. A & pyhysiologisch \\
\hline Dugailly et al. 2013 & 1 & C0-C3 & polysegmental & k. A. & physiologisch \\
\hline Grassmann et al. 1998 & $10 \mathrm{FSU}$ & $\begin{array}{l}\text { L3-L4 } \\
\text { L5-S1 }\end{array}$ & monosegmental & (31-55 Jahre) & physiologisch \\
\hline Haberl et al. 2004 & 10 & L3-L5 & bisegmental & $\begin{array}{l}\text { 55 Jahre } \\
\text { (28-69Jahre) }\end{array}$ & physiologisch \\
\hline
\end{tabular}




\begin{tabular}{|c|c|c|c|c|c|}
\hline Haher et al. 1991 & 10 & T1-S3 & polysegmental & k. A. & $\begin{array}{l}\text { physiologisch, patho- } \\
\text { logisch }\end{array}$ \\
\hline Haher et al. 1992 & 10 & T1-S1 & polysegmental & k. A. & $\begin{array}{l}\text { physiologisch, patho- } \\
\text { logisch }\end{array}$ \\
\hline Kettler et al. 2004 & 11 & L4-L5 & monosegmental & 52 Jahre & $\begin{array}{l}\text { physiologisch } \\
\text { pathologisch } \\
\text { Implantatsystem }\end{array}$ \\
\hline Lazaro et al. ${ }^{* \star} 2010$ & 7 & T12-L2 & bisegmental & $\begin{array}{l}57 \text { Jahre } \\
\text { 36-82 Jahre }\end{array}$ & $\begin{array}{l}\text { physiologisch } \\
\text { Implantatsystem }\end{array}$ \\
\hline Mansour et al. 2004 & 2 & L3-L4 & monosegmental & k. A. & physiologisch \\
\hline McNally et al. 2012 & 8 & $\begin{array}{l}\text { L3-L4 } \\
\text { L4-L5 }\end{array}$ & monosegmental & $\begin{array}{l}\text { 76,7 Jahre } \\
\text { (Standardab- } \\
\text { weicung: } \\
\text { +/-13 Jahre) }\end{array}$ & $\begin{array}{l}\text { physiologisch, Im- } \\
\text { plantatsystem }\end{array}$ \\
\hline Molnar et al. 2006 & 24 & $\begin{array}{l}\text { thorakale } \\
\text { Segmente k. } \\
\text { weiteren An- } \\
\text { gaben }\end{array}$ & k. A. & 76 Jahre & $\begin{array}{l}\text { physiologisch } \\
\text { mit und ohne Resek- } \\
\text { tion des Brustkorbes }\end{array}$ \\
\hline Naderi et al. 1998 & 8 & C0-C3 & polysegmental & $\begin{array}{l}\text { 61,1 Jahre } \\
\text { (18-87Jahre) }\end{array}$ & $\begin{array}{l}\text { physiologisch, } \\
\text { pathologisch, } \\
\text { cable fixation }\end{array}$ \\
\hline Nägerl et al. 2009 & 9 & $\begin{array}{l}\text { L3-L4 } \\
\text { L4-L5 } \\
\text { T2-T3 }\end{array}$ & monosegmental & $\begin{array}{l}\text { 59,8Jahre +/- } \\
\text { 16,6Jahre }\end{array}$ & physiologisch \\
\hline Niosi et al. 2006 & 10 & L2-L5 & polysegmental & $\begin{array}{l}\text { 77,2 Jahre } \\
\text { (70-88 Jahre) }\end{array}$ & $\begin{array}{l}\text { physiologisch patho- } \\
\text { logisch mit } \\
\text { Implantatsystem } \\
\text { (Dynesys) }\end{array}$ \\
\hline Oxland et al. 1994 & 13 & $\begin{array}{l}\text { T11-L1 } \\
\text { T12-L2 }\end{array}$ & bisegmental & $\begin{array}{l}53 \text { Jahre } \\
\text { (19-70 Jahre) }\end{array}$ & $\begin{array}{l}\text { physiologisch, } \\
\text { pathologisch }\end{array}$ \\
\hline Rousseau et al. 2006a & 12 & L5-S1 & monosegmental & 45-64 Jahre & physiologisch \\
\hline Rousseau et al. 2006b & 12 & L5-S1 & monosegmental & $\begin{array}{l}\text { k. A. } \\
\text { (50-64 Jahre) }\end{array}$ & physiologisch \\
\hline Schulze et al. 2012 & $\begin{array}{l}12 \text { (Kalb- } \\
\text { präparate) }\end{array}$ & $\begin{array}{l}\text { L3-L4 } \\
\text { L2-L6 }\end{array}$ & $\begin{array}{l}\text { monosegmental } \\
\text { polysegmental }\end{array}$ & 12-24Wochen & physiologisch \\
\hline Seligman et al. 1984 & 47 & L4-L5 & monosegmental & $<60$ Jahre & $\begin{array}{l}\text { physiologisch } \\
\text { pathologisch }\end{array}$ \\
\hline Sengupta et al.2011 & 10 & L3-L5 & bisegmental & k. A. & k. A. \\
\hline Takigawa et al. 2010 & 14 & L4-L5 & monosegmental & $\begin{array}{l}\text { 80,4Jahre } \\
\text { (51-99 Jahre) }\end{array}$ & $\begin{array}{l}\text { physiologisch } \\
\text { Implantatsystem }\end{array}$ \\
\hline Wachowski et al. 2007 & 3 & C3-C4 & monosegmental & k. A. & $\begin{array}{l}\text { physiologisch } \\
\text { pathologisch }\end{array}$ \\
\hline
\end{tabular}




\begin{tabular}{|c|c|c|c|c|c|}
\hline $\begin{array}{l}\text { Wachowski et al. } \\
\text { 2009a }\end{array}$ & 8 & $\begin{array}{l}\text { C3-C4 } \\
\text { T6-T7 } \\
\text { T7-T8 } \\
\text { L3-L4 }\end{array}$ & monosegmental & $\begin{array}{l}66,6+/-4,8 \\
\text { Jahre }\end{array}$ & physiologisch \\
\hline $\begin{array}{l}\text { Wachowski et al. } \\
\text { 2009b }\end{array}$ & 8 & $\begin{array}{l}\text { L1-L2 } \\
\text { L3-L4 } \\
\text { L4-L5 }\end{array}$ & monosegmental & $\begin{array}{l}\text { 59,8 Jahre +/- } \\
\text { 16,6 Jahre }\end{array}$ & physiologisch \\
\hline $\begin{array}{l}\text { Wachowski et al. } \\
\text { 2010a }\end{array}$ & 7 & $\begin{array}{l}\text { T6-T7 } \\
\text { L3-L4 } \\
\text { L4-L5 }\end{array}$ & monosegmental & k. A. & physiologisch \\
\hline $\begin{array}{l}\text { Wachowski et al. } \\
\text { 2010b }\end{array}$ & 13 & $\begin{array}{l}\text { L1-L2 } \\
\text { L3-L4 } \\
\text { L4-L5 }\end{array}$ & monosegmental & $\begin{array}{l}\text { Medianwert: } \\
58 \text { Jahre } \\
\text { (45-86 Jahre) }\end{array}$ & $\begin{array}{l}\text { physiologisch } \\
\text { pathologisch }\end{array}$ \\
\hline Wachowski et al. 2013 & 8 & C3-C4 & monosegmental & $\begin{array}{l}\text { 68,7 Jahre +/- } \\
\text { 13,5 Jahre }\end{array}$ & $\begin{array}{l}\text { physiologisch } \\
\text { Implantatsystem }\end{array}$ \\
\hline $\begin{array}{l}\text { Zhu et al. } \\
2007\end{array}$ & 13 & L3-S1 & polysegmental & $\begin{array}{l}\text { 59 Jahre } \\
\text { (40-54 Jahre) }\end{array}$ & $\begin{array}{l}\text { physiologisch, } \\
\text { pathologisch, } \\
\text { Implantatsystem }\end{array}$ \\
\hline $\begin{array}{l}\text { Zhu et al. } \\
2012\end{array}$ & 6 & T7-S1 & polysegmental & $\begin{array}{l}66 \text { Jahre } \\
\text { (40-86 Jahre) }\end{array}$ & $\begin{array}{l}\text { physiologisch, } \\
\text { pathologisch, } \\
\text { Implantatsystem }\end{array}$ \\
\hline
\end{tabular}

\subsection{Messapparaturen}

Die Testapparaturen beinhalteten eine Messeinrichtung zur Aufzeichnung der erfolgten Bewegung der Segmente. Aus den Messdaten konnten mittels Computersoftware biomechanische und kinematische Parameter der Bewegung ermittelt werden (s. Kap. 3.6 und 3.7). Prinzipiell lassen sich die von den Autoren verwendeten Messtechniken in verschiedene Gruppen einteilen. So wurde die Bewegung über Röntgenbilder, optisch, mechanisch oder mittels Ultraschallwellen erfasst (s.u.). In Tabelle 4 findet sich ein Vermerk für das von den Autoren jeweils genutzte System. Es war entsprechend der verwendeten Messapparatur möglich, die Bewegung zwei- oder dreidimensional zu erfassen (Tab. 4). Ein weiterer Unterschied bestand in der örtlichen und zeitlichen Auflösungsfähigkeit der verwendeten Messapparatur (Tab. 4):

Unter der örtlichen Auflösungsfähigkeit der Bewegung wird die Genauigkeit verstanden, mit der die Lokalisation der Marker aufgezeichnet werden konnte bzw. die kleinste Bewegungsstrecke des Versuchsobjektes, die von der Messapparatur erfasst werden konnte. Hierzu fanden sich unterschiedliche Angaben (Tab. 4). 
Unter der zeitlichen Auflösungsfähigkeit wird das Zeitintervall bzw. die Frequenz verstanden, zwischen denen jeweils die Positionen des Versuchsobjektes während der Bewegung bestimmt wurden. Von den Autoren wurde dabei nicht angegeben, ob jeweils die höchste Frequenz des gewählten Messsystems ausgeschöpft wurde. Die Frequenz in der die Lokalisation der Präparate aufgezeichnet wurde, variierte dabei von $2 \mathrm{~Hz}$ (Brasiliense et al. 2011) bis $60 \mathrm{~Hz}$ (Cusick et al. 1988). Wachowski et al. gaben an 800 Positionsbestimmungen pro ROM bei angelegtem axialen Rotationsmoment in lumbalen Segmenten erheben zu können (Wachowski et al. 2010). Einige Autoren machten keine Angaben bezüglich der zeitlichen Auflösungsfähigkeit der Messapparatur (Tab. 4).

\subsubsection{Röntgenbildanalysen}

Bei Röntgenbildanalysen ließen sich über festgelegte Referenzmarker innerhalb des sich bewegenden Segmentes Rückschlüsse auf die Position der Wirbel ziehen, indem die Position der Marker vor und nach der Bewegung über Röntgenbilder bestimmt wurde (Seligman et al. 1984, Molnar et al. 2006).

\subsubsection{Optische Verfahren}

Auch bei optischen Verfahren ließen sich über festgelegte Referenzmarker innerhalb des sich bewegenden Segmentes Rückschlüsse auf die Position der Wirbel ziehen. Als Referenzmarker dienten dabei Infrarotlicht-emittierende Dioden (z. B. Bozkus et al. 2010) oder zuvor an das Präparat angebrachte Marker (z. B. Cusick et al. 1988). Die Bewegung der Referenzpunkte wurde dann durch Videokameras, Fotokameras oder optoelektronische Systeme aufgezeichnet (Tab. 4). Bei optoelektronischen Systemen, welche von den meisten Autoren verwendet wurden (Tab. 4), sendeten mehrere an den Testobjekten angebrachte Dioden Infrarotlicht aus. Ein optoelektronisches System empfing das Infrarotlicht und konnte so über die Lokalisation der Infrarotlichter auf die jeweilige Position der sich bewegenden Wirbel schließen (Dickman et al. 1996, Broc et al. 1997, Grassmann et al. 1998, Naderi et al. 1998, Chen et al. 2001, Cripton et al. 2001, Crawford et al. 2002, Detwiler et al. 2003, Haberl et al. 2004, Kotani et al. 2006, Niosi et al. 2006, Zhu et al. 2007, Bozkus et al. 2010, Lazaro et al. 2010, Brasiliense et al. 2011, Sengupta et al. 2011, Zhu et al. 2012, Schulze et al. 2012). 


\subsubsection{Mechanische Verfahren}

Bei mechanischen Messverfahren erfolgte die Messung der Bewegung des Versuchsobjektes über Messtaster, die direkt und starr mit dem Versuchsobjekt verbunden waren, entsprechend sind auch keine Referenzpunkte verwendet worden.

Mansour et al. (2004) erfassten die Bewegung über mechanische Taster. Insgesamt sechs Lagesensoren berührten in einer 3-2-1 Anordnung senkrecht drei Glasplatten, die in den jeweils drei Ebenen des Koordinatensystems angeordnet waren und fest mit dem zu untersuchenden Präparat in Verbindung standen. Bei Bewegung des zu testenden Segmentes bewegten sich entsprechend die Glasplatten, wodurch die Position des Segmentes durch die Messtaster bestimmt werden konnte (Mansour et al. 2004). Dieses Verfahren wurde auch in anderen Studien angewendet (Wachowski et al. 2007, Nägerl et al. 2009, Wachowski et al. 2007, Wachowski et al. 2009a, Wachowski et al. 2009b, Wachowski et al. 2010, Wachowski et al. 2010b, Wachowski et al. 2013).

\subsubsection{Messung über Ultraschall}

Kettler et al. verwendeten ein System bei dem drei Ultraschall Transmitter am oberen Wirbel und drei bis vier Ultraschall Receiver am unteren Wirbel angebracht waren. Der sich bei der Bewegung ändernde Zeitabstand zwischen Senden und Empfang des Ultraschalls diente zur Auflösung der Position der Wirbel (Kettler et al. 2004).

Tabelle 4 gibt einen Überblick über die in den jeweiligen Studien verwendeten Messvorgänge zur Bewegungsaufzeichnung.

Tab. 4 Verwendete Messapparaturen in den untersuchten Studien

\begin{tabular}{|l|l|l|l|l|}
\hline & $\begin{array}{l}\text { genutztes Messsys- } \\
\text { tem zur Aufzeich- } \\
\text { nung }\end{array}$ & $\begin{array}{l}\text { Örtliche Auflö- } \\
\text { sungs- } \\
\text { möglichkeit } \\
\text { des genutzen } \\
\text { Messsystems }\end{array}$ & $\begin{array}{l}\text { zeitliche Auf- } \\
\text { lösungs- } \\
\text { möglichkeit } \\
\text { des genutz- } \\
\text { ten Messsys- } \\
\text { tems }\end{array}$ & $\begin{array}{l}\text { erfassbare } \\
\text { Dimensionen }\end{array}$ \\
\hline $\begin{array}{l}\text { Bozkus et al. 2010 } \\
\text { erste Testappara- } \\
\text { tur }\end{array}$ & $\begin{array}{l}\text { optisch über } \\
\text { „Optotrak 3020 opto- } \\
\text { electronic camera } \\
\text { system“ }\end{array}$ & $\begin{array}{l}0,1 \mathrm{~mm} \\
\text { (pers. Mitteilung: } \\
\text { Crawford 2014) }\end{array}$ & k. A. & 3-dimensional \\
\hline Bozkus et al. 2010 & $\begin{array}{l}\text { optisch über } \\
\text { "Optotrak 3020 opto- } \\
\text { electronic camera } \\
\text { system“ }\end{array}$ & $\begin{array}{l}0,1 \mathrm{~mm} \\
\text { (pers. Mitteilung: } \\
\text { Crawford 2014) }\end{array}$ & k. A. & 3-dimensional \\
\hline Brasiliense et al. & $\begin{array}{l}\text { optisch über } \\
\text { „Optotrak 3020 opto- }\end{array}$ & $0,1 \mathrm{~mm}$ & $2 \mathrm{~Hz}$ & 3-dimensional \\
\hline
\end{tabular}




\begin{tabular}{|c|c|c|c|c|}
\hline 2011 & $\begin{array}{l}\text { electronic camera } \\
\text { system“ }\end{array}$ & $\begin{array}{l}\text { (pers. Mitteilung: } \\
\text { Crawford 2014) }\end{array}$ & & \\
\hline Broc et al. 1997 & $\begin{array}{l}\text { optisch über } \\
\text { „Optotrak opto- } \\
\text { electronic camera } \\
\text { system“ }\end{array}$ & $\begin{array}{l}0,1 \mathrm{~mm} \\
\text { (pers. Mitteilung: } \\
\text { Crawford 2014) }\end{array}$ & k. A. & 3-dimensional \\
\hline Cagli et al. 2004 & $\begin{array}{l}\text { optisch über } \\
\text { „Optotrak opto- } \\
\text { electronic camera } \\
\text { system“ }\end{array}$ & $\begin{array}{l}0,1 \mathrm{~mm} \\
\text { (pers. Mitteilung: } \\
\text { Crawford 2014) }\end{array}$ & $28 \mathrm{~Hz}$ & 3-dimensional \\
\hline Chen et al. 2001 & $\begin{array}{l}\text { optisch über } \\
\text { "Optotrak } 3020 \text { opto- } \\
\text { electronic camera } \\
\text { system“ }\end{array}$ & $\begin{array}{l}0,1 \mathrm{~mm} \\
\text { (pers. Mitteilung: } \\
\text { Crawford 2014) }\end{array}$ & k. A. & 3-dimensional \\
\hline $\begin{array}{l}\text { Crawford et al. } \\
2002\end{array}$ & $\begin{array}{l}\text { optisch über } \\
\text { „Optotrak } 3020 \text { opto- } \\
\text { electronic camera } \\
\text { system“ }\end{array}$ & $\begin{array}{l}0,1 \mathrm{~mm} \\
\text { (pers. Mitteilung: } \\
\text { Crawford 2014) }\end{array}$ & $42 \mathrm{~Hz}$ & 3-dimensional \\
\hline Cripton et al. 2001 & $\begin{array}{l}\text { optisch über } \\
\text { „Optotrak } 3020 \text { opto- } \\
\text { electronic camera } \\
\text { system“ }\end{array}$ & $0,15 \mathrm{~mm}$ & $10 \mathrm{~Hz}$ & 3-dimensional \\
\hline Cusick et al. 1988 & $\begin{array}{l}\text { optisch über Fotoka- } \\
\text { mera }\end{array}$ & k. A. & $60 \mathrm{~Hz}$ & 2-dimensional \\
\hline $\begin{array}{l}\text { Detwiler et al. } \\
2003\end{array}$ & $\begin{array}{l}\text { optisch über } \\
\text { "Optotrak } 3020 \text { opto- } \\
\text { electronic camera } \\
\text { system“ }\end{array}$ & $\begin{array}{l}0,1 \mathrm{~mm} \\
\text { (pers. Mitteilung: } \\
\text { Crawford 2014) }\end{array}$ & k. A. & 3-dimensional \\
\hline $\begin{array}{l}\text { Dickman et al. } \\
1996\end{array}$ & $\begin{array}{l}\text { optisch über } \\
\text { „Optotrak motion } \\
\text { analyses system“ }\end{array}$ & $\begin{array}{l}0,1 \mathrm{~mm} \\
\text { (pers. Mitteilung: } \\
\text { Crawford 2014) }\end{array}$ & k. A. & 3-dimensional \\
\hline $\begin{array}{l}\text { Dugailly et al. } \\
2010\end{array}$ & $\begin{array}{l}\text { optisch über } \\
\text { 3d-digitizer } \\
\text { (Faro B06/Rev18) }\end{array}$ & $\begin{array}{l}\text { max. Standard- } \\
\text { fehler bei Digita- } \\
\text { lisierung der } \\
\text { Markerpos. } \\
+/-0,47 \mathrm{~mm} \\
\\
\text { error in marker- } \\
\text { digitizing } 0,125 \\
\mathrm{~mm}-0,3 \mathrm{~mm}\end{array}$ & k. A. & 3-dimensional \\
\hline $\begin{array}{l}\text { Dugailly et al. } \\
2011\end{array}$ & $\begin{array}{l}\text { optisch über } \\
\text { 3d-digitizer } \\
\text { (k. A.) } \\
\text { CT-Bilder }\end{array}$ & $\begin{array}{l}\text { k. A. Unter- } \\
\text { schied der Loa- } \\
\text { kalisation der } \\
\text { Marker zwi- } \\
\text { schen 3d- digiti- } \\
\text { zer und CT- } \\
\text { Bilder }=0,9 \mathrm{~mm}\end{array}$ & k. A. & 3-dimensional \\
\hline $\begin{array}{l}\text { Dugailly et al. } \\
2013\end{array}$ & $\begin{array}{l}\text { optisch über 3d- } \\
\text { digitizer } \\
\text { CT-Bilder }\end{array}$ & $\begin{array}{l}\text { Unterschied der } \\
\text { Loakalisation } \\
\text { der Marker } \\
\text { zwischen } 3 \mathrm{~d}- \\
\text { digitizer und CT- } \\
\text { Bilder }=<1 \mathrm{~mm}\end{array}$ & k. A. & 3-dimensional \\
\hline
\end{tabular}




\begin{tabular}{|c|c|c|c|c|}
\hline $\begin{array}{l}\text { Grassmann et al. } \\
1998 \\
\text { Erste Testappara- } \\
\text { tur }\end{array}$ & k. A. & k. A. & k. A. & k. A. \\
\hline $\begin{array}{l}\text { Grassmann et al. } \\
1998 \text { zweite Test- } \\
\text { apparatur }\end{array}$ & $\begin{array}{l}\text { optisch über } \\
\text { "Optotrak } 3020 \text { opto- } \\
\text { electronic camera } \\
\text { system" }\end{array}$ & $\begin{array}{l}0.2 \mathrm{~mm} \text { für } \\
\text { Translationen } \\
\text { und } 0.1^{\circ} \text { für } \\
\text { Rotationen } \\
\text { (pers.Mitteilung: } \\
\text { Oxland 2014) }\end{array}$ & $\begin{array}{l}800 \mathrm{~Hz} \\
\text { (pers. Mittei- } \\
\text { lung: Oxland } \\
\text { 2014) }\end{array}$ & 3-dimensional \\
\hline Haberl et al. 2004 & $\begin{array}{l}\text { optisch über } \\
\text { "Optotrak motion } \\
\text { analyses system" }\end{array}$ & k. A. & k. A. & 3-dimensional \\
\hline Haher et al. 1991 & optisch Fotokamera & k. A. & k. A. & 2-dimensional \\
\hline Haher et al. 1992 & optisch Fotokamera & k. A. & k. A. & 2-dimensional \\
\hline Kettler et al.2004 & Ultraschallsystem & $0,1 \mathrm{~mm}$ & $20 \mathrm{~Hz}$ & 3-dimensional \\
\hline $\begin{array}{l}\text { Lazaro et al. } 2010 \\
\star \star \text { erste Testappa- } \\
\text { ratur }\end{array}$ & $\begin{array}{l}\text { optisch über } \\
\text { „Optotrak } 3020 \text { opto- } \\
\text { electronic camera } \\
\text { system“ }\end{array}$ & $\begin{array}{l}\text { 0,1 mm } \\
\text { (pers. Mitteilung: } \\
\text { Crawford 2014) }\end{array}$ & k. A. & 3-dimensional \\
\hline $\begin{array}{l}\text { Lazaro et al. } 2010 \\
\text { zweite Testappa- } \\
\text { ratur }\end{array}$ & $\begin{array}{l}\text { optisch über } \\
\text { „Optotrak } 3020 \text { opto- } \\
\text { electronic camera } \\
\text { system“ }\end{array}$ & $\begin{array}{l}0,1 \mathrm{~mm} \\
\text { (pers. Mitteilung: } \\
\text { Crawford 2014) }\end{array}$ & k. A. & 3-dimensional \\
\hline $\begin{array}{l}\text { Mansour et al. } \\
2004\end{array}$ & $\begin{array}{l}\text { mechanisch über } \\
\text { Messtaster }\end{array}$ & $\begin{array}{l}0.01-2.4 \mu \mathrm{m} \text { für } \\
\text { Translationen } \\
3 \mu \text { deg für Rota- } \\
\text { tionen }\end{array}$ & $\begin{array}{l}\text { kontinuierlich } \\
600-800 \text { Meß- } \\
\text { werte/ Zyklus }\end{array}$ & 3-dimensional \\
\hline McNally et al. 2012 & optisch über Kamera & k. A. & $50 \mathrm{~Hz}$ & 2-dimensional \\
\hline Molnar et al. 2006 & Röntgenbilder & k. A. & k. A. & k. A. \\
\hline Naderi et al. 1998 & $\begin{array}{l}\text { optisch über } \\
\text { „Optotrak } 3020 \text { opto- } \\
\text { electronic camera } \\
\text { system“ }\end{array}$ & $\begin{array}{l}\text { 0,1 mm } \\
\text { (pers. Mitteilung: } \\
\text { Crawford 2014) }\end{array}$ & k. A. & 3-dimensional \\
\hline Nägerl et al. 2009 & $\begin{array}{l}\text { mechanisch über } \\
\text { Messtaster }\end{array}$ & $\begin{array}{l}0,01 \mu \mathrm{m}-2,4 \mu \mathrm{m} \\
0,5 \mu \mathrm{m} \text { für } \\
\text { Translationen } \\
0,5 \text { mdeg für } \\
\text { Rotationen } \\
\text { für das gesamte } \\
\text { Koordinatensys- } \\
\text { tem }\end{array}$ & $<400 / \mathrm{ROM}$ & 3-dimensional \\
\hline Niosi et al. 2006 & $\begin{array}{l}\text { optisch über } \\
\text { "Optotrak } 3020 \text { opto- } \\
\text { electronic camera } \\
\text { system“ }\end{array}$ & $\begin{array}{l}0.2 \mathrm{~mm} \text { für } \\
\text { Translationen } \\
\text { und } 0.1^{\circ} \text { für } \\
\text { Rotationen } \\
\text { pers.Mitteilung }\end{array}$ & $20 \mathrm{~Hz}$ & 3-dimensional \\
\hline
\end{tabular}




\begin{tabular}{|c|c|c|c|c|}
\hline Oxland et al. 1994 & $\begin{array}{l}\text { optisch Stereofoto- } \\
\text { grafie }\end{array}$ & k. A. & k. A. & 3-dimensional \\
\hline $\begin{array}{l}\text { Rousseau et al. } \\
2006 a\end{array}$ & $\begin{array}{l}\text { optisch über Kame- } \\
\text { rasystem }\end{array}$ & $+/-0,25 \mathrm{~mm}$ & k. A. & 3-dimensional \\
\hline $\begin{array}{l}\text { Rousseau et al. } \\
\text { 2006b }\end{array}$ & $\begin{array}{l}\text { optisch über Kame- } \\
\text { rasystem }\end{array}$ & $+/-0,25 \mathrm{~mm}$ & k. A. & 3-dimensional \\
\hline Schulze et al. 2012 & $\begin{array}{l}\text { optisch über „Opto- } \\
\text { trak-Certus opto- } \\
\text { electronic camera } \\
\text { system“ }\end{array}$ & $\begin{array}{l}0,001+/-0.034 \\
\text { mm für Transla- } \\
\text { tionen und } \\
0,075+/-0,12^{\circ} \\
\text { für Rotationen }\end{array}$ & k. A. & 3-dimensional \\
\hline $\begin{array}{l}\text { Seligman et al. } \\
1984\end{array}$ & Röntgenbilder & k. A. & k. A. & 2-dimenisonal \\
\hline $\begin{array}{l}\text { Sengupta et al. } \\
2011\end{array}$ & $\begin{array}{l}\text { optisch über } \\
\text { „Optotrak } 3020 \text { opto- } \\
\text { electronic camera } \\
\text { system” }\end{array}$ & k. A. & $\begin{array}{l}\text { "kontinuierli- } \\
\text { che Aufzeich- } \\
\text { nung der } \\
\text { Bewegung" }\end{array}$ & 3-dimensional \\
\hline $\begin{array}{l}\text { Takigawa et al. } \\
2010\end{array}$ & $\begin{array}{l}\text { optisch über Kame- } \\
\text { rasystem }\end{array}$ & k. A. & k. A. & 3-dimensional \\
\hline $\begin{array}{l}\text { Wachowski et al. } \\
2007\end{array}$ & $\begin{array}{l}\text { mechanisch über } \\
\text { Messtaster }\end{array}$ & $\begin{array}{l}2,4 \mu \mathrm{m} \text { für } \\
\text { Translation } \\
\text { 1mdeg für Rota- } \\
\text { tionen }\end{array}$ & Je $0,01^{\circ}$ & 3-dimensional \\
\hline $\begin{array}{l}\text { Wachowski et al. } \\
\text { 2009a }\end{array}$ & $\begin{array}{l}\text { mechanisch über } \\
\text { Messtaster }\end{array}$ & $\begin{array}{l}0,01 \mu \mathrm{m} \text { der } \\
\text { einzelnen } \\
\text { Messtaster } \\
0,5 \mu \mathrm{m} \text { für } \\
\text { Translation und } \\
0,001^{\circ} \text { für Rota- } \\
\text { tionen } \\
\text { Für das gesam- } \\
\text { te Koordinaten- } \\
\text { system }\end{array}$ & 400/ ROM & 3-dimensional \\
\hline $\begin{array}{l}\text { Wachowski et al. } \\
2009 \text { b }\end{array}$ & $\begin{array}{l}\text { mechanisch über } \\
\text { Messtaster }\end{array}$ & $\begin{array}{l}0,5 \mu \mathrm{m} \text { für } \\
\text { Translationen } \\
\text { des gesamten } \\
\text { Koordinaten- } \\
\text { systems }\end{array}$ & 400/ ROM & 3-dimensional \\
\hline $\begin{array}{l}\text { Wachowski et al. } \\
2010 a\end{array}$ & $\begin{array}{l}\text { mechanisch über } \\
\text { Messtaster }\end{array}$ & $\begin{array}{l}0,01 \mu \mathrm{m}-2,4 \mu \mathrm{m} \\
\text { der einzelnen } \\
\text { Messtaster } \\
0,5 \mu \mathrm{m} \text { für } \\
\text { Translation und } \\
0,001^{\circ} \text { für Rota- } \\
\text { tionen } \\
\text { Für das gesam- } \\
\text { te Koordinaten- } \\
\text { system }\end{array}$ & k. A. & 3-dimensional \\
\hline $\begin{array}{l}\text { Wachowski et al. } \\
\text { 2010b }\end{array}$ & $\begin{array}{l}\text { mechanisch über } \\
\text { Messtaster }\end{array}$ & $0,01-2,4 \mu \mathrm{m}$ & $\begin{array}{l}800 / \mathrm{ROM} \\
\text { In Interval- } \\
\text { len }<0,1^{\circ}\end{array}$ & 3-dimensional \\
\hline
\end{tabular}




\begin{tabular}{|c|c|c|c|c|}
\hline $\begin{array}{l}\text { Wachowski et al. } \\
2013\end{array}$ & $\begin{array}{l}\text { mechanisch über } \\
\text { Messtaster }\end{array}$ & $\begin{array}{l}<1 \mu \mathrm{m} \text { Transla- } \\
\text { tionen } \\
<1 \text { mdeg Rotati- } \\
\text { onen } \\
\text { Für das gesam- } \\
\text { te Koordinaten- } \\
\text { system }\end{array}$ & 400/ ROM & 3-dimensional \\
\hline $\begin{array}{l}\text { Zhu et al. } \\
2007\end{array}$ & $\begin{array}{l}\text { optisch über „Opto- } \\
\text { trak } 3020 \\
\text { Optoelectronic motion } \\
\text { analysis system“ }\end{array}$ & $\begin{array}{l}0,1-0,15 \mathrm{~mm} \\
\text { (pers. Mitteilung } \\
\text { Oxland 2014) }\end{array}$ & $20 \mathrm{~Hz}$ & 3-dimensional \\
\hline $\begin{array}{l}\text { Zhu et al. } \\
2012\end{array}$ & $\begin{array}{l}\text { optisch über "Opto- } \\
\text { trak } 3020 \\
\text { Optoelectronic motion } \\
\text { analysis system" }\end{array}$ & $\begin{array}{l}0,1-0,15 \mathrm{~mm} \\
\text { (pers. Mitteilung } \\
\text { Oxland 2014) }\end{array}$ & $\begin{array}{l}800 \mathrm{~Hz} \text { (pers. } \\
\text { Mitteilung: } \\
\text { Oxland 2014) }\end{array}$ & 3-dimensional \\
\hline
\end{tabular}

*für den Versuch, in dem eine Rotationsachse berechnet wurde 


\subsection{Angaben zu Rotations-/Schraubachsen der untersuchten Studien}

Gemäß der Ein- und Ausschlusskriterien der vorliegenden Studie wurde von allen Autoren der zur Analyse herangezogenen Studien eine Aussage bezüglich einer Rotations-/Schraubachse gemacht. Wie bereits im Kapitel 1.5.3 angeführt, finden sich in der Literatur verschiedene Theorien, wie eine Rotations-/Schraubachse zu betrachten sei. So gingen einige Autoren von einer dreidimensionalen helical axis of motion (HAM) aus (s. Tab. 5.), um die die Translation und Rotation während der erfolgten Bewegung geschehen solle. Zur Berechnung einer HAM wurden zwei Positionen des Segmentes während der erfolgten Bewegung benötigt. Dabei handelte es sich um Anfangs- und Endpunkt der Bewegung. Das Bewegungsintervall, das zur Berechnung der Achse genutzt wurde, umfasste somit das gesamte ROM (Niosi et al. 2006, Zhu et al. 2007, Zhu et al. 2012). Andere Autoren berechneten die HAM für kleinere Intervalle (s. Tab. 5). So teilten Cripton et al. das ROM in 0,5 - Intervalle und berechneten für jedes Intervall eine HAM (Cripton et al. 2000). Andere Autoren teilten das ROM ebenfalls in Intervalle und nannten die berechneten Achsen jeweils finite helical axis (FHA) (Kettler et al. 2004, Schulze et al. 2012). Crawford et al. gaben an, die Lage der Achse für jeweils 0,5 - Intervalle berechnen zu können und verwendeten dafür die Bezeichnung finite axis of rotation, helical axis of motion oder axis of rotation (Crawford et al. 2002). Andere Autoren waren in der Lage, das ROM in kleinere Intervalle zu unterteilen. So gaben Wachowski et al. an, das ROM in 800 Intervalle geteilt zu haben und für jedes Intervall eine instantaneous helical axis (IHA) angeben zu können (Wachowski et al. 2010). Mansour et al. gaben an, die Lokalisation einer IHA für Rotationsintervalle von $0,02^{\circ}$ berechnen zu können (Mansour et al. 2004).

Wird die Bewegung um eine Achse nur in Bezug auf die Rotation und nicht die Translation betrachtet, verwendeten manche Autoren die Bezeichnung instantaneous axis of rotation (Rousseau et al. 2006a, Bozkus et al. 2010). Sengupta et al. definierten die IAR hingegen als Rotationsachse um die sowohl Rotation als auch Translation stattfinden könne (Sengupta et al. 2011).

Auch bezüglich der IAR unterschieden sich die Autoren bezüglich der Intervalle, für die eine Rotationsachse angegeben werden konnte (s. Tab. 5).

Detwiler et al. (2003) berechneten die Lokalisation der axis of rotation oder finite axis of rotation vom oberen Bereich der neutralen Zone (NZ) bis zum vollen ROM und erhielten so 2 Lokalisationsangaben innerhalb des gesamten ROM. In diesem Bereich der elastischen Zone (EZ), wurde der für die Bestimmung der Rotationsachse 
relevante Bereich angenommen, da hier größere Veränderungen nach Laminektomie im Vergleich zu Testdurchläufen ohne Laminektomie erkennbar gewesen sein sollten (Detwiler et al. 2003).

Neben den genannten Achsen fanden sich weitere Bezeichnungen wie helical axis (HA) (Dugailly et al. 2013), rotational axis (Molnar et al. 2006) oder axis of rotation (AOR) (Brasiliense et al. 2011) oder finite axis of rotation (Detwiler et al. 2003) (Tab.5). Brasiliense bezeichneten die AOR, für die eine Lokalisation innerhalb des gesamten ROM angegeben wurde, zugleich auch als finite helical axis of motion (Brasiliense et al. 2011).

Zusätzlich zu den Angaben zur Lokalisation machten einige Autoren auch Aussagen zur Ausrichtung der Achse (s. Tab.5) im dreidimensionalem Raum. Dies konnte dabei in Form von Richtungsvektoren (z. B. Mansour et al. 2004, Wachowski et al. 2009a und andere), Gradzahlen in Bezug auf die Neigung der Rotationsachse zu den Achsen des Koordinatensystems (z. B. Oxland et al. 1994, Dugailly et al. 2010 und andere) bzw. aus Abbildungen, die den Verlauf der Rotationsachse im dreidimensionalen Raum darstellten (z. B. Haberl et al. 2004, Kettler et al. 2004 und andere) ersichtlich werden (s. Tab.5). Konkrete Beschreibungen bezüglich der Angaben zur Ausrichtung der Rotations-/Schraubachsen für zervikale und lumbale Segmente finden sich in Kapitel 3.7.

In einigen Fällen wurden Validierungstests durchgeführt, mit denen festgestellt werden konnte, wie genau die Position der Rotations-/Schraubachsen angegeben werden konnte. Rousseau et al. validierten ihr Testprotokoll mit Hilfe eines Scharniergelenkes. Dabei gaben die Autoren an, bei einer Rotation von $3^{\circ}$ eine Abweichung der errechneten Lage der IAR von $4 \mathrm{~mm}$, im Vergleich zur tatsächlichen, bekannten Rotationsachse des Scharniergelenkes festzustellen (Rousseau et al. 2006a). Kettler et al. gaben an, dass die berechnete FHA mit einer Genauigkeit von +/- 2,5 mm angegeben werden konnte und im Vergleich zur Scharnierachse $+/-1,5^{\circ}$ geneigt war (Kettler et al. 2004) (s. Tab 5). Zhu et al. konnten nach eigenen Angaben, die Position der HAM mit einer Genauigkeit von $5 \mathrm{~mm}$ angeben für den Fall, dass das Segment mehr als $1^{\circ}$ Rotation vollzog (Zhu et al. 2007). Andere Autoren gaben nach Durchführung eines Validierungstests eine Abweichung der Lokalisation der Rotationsachse von der tatsächlichen Position von +/-1 mm an (Mansour et al. 2004). 
Eine weitere Unterscheidung fand sich in der angelegten Bewegungsrichtung für die eine Rotationsachse ermittelt wurde.

Einige Autoren errechneten die Position der Rotationsachsen nicht für alle Richtungen, für die ein Drehmoment angelegt wurde (vgl. Kap. 3.2) (Tab. 1, S. 33), da hier Bewegungen außerhalb der erfolgten Hauptbewegungsebene, also Bewegungen im Sinne der coupled motion zu groß seien (Naderi et al. 1998, Cagli et al. 2004). Dies könnte nach Angaben der Autoren zu Ungenauigkeiten der Messergebnisse führen.

Die aus dem jeweils angelegten Drehmoment resultierenden Bewegungsrichtungen, für die eine Rotations-/Schraubachse berechnet wurde, sind in Tab. 5 dargestellt.

Tab.5 Übersicht über die ermittelten Rotations-/Schraubachsen in den untersuchten Studien.

\begin{tabular}{|c|c|c|c|c|c|}
\hline & $\begin{array}{l}\text { Name der Rota- } \\
\text { tionsachse nach } \\
\text { Angaben der } \\
\text { Autoren }\end{array}$ & $\begin{array}{l}\text { Anzahl berech- } \\
\text { neter Achsen } \\
\text { bzw. Intervalle } \\
\text { pro Lokalisati- } \\
\text { onsangabe }\end{array}$ & $\begin{array}{l}\text { Angaben zu } \\
\text { Lage und Aus- } \\
\text { richtung der } \\
\text { Achse }\end{array}$ & $\begin{array}{l}\text { Genauigkeit } \\
\text { der Angabe der } \\
\text { Lokalisation } \\
\text { der Achse nach } \\
\text { Validierungs- } \\
\text { protokoll }\end{array}$ & $\begin{array}{l}\text { Angelegte } \\
\text { Drehmoment- } \\
\text { richtungen für } \\
\text { die eine Achse } \\
\text { angegeben } \\
\text { wurde }\end{array}$ \\
\hline Bozkus et al. 2010 & IAR & $\begin{array}{l}0,5^{\circ} \text { - Intervalle } \\
\text { Angabe der } \\
\text { Lokalisation } \\
\text { erfolgt aber als } \\
\text { mean helical } \\
\text { axis } \rightarrow 1 \mathrm{IAR} / \\
\text { ROM }\end{array}$ & nein & k. A. & Fl-Ex \\
\hline Brasiliense et al. 2011 & $\begin{array}{l}\text { axis of rotation } \\
\text { (AOR) oder finite } \\
\text { helical axis }\end{array}$ & 1/ROM & nein & k. A. & FL-Ex \\
\hline Broc et al. 1997 & IAR bzw. FHA & $0,2^{\circ}$ & nein & k. A. & FI-Ex, AR, LB \\
\hline Cagli et al. 2004 & $\begin{array}{l}\text { finite axis of rota- } \\
\text { tion } \\
\text { axis of rotation } \\
\text { helical axis of } \\
\text { motion } \\
\text { instantaneous } \\
\text { axis of rotation }\end{array}$ & $\begin{array}{l}0,5^{\circ} \text { - Intervalle } \\
\text { Lokalisationen } \\
\text { werden als } \\
\text { Durchschnitts- } \\
\text { position ange- } \\
\text { geben }\end{array}$ & nein & k. A. & Fl-Ex \\
\hline Chen et al. 2001 & IAR & $\begin{array}{l}0,2^{\circ} \\
\text { Lokalisationen } \\
\text { werden als } \\
\text { Durchschnitts- } \\
\text { position ange- } \\
\text { geben }\end{array}$ & nein & k. A. & Fl-Ex \\
\hline
\end{tabular}




\begin{tabular}{|c|c|c|c|c|c|}
\hline Crawford et al. 2002 & $\begin{array}{l}\text { finite axis of rota- } \\
\text { tion }\end{array}$ & $\begin{array}{l}0,5^{\circ} \\
\text { Lokalisationen } \\
\text { werden als } \\
\text { Durchschnitts- } \\
\text { position ange- } \\
\text { geben }\end{array}$ & ja & nein & FI-Ex \\
\hline Cripton et al. 2001 & HAM & $\begin{array}{l}\text { 0,5-Intervalle } \\
6-12 \mathrm{HAM} / \mathrm{Be}- \\
\text { wegung }\end{array}$ & ja & $\begin{array}{l}\text { k. A. } \\
\text { (Bei Projektion } \\
\text { der Mess- } \\
\text { ergebnisse auf } \\
\text { CT-Bilder ent- } \\
\text { steht ein Regist- } \\
\text { rierungs-fehler } \\
\text { von }<1 \mathrm{~mm} \text { ) }\end{array}$ & AR nach rechts \\
\hline Cusick et al. 1988 & IAR & k. A. & nein & k. A. & $\mathrm{FI}$ \\
\hline Detwiler et al. 2003 & axis of rotation & 2/ ROM & nein & k. A. & FI-Ex \\
\hline Dickman et al. 1996 & IAR & $1^{\circ}$-Intervalle & nein & k. A. & $\mathrm{AR}$ \\
\hline Dugailly et al. 2009 & HA & 4/ ROM & ja & k. A. & AR, FI-Ex \\
\hline Dugailly et al. 2011 & $\mathrm{HA}$ & 4/ ROM & ja & $\begin{array}{l}1 \mathrm{~mm} \text { Fehler in } \\
\text { Lokalisation der } \\
\mathrm{HA}\end{array}$ & AR, FI-Ex \\
\hline Dugailly et al. 2013 & FHA & 4/ ROM & ja & k. A. & AR, FI-Ex \\
\hline $\begin{array}{l}\text { Grassmann et al.1998 } \\
\text { „unconstrained app.” }\end{array}$ & HAM & 8/ ROM & ja & k. A. & AR \\
\hline Haberl et al. 2004 & HAM & $5 / 1 / 2$ ROM & ja & k. A. & $\begin{array}{l}\text { AR unter kombi- } \\
\text { nierter FI-Ex }\end{array}$ \\
\hline Haher et al. 1991 & IAR & 2/ ROM , & nein & k. A. & FI-Ex \\
\hline Haher et al. 1992 & IAR & 4/ ROM & nein & k. A. & $\mathrm{AR}$ \\
\hline Kettler et al. 2004 & FHA & $<0,1^{\circ}$ - Intervalle & ja & $\begin{array}{l}\text { Lokalisation } \\
\text { FHA:+/2,5 mm } \\
\text { Orientierung } \\
\text { FHA:+/- } 1,5^{\circ}\end{array}$ & FI-Ex, AR, LB \\
\hline Lazaro et al. 2010 ** & IAR & $\begin{array}{l}0,5^{\circ} \text { - Intervalle } \\
\text { Lokalisationen } \\
\text { werden aber als } \\
\text { Durchschnitts- } \\
\text { position ange- } \\
\text { geben }\end{array}$ & nein & k. A. & FI-Ex \\
\hline Mansour et al. 2004 & IHA & $\begin{array}{l}\text { kontinuierlich, } \\
0,02^{\circ} \text { - Intervalle }\end{array}$ & ja & $+/-1 \mathrm{~mm}$ & AR \\
\hline McNally et al. 2012 & IAR & k. A. & nein & $+/-1,5 \mathrm{~mm}$ & $\mathrm{FI}$ \\
\hline Molnar et al. 2006 & rotational axis & k. A. & k. A. & k. A. & AR \\
\hline
\end{tabular}




\begin{tabular}{|c|c|c|c|c|c|}
\hline Naderi et al. 1998 & IAR & $\begin{array}{l}0,2^{\circ} \\
\text { Lokalisationen } \\
\text { werden aber als } \\
\text { Durchschnitts- } \\
\text { position ange- } \\
\text { geben }\end{array}$ & nein & k. A. & $\begin{array}{l}\text { AR } \\
\text { FI-Ex }\end{array}$ \\
\hline Nägerl et al. 2009 & $\mathrm{IHA}$ & $400 / \mathrm{ROM}$ & ja & k. A. & FI-Ex \\
\hline Niosi et al. 2006 & HAM & 1/ ROM & $\begin{array}{l}\text { ja (ersichtlich } \\
\text { aus Abbildung) }\end{array}$ & k. A. & FI-Ex, AR, LB \\
\hline Oxland et al. 1994 & HAM & 2/ ROM & ja & k. A. & FI-Ex, AR, LB \\
\hline Rousseau et al. 2006a & IAR & $4 \mathrm{IAR} / \mathrm{ROM}$ & ja & $\begin{array}{l}\text { für eine Rotation } \\
\text { von } 3^{\circ}: \text { Abwei- } \\
\text { chung der IAR= } \\
4 \mathrm{~mm} \text { und } \mathrm{Ab}- \\
\text { weichung Orien- } \\
\text { tierung }=1^{\circ}\end{array}$ & FI-Ex, LB \\
\hline Rousseau et al. 2006b & IAR & $4 \mathrm{IAR} / \mathrm{ROM}$ & ja & $\begin{array}{l}\text { für eine Rotation } \\
\text { von } 3^{\circ}: \text { Abwei- } \\
\text { chung der IAR= } \\
4 \mathrm{~mm} \text { und } \mathrm{Ab}- \\
\text { weichung Orien- } \\
\text { tierung }=1^{\circ}\end{array}$ & FI-Ex, LB \\
\hline Schulze et al. 2012 & FHA & 10/ ROM & nein & k. A. & FI-Ex \\
\hline Seligman et al.1984 & IAR & $\begin{array}{l}\text { 5-9/ ROM } \\
\text { IAR alle } 3^{\circ}\end{array}$ & nein & $\begin{array}{l}\text { für eine Rotation } \\
\text { von } 3^{\circ} \text { liegt die } \\
\text { Genauigkeit der } \\
\text { IAR Lokalisati- } \\
\text { ons-angabe bei } \\
=1 \mathrm{~mm}\end{array}$ & FI-Ex \\
\hline Sengupta et al.2011 & IAR & kontinuierlich & nein & k. A. & FI-Ex, AR, LB \\
\hline Takigawa et al. 2010 & HAM & $1 / \mathrm{ROM}$ & nein & k. A. & FI-Ex, AR, LB \\
\hline $\begin{array}{l}\text { Wachowski et al. } \\
2007\end{array}$ & $\mathrm{IHA}$ & Je $0,01^{\circ}$ & ja & $\begin{array}{l}\text { Genauigkeit für } \\
\text { Positionsvektor } \\
+/-1 \mathrm{~mm}\end{array}$ & FI-Ex, AR, LB \\
\hline $\begin{array}{l}\text { Wachowski et al. } \\
\text { 2009a }\end{array}$ & IHA & 400/ ROM & ja & $\begin{array}{l}+/-1 \mathrm{~mm} \text { Posi- } \\
\text { tionsvektor }\end{array}$ & FI-Ex, AR, LB \\
\hline $\begin{array}{l}\text { Wachowski et al. } \\
\text { 2009b }\end{array}$ & $\mathrm{IHA}$ & 400/ROM & ja & $+/-1 \mathrm{~mm}$ & AR \\
\hline $\begin{array}{l}\text { Wachowski et al. } \\
\text { 2010a }\end{array}$ & $\mathrm{IHA}$ & $\begin{array}{l}800 / \mathrm{ROM} \\
\text { In Intervallen< } \\
0,1^{\circ}\end{array}$ & ja & $+/-1 \mathrm{~mm}$ & AR \\
\hline $\begin{array}{l}\text { Wachowski et al. } \\
2010 \mathrm{~b}\end{array}$ & IHA & 400/ ROM & ja & $+/-1 \mathrm{~mm}$ & $\mathrm{AR}, \mathrm{FI}$ \\
\hline $\begin{array}{l}\text { Wachowski et al. } \\
2013\end{array}$ & $\mathrm{IHA}$ & 400/ ROM & ja & $\begin{array}{l}\text { Positions-vektor: } \\
+/-1 \mathrm{~mm}\end{array}$ & $A R$ \\
\hline
\end{tabular}




\begin{tabular}{|l|l|l|l|l|l|}
\hline $\begin{array}{l}\text { Zhu et al. } \\
\mathbf{2 0 0 7}\end{array}$ & $\begin{array}{l}\text { helical axis of } \\
\text { motion }\end{array}$ & $1 /$ ROM & ja & $\begin{array}{l}\text { Position mit } \\
\text { einer Genauig- } \\
\text { keit von } 5 \mathrm{~mm}, \\
\text { Orientierung } \\
3,0^{\circ} .\end{array}$ & FI-Ex, AR, LB \\
\hline $\begin{array}{l}\text { Zhu et al. } \\
\mathbf{2 0 1 2}\end{array}$ & HAM & $1 /$ ROM & ja & $\begin{array}{l}\text { ab Rotation von } \\
1^{\circ} \text { Fehler in der } \\
\text { Lage der HAM } \\
\text { bei bis zu 2 mm. }\end{array}$ & FI-Ex, AR, LB, \\
\hline
\end{tabular}

\subsection{Vergleich der Lokalisation errechneter Rotations-/Schraubachsen intakter zervikaler und lumbaler Segmente}

Tabelle 6 und 7 geben eine Übersicht über die zentralen Parameter der untersuchten Studien, die in den bisherigen Kapiteln bereits vorgestellt wurden. Zudem sollen auch, wenn möglich, konkrete Angaben bezüglich Lage/Wanderung und Ausrichtung der Rotations-/Schraubachse gemacht werden. Des Weiteren sollen jeweilige Änderungen des kinematischen Verhaltens der Segmente in Bezug auf Vorlastgröße und Vorlastposition (s. Kap. 3.3) angegeben werden, insofern dies in der jeweiligen Studie untersucht wurde.

\subsubsection{Zervikale Segmente}

Tab. 6 Lage und Position der Rotationsachsen (RA) bzw. Schraubachsen (SA) in zervikalen Segmenten

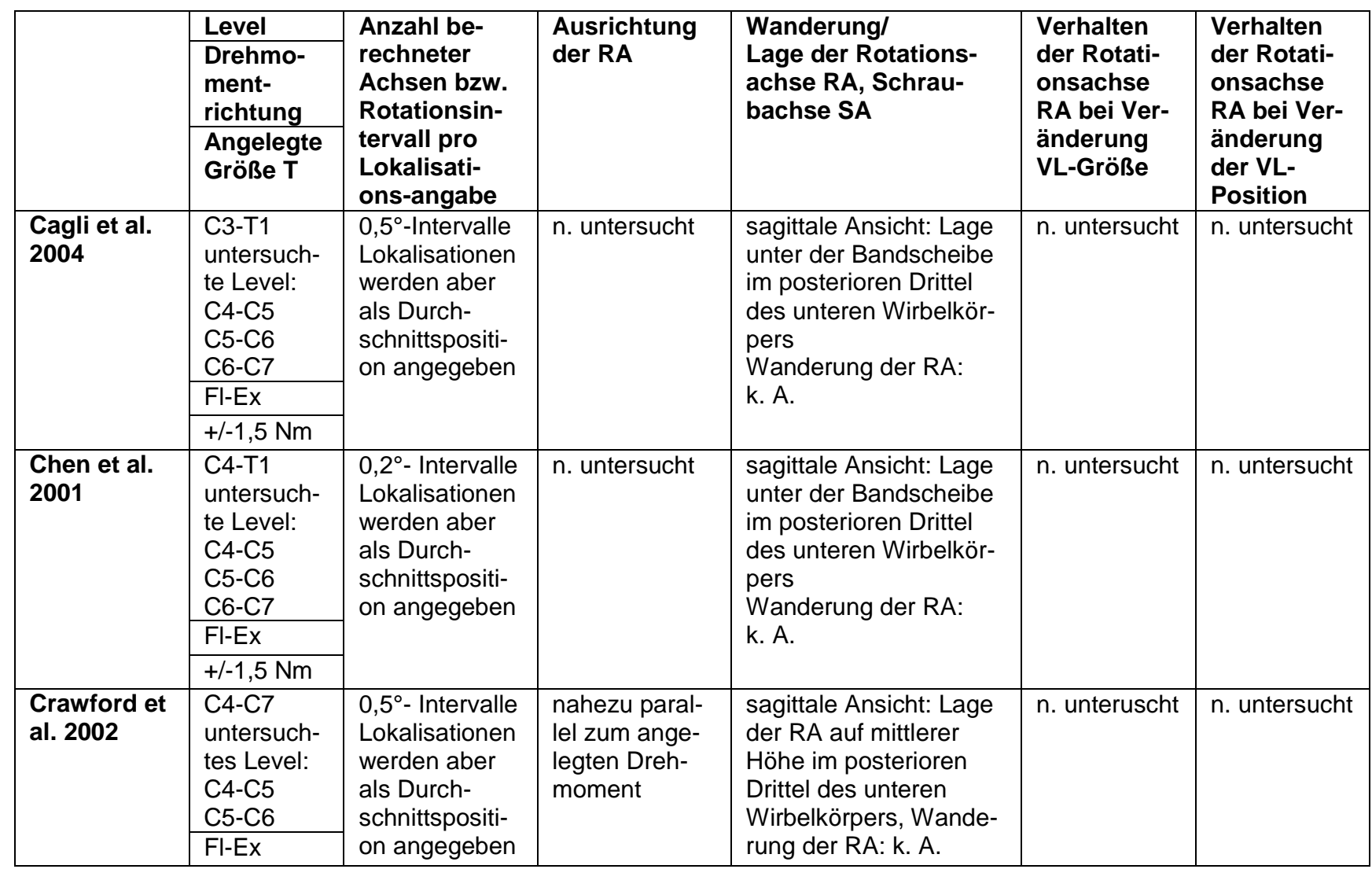




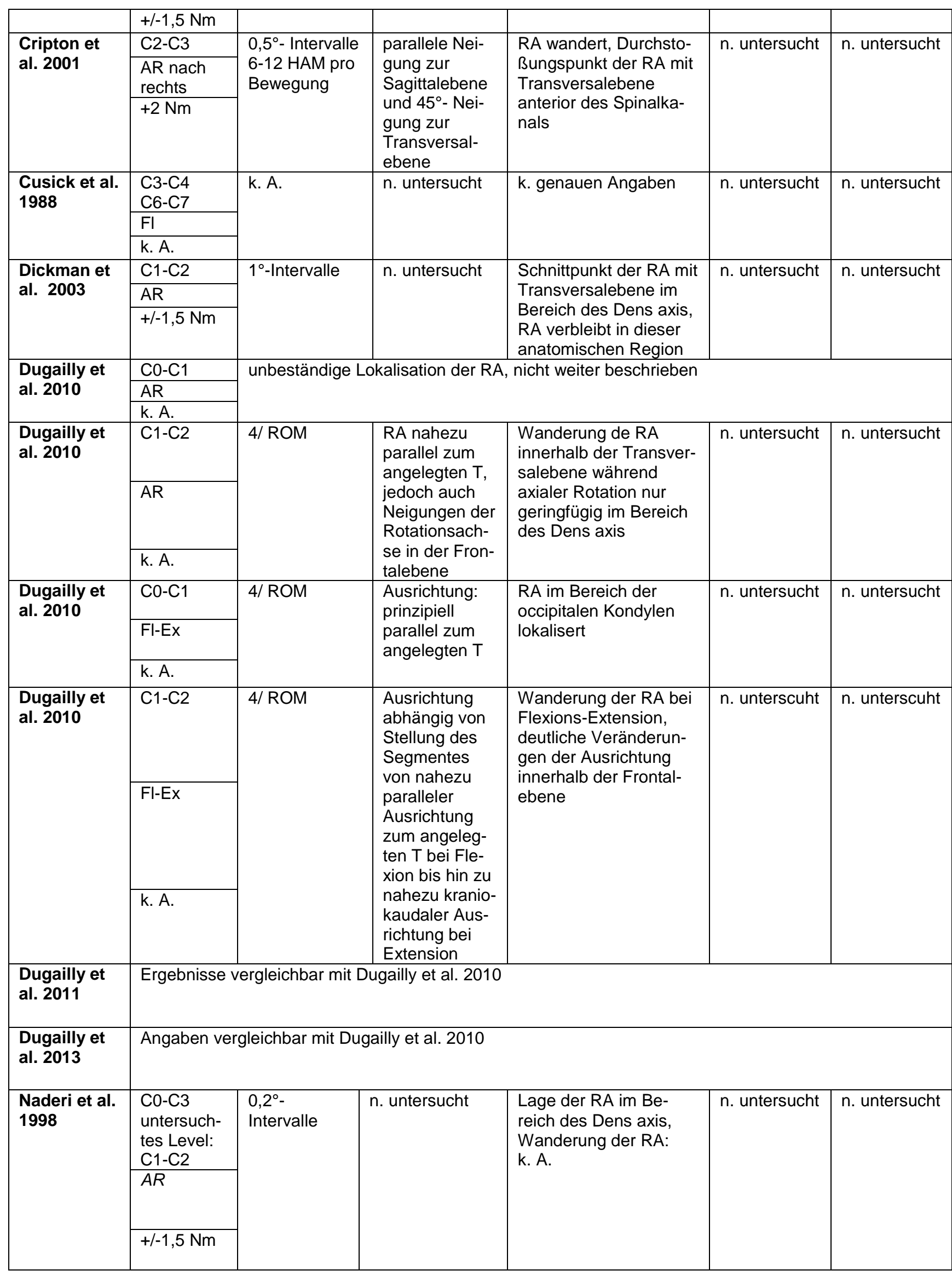




\begin{tabular}{|c|c|c|c|c|c|c|}
\hline \multirow[t]{3}{*}{$\begin{array}{l}\text { Naderi et al. } \\
1998\end{array}$} & $\begin{array}{l}\text { C0-C3 } \\
\text { untersuch- } \\
\text { tes Level: } \\
\text { C1-C2 }\end{array}$ & \multirow[t]{3}{*}{$\begin{array}{l}0,2^{\circ}-\text { Inter- } \\
\text { valle }\end{array}$} & \multirow[t]{3}{*}{ n. untersucht } & \multirow[t]{3}{*}{$\begin{array}{l}\text { Lage der RA: bei } \\
\text { sagittaler Ansicht un- } \\
\text { terhalb der Facies } \\
\text { artcularis superior } \\
\text { Wanderung der RA: } \\
\text { k. A. }\end{array}$} & \multirow[t]{3}{*}{ n. untersucht } & \multirow[t]{3}{*}{ n. untersucht } \\
\hline & FI-Ex & & & & & \\
\hline & $+/-1,5 \mathrm{Nm}$ & & & & & \\
\hline \multirow[t]{3}{*}{$\begin{array}{l}\text { Wachowski } \\
\text { et al. } 2007\end{array}$} & C3-C4 & \multirow[t]{3}{*}{$\begin{array}{l}0,01^{\circ}-\text { Inter- } \\
\text { valle }\end{array}$} & \multirow{3}{*}{$\begin{array}{l}\text { Verlauf der SA } \\
\text { von ventrokau- } \\
\text { dal nach dorsok- } \\
\text { raniel (ca. } 60^{\circ} \\
\text { zur Horizonta- } \\
\text { len) }\end{array}$} & \multirow{3}{*}{$\begin{array}{l}\text { Schnittpunkte der SA } \\
\text { mit Transversalebene } \\
\text { zeigen zum Teil abrup- } \\
\text { te Richtungsänderun- } \\
\text { gen, Wanderung der } \\
\text { RA entgegengesetzt } \\
\text { zum angelegten T, bei } \\
\text { Rotation nach rechts } \\
\text { Wanderungsstrecke } \\
\text { von } 10-20 \mathrm{~mm}\end{array}$} & \multirow[t]{3}{*}{ k. A. } & \multirow[t]{3}{*}{ k. A. } \\
\hline & AR & & & & & \\
\hline & $+/-1 \mathrm{Nm}$ & & & & & \\
\hline \multirow{3}{*}{$\begin{array}{l}\text { Wachowski } \\
\text { et al. } 2007\end{array}$} & C3-C4 & \multirow{3}{*}{$\begin{array}{l}0,01^{\circ}-\text { Inter- } \\
\text { valle }\end{array}$} & \multirow{3}{*}{$\begin{array}{l}\text { Ausrichtung } \\
\text { nahezu parallel } \\
\text { zu angelegtem T }\end{array}$} & \multirow{3}{*}{$\begin{array}{l}\text { SA unterhalb der } \\
\text { Bandscheibe, kaum } \\
\text { Wanderung der SA }\end{array}$} & \multirow[t]{3}{*}{ k. A. } & \multirow[t]{3}{*}{ k. A. } \\
\hline & FI-Ex & & & & & \\
\hline & $+/-1 \mathrm{Nm}$ & & & & & \\
\hline \multirow[t]{3}{*}{$\begin{array}{l}\text { Wachowski } \\
\text { et al. } 2007\end{array}$} & C3-C4 & \multirow[t]{3}{*}{$\begin{array}{l}0,01^{\circ}-\text { Inter- } \\
\text { valle }\end{array}$} & \multirow{3}{*}{$\begin{array}{l}\text { SA innerhalb der } \\
\text { Sagittalebene, } \\
\text { ausgepräte } \\
\text { Neigung in dor- } \\
\text { so-ventraler } \\
\text { Richtung (ca. } \\
30^{\circ} \text { zur Horizon- } \\
\text { talen) }\end{array}$} & \multirow{3}{*}{$\begin{array}{l}\text { SA mittig des Wirbel- } \\
\text { körpers C3, dann kau- } \\
\text { daler Verlauf im konve- } \\
\text { xen Bogen zur kauda- } \\
\text { len Deckplatte des } \\
\text { Wirbelkörpers C3.Bei } \\
\text { Bewegungsumkehr } \\
\text { großer Sprung der IHA } \\
\text { nach kaudal und links. } \\
\text { Dann bis mittig der } \\
\text { Bandscheibe von C3. }\end{array}$} & \multirow[t]{3}{*}{ k. A. } & \multirow[t]{3}{*}{ k. A. } \\
\hline & LB & & & & & \\
\hline & $+/-1 \mathrm{Nm}$ & & & & & \\
\hline \multirow[t]{3}{*}{$\begin{array}{l}\text { Wachowski } \\
\text { et al. 2009a }\end{array}$} & C3-C4 & \multirow[t]{3}{*}{ 400/ ROM } & \multirow{3}{*}{$\begin{array}{l}\text { SA lag innerhalb } \\
\text { der Sagittalebe- } \\
\text { ne, bei lateraler } \\
\text { Ansicht starke } \\
\text { dorso-ventrale } \\
\text { Neigung }\end{array}$} & \multirow{3}{*}{$\begin{array}{l}\text { Wanderung der SA von } \\
\text { einem Unkovertebral- } \\
\text { gelenk in einem ventral } \\
\text { konvexem Bogen zum } \\
\text { anderen Unkoverteb- } \\
\text { ralgelenk, innerhalb der } \\
\text { Rastpolkurve Kanten, } \\
\text { die abrupte Bewe- } \\
\text { gungsänderungen } \\
\text { anzeigen sollen }\end{array}$} & \multirow[t]{3}{*}{ k. A. } & \multirow[t]{3}{*}{ k. A. } \\
\hline & AR & & & & & \\
\hline & $+/-1,5 \mathrm{Nm}$ & & & & & \\
\hline \multirow{3}{*}{$\begin{array}{l}\text { Wachowski } \\
\text { et al. 2009a }\end{array}$} & C3-C4 & \multirow[t]{3}{*}{ 400/ ROM } & \multirow{3}{*}{$\begin{array}{l}\text { Ausrichtung der } \\
\text { SA nahezu } \\
\text { parallel zum } \\
\text { angelegten T }\end{array}$} & \multirow{3}{*}{$\begin{array}{l}\text { kaum Wanderung der } \\
\text { SA, SA befindet sich } \\
\text { unterhalb der Band- } \\
\text { scheibe }\end{array}$} & \multirow[t]{3}{*}{ k. A. } & k. A. \\
\hline & FI-EX & & & & & \\
\hline & $+/-1,5 \mathrm{Nm}$ & & & & & \\
\hline $\begin{array}{l}\text { Wachowski } \\
\text { et al. 2009a }\end{array}$ & C3-C4 & 400/ ROM & $\begin{array}{l}\text { nahezu parallel } \\
\text { zum angelegten }\end{array}$ & $\begin{array}{l}\text { SA mittig des Wirbel- } \\
\text { körpers C3, dann kau- }\end{array}$ & k. A. & k. A. \\
\hline & LB & & & $\begin{array}{l}\text { xen Bogen zur kauda- } \\
\text { len Deckplatte des }\end{array}$ & & \\
\hline & $+/-1,5 \mathrm{Nm}$ & & & $\begin{array}{l}\text { Wirbelkörpers C3. Bei } \\
\text { Bewegungsumkehr } \\
\text { großer Sprung der IHA } \\
\text { nach kaudal und links. } \\
\text { Dann bis mittig der } \\
\text { Bandscheibe von C3. }\end{array}$ & & \\
\hline
\end{tabular}




\begin{tabular}{|l|l|l|l|l|l|l|}
\hline $\begin{array}{l}\text { Wachowski } \\
\text { et al. 2013 }\end{array}$ & C3-C4 & 400/ ROM & $\begin{array}{l}\text { SA lag innerhalb } \\
\text { der Sagittalebe- } \\
\text { ne, bei lateraler } \\
\text { Ansicht starke } \\
\text { dorso-ventrale } \\
\text { Neigung }\end{array}$ & $\begin{array}{l}\text { Wanderung der SA } \\
\text { entgegengesetzt der } \\
\text { Bewegungsrichtung }\end{array}$ & n. untersucht & $\begin{array}{l}\text { Vorlast- } \\
\text { position hat } \\
\text { kaum Ein- } \\
\text { fluss auf } \\
\text { Ausrichtung } \\
\text { und Lage } \\
\text { der RA }\end{array}$ \\
\cline { 2 - 5 } & AR & $+/-2,24 \mathrm{Nm}$ & & & \\
\cline { 2 - 7 } & & & & & \\
\hline
\end{tabular}

\subsubsection{Zervikale Segmente axiale Rotation}

Wachowski et al. ermittelten zur Lagebestimmung der IHA bei angelegtem axialem Drehmoment für die Segmente C3/C4, Positionsvektoren der wandernden Schraubachse und bildeten die Schnittpunkte mit einer definierten Ebene ab (Rastpolkurve) (Wachowski et al. 2007, Wachowski et al. 2009a, Wachowski et al. 2013). Diese Rastpolkurve stellte einen Pfad dar, der eine Wanderung der IHA erkennen ließ. Diese, die Bewegung quantitativ beschreibende Rastpolkurve zeigte dabei abrupte Richtungsänderungen der IHA-Wanderung (Wachowski et al. 2007), die nach Angaben der Autoren Führungswechsel zwischen den Gelenken anzeigen sollten (Wachowski et al. 2007). Die IHA beschrieb dabei eine Wanderung von einem Unkovertebralgelenk in einem ventral-konvexen Bogen zum anderen Unkonvertebralgelenk (Wachowski et al. 2009a).

Bei angelegtem axialem Drehmonent fanden die Autoren für das C3/C4 Segment neben einer resultierenden axialen Rotation eine starke laterale Flexion des Segmentes im Sinne der coupled motion (s. Kap. 1.4.1) (Wachowski et al. 2007, Wachowski et al 2009a). Die IHA war dabei $30^{\circ}$ zum angreifenden Drehmoment geneigt, was einer dorsoventralen Ausrichtung der IHA entsprach (Wachowski et al. 2007, Wachowski et al. 2009a, Wachowski et al. 2013). Nach Angaben der Autoren konnte zu jedem Zeitpunkt der erfolgten Bewegung die Ausrichtung der Achse bestimmt werden (Wachowski et al. 2007, Wachowski et al. 2009a, Wachowski et al. 2013). So zeigte sich, dass die laterale Neigung der Achse kurzzeitig stark anstieg, im weiteren Verlauf der Bewegung jedoch wieder deutlich geringer wurde (Wachowski et al. 2007, Wachowski et al. 2009a).

Auch Cripton et al. gaben an, eine laterale Beugung des Segmentes im Sinne der coupled motion bei angelegter axialer Rotation nach rechts festgestellt zu haben (Cripton et al. 2001). Die HAM wurde dabei für das C2-C3 Segment errechnet und war nach Angaben der Autoren die meiste Zeit während der Bewegung nahezu pa- 
rallel zur Sagittalebene und $45^{\circ}$ in Bezug auf die Transversalebene geneigt, (Cripton et al. 2001). Die HAM verlief dabei anterior des Spinalkanals, um im weiteren Verlauf den unteren Wirbelkörper zu durchlaufen (C3) (Cripton et al. 2001).

Dickman et al. gaben an, dass sich die errechnete IAR bei angelegter Axialrotation für das Segment C1-C2 im Bereich des Dens axis, anterior des Spinalkanals, lokalisierten (Dickman et al. 1996). Vergleichbare Angaben fanden sich bei Naderi et al. (Naderi et al. 1998). Aussagen zur Orientierung der Achse machten die Autoren nicht.

Dugailly et al. errechneten für das Segment C1-C2 eine zum Dens ausgerichtete, nahezu vertikal verlaufende HA (helical axis), die ebenfalls durch den Dens von C2 verlief und somit anterior des Spinalkanals lag (Dugailly et al. 2010, Dugailly et al. 2013). Angaben zur Orientierung erfolgten in Form von Orientierungsvektoren, Abbildungen der Rotationsachse auf CT-Bildern und Angaben in Grad innnerhalb der zwei Ebenen, die nicht der Hauptbewegungsebene entsprachen (Dugailly et al. 2010, Dugailly et al. 2011). Für das Segment $\mathrm{C} 0-\mathrm{C} 1$ stellten die Autoren während der angelegten axialen Rotation eine unbeständige Lokalisation der HA fest ohne weitere Angaben zu machen. In einer weiteren Studie wurde für das gleiche Segment keine Rotationsachse berechnet, da das Bewegungsausmaß zu gering gewesen sei (Dugailly et al. 2013). Ähnliche Angaben fanden sich auch in einer weiteren Studie (Dugailly et al. 2011).

\subsubsection{Zervikale Segmente laterale Beugung}

Bei angelegter lateraler Flexion zeigte sich nach Angaben von Wachowski et al. für das C3-C4 Segment eine zusätzliche axiale Rotation des Segmentes im Sinne der coupled motion (Wachowski et al. 2007, Wachowski et al. 2009a). Wie aus den Richtungsvektoren ersichtlich wurde, lag die Schraubachse dabei nahezu parallel zur Sagittalebene. In Bezug auf das angelegte Drehmoment zeigte sich annähernd eine $30^{\circ}$ Neigung der Achsschar (Wachowski et al. 2007, Wachowski et al. 2009a).

Die Rastpolkurve in der Frontalebene zeigte dabei eine Wanderung, die bei Linksrotation mittig des Wirbelkörpers C3 begann um nach einem kaudalen Verlauf und einem konvexen Bogen zur kaudalen Deckplatte des Wirbelkörpers C3 zu verlaufen. Bei Bewegungsumkehr zeigte sich ein großer Sprung der IHA nach kaudal und links. Die Rastpolkurve verlief dann wieder in Richtung der Mitte des Wirbelkörpers C3 bis 
etwa mittig der Bandscheibe von C3 (Wachowski et al. 2007, Wachowski et al. 2009a).

Es fanden sich keine weiteren Studien, die eine Rotations-/Schraubachse für zervikale Segmente und angelegtes Drehmoment für laterale Beugungen berechneten. Naderi et al. gaben hierzu an, dass die interindividuellen Unterschiede und die Bewegungen, die außerhalb der Frontalebene, in der der Hauptteil der Bewegung stattfinden sollte, zu groß seien, um eine Rotationsachse für laterale Bewegungen berechnen zu können (Naderi et al. 1998).

\subsubsection{Zervikale Segmente Flexion-Extension}

Chen et al. vereinheitlichten die Position der IAR und gaben an, dass sich die IAR für die Level C4-C5, C5-C6 und C6-C7 bei sagittaler Ansicht posterior des Mittelpunktes im kranialen Bereich des jeweils unteren Wirbelkörpers befanden (Chen et al. 2001). Vergleichbare Angaben fanden sich bei Cagli et al. (2004) für die Segmente C5-C6.

Für die Segmente C1-C2 zeigte sich bei Naderi et al., dass sich die IAR direkt kaudal der Facies articularis superior befunden habe (Naderi et al. 1998).

Eine dreidimensionale Betrachtung stellten hingegen Dugailly et al. an. Die Autoren gaben an, dass sich die errechnete helical axis (HA) für das Segment C0-C1 im Bereich der occipitalen Kondylen befand (Dugailly et al. 2010, Dugailly et al. 2011). Die Achse wies nur eine geringe Neigung in Bezug zum angelegten Drehmoment auf (Dugailly et al. 2010). Ähnliche Angaben fanden sich in weiteren Studien (Dugailly et al. 2011, Dugailly et a. 2013). Auch Wachowski et al. gaben an, dass bei FlexionExtension des C3-C4 Segmentes sich eine zum angelegten Drehmoment annähernd parallele Achsschar der IHA zeigte. Die IHA lokalisierte sich dabei unterhalb der Bandscheibe (Wachowski et al. 2007, Wachowski et al. 2009a). Eine ähnliche Orientierung zeigte sich auch bei Crawford et al. Die axis of rotation lag dabei für die Segmente C4-5 bzw. C5-C6 auf mittlerer Höhe im posterioren Drittel des unteren Wirbelkörpers (Crawford et al. 2002).

Ein stark zu den genannten Angaben abweichendes Verfahren fand sich bei Cusick et al. Hier wurde ein Wirbelkörper in drei Bereiche eingeteilt (Proc. spinosus, Facettengelenke, Wirbelkörper). Für jeden der Bereiche wurde der prozentuale Unterschied der Bewegung für Segmente nach bilateraler Gelenkentfernung und bilateraler Gelenkfixierung im Vergleich zum intakten Segment dargestellt. Die Autoren 
schlussfolgerten aus einer verringerten Bewegung, dass sich die IAR in diesem Bereich befunden haben müsse, da in den Bereichen nahe der Rotationsachse weniger Bewegung erwartet wurde. Die Position der IAR wurde demnach nur in tendenziellen Verlagerungen der Bewegung nach anterior bzw. posterior zwischen den verschiedenen Testbedingungen beschrieben. So zeigte die Gelenkentfernung eine tendenzielle Anteriorverlagerung der IAR, wohingegen die Gelenkfixierung eine posteriore Verlagerung der IAR bewirkt hätte (Cusick et al. 1988).

\subsubsection{Lumbale Segmente}

Tab. 7 Lage und Position der Rotationsachsen (RA) bzw. Schraubachsen (SA) in lumbalen Segmenten

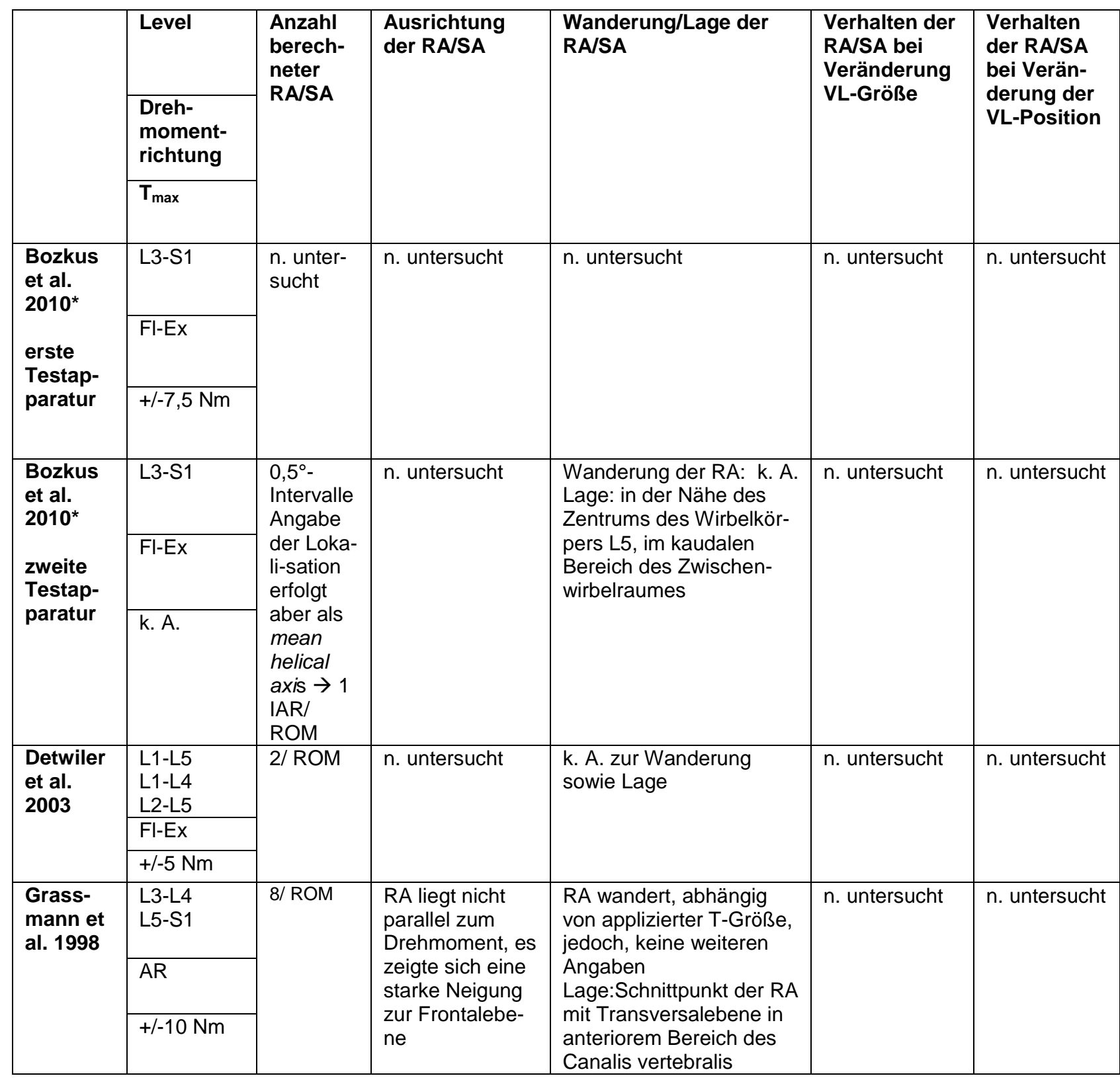




\begin{tabular}{|c|c|c|c|c|c|c|}
\hline $\begin{array}{l}\text { Haberl et } \\
\text { al. } 2004\end{array}$ & $+/-12 \mathrm{Nm}$ & $\begin{array}{l}5 / 1 / 2 \\
\text { ROM }\end{array}$ & $\begin{array}{l}\text { nahezu parallel } \\
\text { zu angelegtemT }\end{array}$ & $\begin{array}{l}\text { Schnittpunkt der HAM mit } \\
\text { der Transversalebene } \\
\text { (bezogen auf die obere } \\
\text { Endplatte des unteren } \\
\text { Wirbelkörpers) links } \\
\text { bzw.-rechts anterior- } \\
\text { lateral des Canalis ver- } \\
\text { tebralis, Wanderung der } \\
\text { RA entgegengesetzt von } \\
\text { T }\end{array}$ & $\begin{array}{l}\text { VL-Applikation } \\
\text { führt je nach } \\
\text { Position der } \\
\text { VL zu erhebli- } \\
\text { chen Verände- } \\
\text { rungen der } \\
\text { Position der } \\
\text { HAM (s. Text) }\end{array}$ & $\begin{array}{l}\text { zunächst } \\
\text { Anterior- } \\
\text { verlagerung } \\
\text { der HAM bei } \\
\text { zentraler VL, } \\
\text { jedoch } \\
\text { posterior } \\
\text { Verlagerung } \\
\text { der HAM bei } \\
\text { Verlagerung } \\
\text { der VL nach } \\
\text { anterior oder } \\
\text { posterior }\end{array}$ \\
\hline \multirow[t]{2}{*}{$\begin{array}{l}\text { Kettler } \\
\text { et al. } \\
2004\end{array}$} & L4-L5 & \multirow[t]{2}{*}{$\begin{array}{l}0,1^{\circ}- \\
\text { Intervalle }\end{array}$} & \multirow{2}{*}{$\begin{array}{l}\text { große interindi- } \\
\text { viduelle Unter- } \\
\text { schiede zum } \\
\text { Teil parallele } \\
\text { Ausrichtung der } \\
\text { RA zu T, teilwei- } \\
\text { se mit anteriorer } \\
\text { Neigung }\end{array}$} & \multirow[t]{2}{*}{$\begin{array}{l}\text { RA nahe der Facettenge- } \\
\text { lenke, k. A. zur Wande- } \\
\text { rung }\end{array}$} & \multirow[t]{2}{*}{ n. untersucht } & \multirow[t]{2}{*}{ n. unteruscht } \\
\hline & k. A. & & & & & \\
\hline \multirow[t]{2}{*}{$\begin{array}{l}\text { Kettler } \\
\text { et al. } \\
2004\end{array}$} & L4-L5 & \multirow[t]{2}{*}{$\begin{array}{l}0,1^{\circ}- \\
\text { Intervalle }\end{array}$} & \multirow[t]{2}{*}{$\begin{array}{l}\text { nahezu parallel } \\
\text { zu T, in Bezug } \\
\text { auf die Ausrich- } \\
\text { tung geringe } \\
\text { interindividuelle } \\
\text { Unterschiede }\end{array}$} & \multirow[t]{2}{*}{$\begin{array}{l}\text { Verlauf entweder durch } \\
\text { die Bandscheibe oder } \\
\text { den unteren Wirbelkörper } \\
\text { verliefen, k. A. zur Wan- } \\
\text { derung }\end{array}$} & \multirow[t]{2}{*}{ n. untersucht } & \multirow[t]{2}{*}{ n. untersucht } \\
\hline & k. A. & & & & & \\
\hline $\begin{array}{l}\text { Kettler } \\
\text { et al. } \\
2004\end{array}$ & L4-L5 & $\begin{array}{l}0,1^{\circ}- \\
\text { Intervalle }\end{array}$ & $\begin{array}{l}\text { große interindi- } \\
\text { viduelle Unter- } \\
\text { schiede inner- } \\
\text { halb der Hori- } \\
\text { zontalebene } \\
\text { zum Teil fächer- } \\
\text { förmige Streu- } \\
\text { ung, zum Teil } \\
\text { parallele Aus- } \\
\text { richtung in ante- } \\
\text { rior-posteriorer } \\
\text { Richtung }\end{array}$ & $\begin{array}{l}\text { zwei mögliche Verlaufs- } \\
\text { formen der RA: zum } \\
\text { einen durchläuft die RA } \\
\text { in der Horizontalebene } \\
\text { den Wirbelkörper mittig } \\
\text { zum anderen zeigte sich } \\
\text { eine starke Streuung der } \\
\text { Achsschar innerhalb der } \\
\text { Horizontalebene mit } \\
\text { einem zentralen Kreu- } \\
\text { zungspunkt der Achsen } \\
\text { im anterioren Drittel der } \\
\text { Bandscheibe, } \\
\text { k. A. zur Wanderung }\end{array}$ & n. untersucht & n. untersucht \\
\hline \multirow{2}{*}{$\begin{array}{l}\text { Mansour } \\
\text { et al. } \\
2004\end{array}$} & L3-L4 & \multirow{2}{*}{$\begin{array}{l}0,02^{\circ}- \\
\text { Intervalle }\end{array}$} & \multirow{2}{*}{$\begin{array}{l}\text { nahezu parallel } \\
\text { zum angelegten } \\
\mathrm{T}\end{array}$} & \multirow{2}{*}{$\begin{array}{l}\text { Wanderung der RA von } \\
\text { einem Facettengelenk in } \\
\text { einem ventralen Bogen } \\
\text { zum anderen Facetten- } \\
\text { gelenk, Bewegungsstre- } \\
\text { cke } 60 \mathrm{~mm}\end{array}$} & \multirow[t]{2}{*}{ n. untersucht } & \multirow[t]{2}{*}{ n. untersucht } \\
\hline & $+/-30 \mathrm{Nm}$ & & & & & \\
\hline $\begin{array}{l}\text { McNally } \\
\text { et al. } \\
2012\end{array}$ & $\begin{array}{l}\text { L3-L4 und } \\
\text { L4-L5 }\end{array}$ & k. A. & k. A. & $\begin{array}{l}\text { RA in neutraler Stellung } \\
\text { in Nähe des Zentrums } \\
\text { der Bandscheibe im } \\
\text { kaudalen Bereich des } \\
\text { Zwischenwirbelraumes. } \\
\text { Bei Flexion Wanderung } \\
\text { nach dorsal }\end{array}$ & k. A. & $\begin{array}{l}\text { VL- } \\
\text { Verlagerung } \\
\text { nach anteri- } \\
\text { or: dorsal } \\
\text { Verlagerung } \\
\text { der RA nach } \\
\text { dorsal }\end{array}$ \\
\hline $\begin{array}{l}\text { Nägerl et } \\
\text { al. } 2009\end{array}$ & $\begin{array}{l}\text { L3-L4 und } \\
\text { L4-L5 } \\
\text { FI-Ex }\end{array}$ & $\begin{array}{l}\text { 400/ } \\
\text { ROM }\end{array}$ & $\begin{array}{l}\text { nahezu parallel } \\
\text { zum angelegtem } \\
\mathrm{T} \text { unabhängig } \\
\text { von der VL }\end{array}$ & $\begin{array}{l}\text { RA in neutraler Stellung } \\
\text { unter der Bandscheibe } \\
\text { des Segmentes, wenig } \\
\text { Wanderung bei Flexions- } \\
\text { bewegungen, ausgepräg- } \\
\text { te Dorsalwanderung der } \\
\text { Rastpolkurve in der } \\
\text { Sagittalebene bei Exten- } \\
\text { sion }\end{array}$ & n. untersucht & $\begin{array}{l}\text { Dorsalverla- } \\
\text { gerung der } \\
\text { VL: tenden- } \\
\text { ziell Dorsal- } \\
\text { wanderung } \\
\text { der IHA }\end{array}$ \\
\hline
\end{tabular}




\begin{tabular}{|c|c|c|c|c|c|c|}
\hline $\begin{array}{l}\text { Niosi et } \\
\text { al. } 2006\end{array}$ & $\begin{array}{l}\text { L2-L5 } \\
\text { untersuch- } \\
\text { tes Level } \\
\text { L3-L4 } \\
\text { AR } \\
\end{array}$ & 1/ ROM & $\begin{array}{l}\text { ohne VL: RA } \\
\text { nahezu parallel } \\
\text { zum angelegtem } \\
\mathrm{T}\end{array}$ & $\begin{array}{l}\text { Wanderung der RA: k. A. } \\
\text { Lage: Schnittpunkt der } \\
\text { RA mit Transversalebe- } \\
\text { ne an der anterioren } \\
\text { Grenze des Canalis } \\
\text { vertebralis }\end{array}$ & $\begin{array}{l}\text { mit VL: } \\
\text { dorsoventrale } \\
\text { Neigung ent- } \\
\text { gegengesetzt } \\
\text { der Uhrzeiger- } \\
\text { richtung (bei } \\
\text { linkslateraler } \\
\text { Betrachtung) } \\
\text { stärker aus- } \\
\text { geprägt }\end{array}$ & n. untersucht \\
\hline & $+/-7,5 \mathrm{Nm}$ & & & & & \\
\hline $\begin{array}{l}\text { Niosi et } \\
\text { al. } 2006\end{array}$ & $\begin{array}{l}\text { L2-L5 } \\
\text { untersuch- } \\
\text { tes Level } \\
\text { L3-L4 } \\
\text { FI-EX } \\
+/-/, 5 \mathrm{Nm}\end{array}$ & 1/ ROM & $\begin{array}{l}\text { ohne VL: nahe- } \\
\text { zu parallel zum } \\
\text { angelegten T }\end{array}$ & $\begin{array}{l}\text { Wanderung der RA: k. A. } \\
\text { Lage in sagittaler An- } \\
\text { sicht: im Zwischenwirbel- } \\
\text { bereich, etwa mittig der } \\
\text { Bandscheibe }\end{array}$ & $\begin{array}{l}\text { mit VL signifi- } \\
\text { kante Rotation } \\
\text { der HAM in } \\
\text { Uhrzeigerrich- } \\
\text { tung* in der } \\
\text { Transversal- } \\
\text { ebene }\end{array}$ & n. untersucht \\
\hline $\begin{array}{l}\text { Niosi et } \\
\text { al. } 2006\end{array}$ & $\begin{array}{l}\text { L2-L5 } \\
\text { untersuch- } \\
\text { tes Level } \\
\text { L3-L4 } \\
\text { LB } \\
+/-7,5 \mathrm{Nm}\end{array}$ & 1/ ROM & $\begin{array}{l}\text { ohne VL: nahe- } \\
\text { zu parallel zum } \\
\text { angelegten T }\end{array}$ & $\begin{array}{l}\text { Wanderung der RA: k. A. } \\
\text { Lage von frontaler An- } \\
\text { sicht: im kaudalen Be- } \\
\text { reich des oberen Wirbel- } \\
\text { körpers (L3) }\end{array}$ & $\begin{array}{l}\text { VL hat kaum } \\
\text { Einfluss auf } \\
\text { Orientierung } \\
\text { der HAM }\end{array}$ & n. untersucht \\
\hline $\begin{array}{l}\text { Sen- } \\
\text { gupta et } \\
\text { al. } 2011\end{array}$ & $\begin{array}{l}\text { L3-L5 } \\
\text { AR }\end{array}$ & $\begin{array}{l}\text { kontinu- } \\
\text { ierlich }\end{array}$ & n. untersucht & $\begin{array}{l}\text { entgegengesetzt der } \\
\text { angelegten Drehmoment- } \\
\text { richtung verlaufend, } \\
\text { innerhalb der Transver- } \\
\text { saleebene: initiale Positi- } \\
\text { on im anterioren Bereich } \\
\text { des Wirbelkörpers, dann } \\
\text { Wanderung der RA in } \\
\text { Richtung linkem/rechtem } \\
\text { Facettengelenk, Wande- } \\
\text { rungsstrecke etwa } \\
60 \text { mm }\end{array}$ & n. untersucht & n. untersucht \\
\hline $\begin{array}{l}\text { Sen- } \\
\text { gupta et } \\
\text { al. } 2011\end{array}$ & $\begin{array}{l}\text { L3-L5 } \\
\text { FI-Ex } \\
\text { k. A. }\end{array}$ & $\begin{array}{l}\text { kontinu- } \\
\text { ierlich }\end{array}$ & n. untersucht & $\begin{array}{l}\text { Bewegung hauptsächlich } \\
\text { in Sagittalebene mit } \\
\text { Wansderungsstrecken } \\
\text { von bis zu } 26.5 \mathrm{~mm}\end{array}$ & n. untersucht & n. untersucht \\
\hline $\begin{array}{l}\text { Sen- } \\
\text { gupta et } \\
\text { al. } 2011\end{array}$ & $\begin{array}{l}\text { L3-L5 } \\
\text { LB } \\
\text { k. A. }\end{array}$ & $\begin{array}{l}\text { kontinu- } \\
\text { ierlich }\end{array}$ & n. untersucht & $\begin{array}{l}\text { bei posteriorer Ansicht: } \\
\text { RA zentral im Zwischen- } \\
\text { wirbelbereich, Bewe- } \\
\text { gungsstrecke in Frontal- } \\
\text { ebene von bis zu } \\
15.35 \mathrm{~mm}\end{array}$ & n. untersucht & n. untersucht \\
\hline $\begin{array}{l}\text { Takiga- } \\
\text { wa et al. } \\
2010\end{array}$ & $+/-7.5 \mathrm{Nm}$ & 1/ ROM & $\begin{array}{l}\text { nahezu parallel } \\
\text { zu T }\end{array}$ & $\begin{array}{l}\text { Schnittpunkt der HAM mit } \\
\text { der Transversalebene für } \\
\text { L4-L5 Segmente im ante- } \\
\text { rioren Bereich des Cana- } \\
\text { lis vertebralis, keine } \\
\text { Angaben zur Wanderung }\end{array}$ & $\begin{array}{l}\text { Applikation } \\
\text { einer VL: leich- } \\
\text { te Dorsalver- } \\
\text { lagerung der } \\
\text { RA, nahezu } \\
\text { keine Verän- } \\
\text { derung der } \\
\text { Orientierung }\end{array}$ & n. untersucht \\
\hline
\end{tabular}




\begin{tabular}{|c|c|c|c|c|c|c|}
\hline \multirow{3}{*}{$\begin{array}{l}\text { Takiga- } \\
\text { wa et al. } \\
2010\end{array}$} & L4-L5 & \multirow[t]{3}{*}{ 1/ ROM } & \multirow[t]{3}{*}{$\begin{array}{l}\text { nahezu parallel } \\
\text { zu T }\end{array}$} & \multirow{3}{*}{$\begin{array}{l}\text { Lage der RA zentral im } \\
\text { Zwischenwirbelbereich, } \\
\text { Wanderung der RA: } \mathrm{k} \text {. A. }\end{array}$} & \multirow{3}{*}{$\begin{array}{l}\text { Applikation } \\
\text { einer VL: Kau- } \\
\text { dalverlagerung } \\
\text { der HAM, } \\
\text { nahezu keine } \\
\text { Veränderung } \\
\text { der Orientie- } \\
\text { rung }\end{array}$} & \multirow[t]{3}{*}{ n. untersucht } \\
\hline & Fl-Ex & & & & & \\
\hline & $+/-7.5 \mathrm{Nm}$ & & & & & \\
\hline \multirow{4}{*}{$\begin{array}{l}\text { Takiga- } \\
\text { wa et al. } \\
2010\end{array}$} & L4-L5 & \multirow[t]{4}{*}{ 1/ ROM } & \multirow{4}{*}{$\begin{array}{l}\text { nahezu parallel } \\
\text { zu T }\end{array}$} & \multirow{4}{*}{$\begin{array}{l}\text { RA liegt in der Frontal- } \\
\text { ebene im kaudalen Be- } \\
\text { reich, zentral des oberen } \\
\text { Wirbelkörpers, } \\
\text { Wanderung der RA: k. A. }\end{array}$} & \multirow{4}{*}{$\begin{array}{l}\text { Applikation } \\
\text { einer VL: Kau- } \\
\text { dalverlagerung } \\
\text { der HAM na- } \\
\text { hezu keine } \\
\text { Veränderung } \\
\text { der Orientie- } \\
\text { rung }\end{array}$} & \multirow[t]{4}{*}{ n. untersucht } \\
\hline & & & & & & \\
\hline & LB & & & & & \\
\hline & $+/-7.5 \mathrm{Nm}$ & & & & & \\
\hline \multirow[t]{3}{*}{$\begin{array}{l}\text { Wach- } \\
\text { owski et } \\
\text { al. 2009a }\end{array}$} & L3-L4 & \multirow[t]{3}{*}{$\begin{array}{l}400 / \\
\text { ROM }\end{array}$} & \multirow[t]{3}{*}{$\begin{array}{l}\text { nahezu parallel } \\
\text { zu T unabhängig } \\
\text { von VL und T- } \\
\text { Größe }\end{array}$} & \multirow{3}{*}{$\begin{array}{l}\text { Wanderung von einem } \\
\text { Facettengelenk zum } \\
\text { anderen in einem ventra- } \\
\text { len Bogen, Verlauf ent- } \\
\text { gegengesetzt zum ange- } \\
\text { legtem T (Wanderungs- } \\
\text { strecke der RA bis zu } \\
60 \mathrm{~mm} \text { ) }\end{array}$} & \multirow[t]{3}{*}{ k. A. } & \multirow{3}{*}{$\begin{array}{l}\text { anteriore VL: } \\
\text { anteriore } \\
\text { Verlagerung } \\
\text { der RA, } \\
\text { dorsale VL: } \\
\text { Dorsalverla- } \\
\text { gerung der } \\
\text { RA abhängig } \\
\text { von VL: } \\
\text { ventraler } \\
\text { bzw. dorsa- } \\
\text { ler Bogen }\end{array}$} \\
\hline & $\overline{A R}$ & & & & & \\
\hline & $+/-30 \mathrm{Nm}$ & & & & & \\
\hline \multirow{3}{*}{$\begin{array}{l}\text { Wach- } \\
\text { owski et } \\
\text { al.2009a }\end{array}$} & L3-L4 & \multirow{3}{*}{$\begin{array}{l}400 / \\
\text { ROM }\end{array}$} & \multirow{3}{*}{$\begin{array}{l}\text { nahezu parallel } \\
\text { zu T }\end{array}$} & \multirow{3}{*}{$\begin{array}{l}\text { RA initial im dorsalen } \\
\text { Bereich des Spinalka- } \\
\text { nals, Flexion: Wanderung } \\
\text { der RA (Wanderungs- } \\
\text { strecke } 20 \mathrm{~mm} \text { ) in Rich- } \\
\text { tung Zentrum der Band- } \\
\text { scheibe } \\
\text { Extension: Dorsalwande- } \\
\text { rung der RA zu den Fa- } \\
\text { cettengelenken }\end{array}$} & \multirow[t]{3}{*}{ k. A. } & \multirow[t]{3}{*}{ k. A. } \\
\hline & FI-Ex & & & & & \\
\hline & $+/-6 \mathrm{Nm}$ & & & & & \\
\hline \multirow[t]{3}{*}{$\begin{array}{l}\text { Wach- } \\
\text { owski } \\
\text { et al. } \\
2009 a\end{array}$} & L3-L4 & \multirow[t]{3}{*}{$\begin{array}{l}400 / \\
\text { ROM }\end{array}$} & \multirow[t]{3}{*}{$\begin{array}{l}\text { nahezu parallel } \\
\text { zu T }\end{array}$} & \multirow{3}{*}{$\begin{array}{l}\text { Lage der RA abhängig } \\
\text { von Vorlastposition, bei } \\
\text { Dorsalverlagerung der } \\
\text { VL: Wanderung der RA } \\
\text { bei frontaler Ansicht von } \\
\text { einem Facettengelenk } \\
\text { zum anderen (Wande- } \\
\text { rungsstrecke bis zu } \\
40 \mathrm{~mm} \text { ) }\end{array}$} & \multirow[t]{3}{*}{ k. A. } & \multirow{3}{*}{$\begin{array}{l}\text { Dorsalverla- } \\
\text { gerung der } \\
\text { VL: RA wei- } \\
\text { ter kranial } \\
\text { als bei Ver- } \\
\text { lagerung der } \\
\text { VL nach } \\
\text { anterior, } \\
\text { Verlagerung } \\
\text { der VL nach } \\
\text { anterior: } \\
\text { zunächst } \\
\text { parallel dann } \\
\text { Drehung } \\
\text { innerhalb der } \\
\text { Sagittalebe- } \\
\text { ne }\end{array}$} \\
\hline & LB & & & & & \\
\hline & $+/-20 \mathrm{Nm}$ & & & & & \\
\hline \multirow{3}{*}{$\begin{array}{l}\text { Wach- } \\
\text { owski } \\
\text { et al. } \\
2010 a\end{array}$} & $\begin{array}{l}\text { L3-L4 } \\
\text { L4-L5 }\end{array}$ & \multirow[t]{3}{*}{$\begin{array}{l}400 / \\
\text { ROM }\end{array}$} & \multirow[t]{3}{*}{$\begin{array}{l}\text { nahezu parallel } \\
\text { zu T }\end{array}$} & \multirow{3}{*}{$\begin{array}{l}\text { Wanderung der RA von } \\
\text { einem Facettelngelenk } \\
\text { zum andere entgegenge- } \\
\text { setzt der Bewegungsrich- } \\
\text { tung }\end{array}$} & \multirow[t]{3}{*}{ k. A. } & \multirow{3}{*}{$\begin{array}{l}\text { Dorsalverla- } \\
\text { gerung der } \\
\text { VL: Dorsal- } \\
\text { verlagerung } \\
\text { der RA, } \\
\text { Anteriorver- } \\
\text { lagerung der } \\
\text { VL: Anterior- } \\
\text { verlagerung } \\
\text { der RA }\end{array}$} \\
\hline & AR & & & & & \\
\hline & $+/-32,4 \mathrm{Nm}$ & & & & & \\
\hline
\end{tabular}




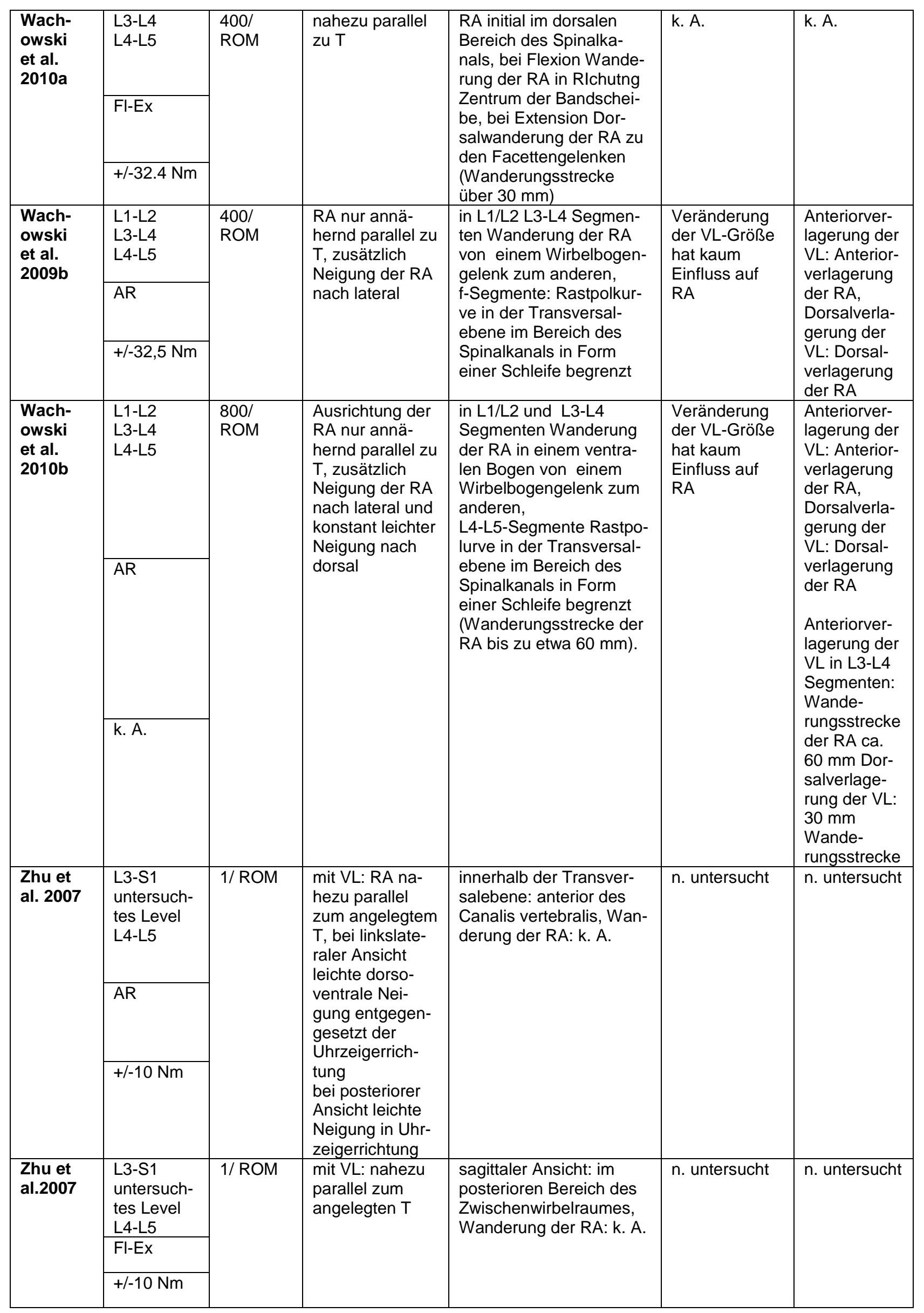




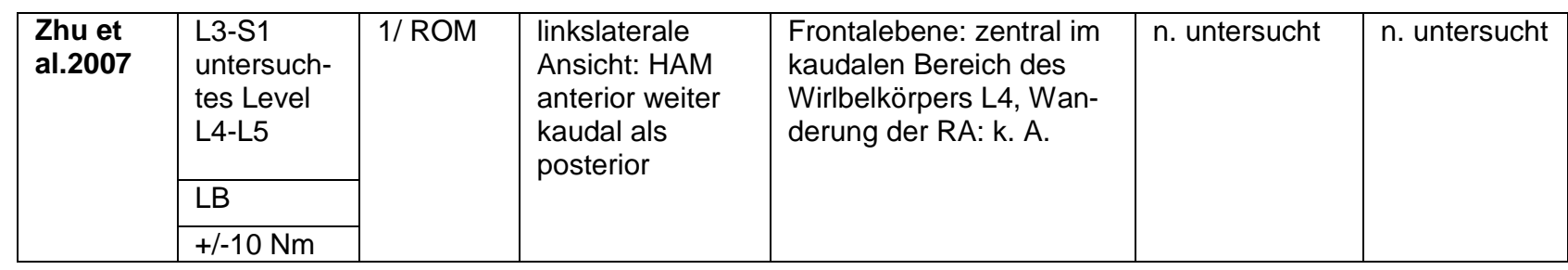

\subsubsection{Lumbale Segmente axiale Rotation}

Grassmann et al. gaben an, dass sich die errechnete helical axis (HA) bei axialer Rotation in den meisten Fällen in einem anterioren Bereich des Canalis vertebralis befand. Weiterhin gaben die Autoren an, dass die HA eine starke Neigung zur Frontalebene hatte. Dies führten die Autoren auf laterale Rotation des Segmentes im Sinne der coupled motion (s. Kap. 1.4.1) zurück. Die genannten Feststellungen wurden für L3-L4 und L5-S1 Segmente vereinheitlicht. Darstellungen des Verlaufs der HAM auf Abbildungen oder Angaben der Orientierung in Richtungsvektoren bzw. Gradzahlen der Neigung in einer Ebene erfolgten nicht (Grassmann et al. 1998). Auch Sengupta et al. machten keine Aussagen zur Orientierung der Rotationsachse. Die Autoren stellten fest, dass der Schnittpunkt der errechneten IAR mit der Transversalebene für L4-L5 Segmente bei axialer Rotation, einen halbkreisförmigen Bogen darstellte, der in entgegengesetzter Richtung des angelegten Drehmoments vom anterioren Drittel des Wirbelkörpers in neutraler Stellung in Richtung linkem bzw. rechtem Wirbelbogengelenk verlief und eine Länge von etwa $60 \mathrm{~mm}$ aufwies (Sengupta et al. 2011). Ähnliche Angaben fanden sich bei Mansour et al (2004). Hier lag für L3-L4 Segmente, bei angelegtem axialen Drehmoment, die Schraubachse im anterioren Bereich des Wirbelkörpers. Die Rastpolkurve verlief in einem ventralen Bogen um den Canalis vertebralis, wie auch von Sengupta et al. (2011) beschrieben. Dabei legte die IHA von einem Wirbelbogengelenk zum anderen eine Strecke von $60 \mathrm{~mm} \mathrm{zu}$ rück. Die IHA war dabei fast ausschließlich annähernd parallel zum angelegten Drehmoment (Mansour et al. 2004). Ähnliche Angaben fanden sich auch bei Wachowski et al.: Die Autoren gaben an, dass in L3-L4 Segmenten die IHA von einem Wirbelbogengelenk zum anderen wanderte, abhängig von der Position der Vorlast jedoch in einem ventralen (bei Verlagerung der VL nach anterior) bzw. dorsalen Bogen (bei Verlagerung der VL nach posterior) (Wachowski et al. 2007, Wachowski et al. 2009a, Wachowski et al. 2009b, Wachowski et al. 2010). Die größte Strecke lege die IHA dabei innerhalb eines Intervalls von $+/-1^{\circ}$ zurück (Wachowski et al. 2009b). 
Andere Lokalisationen der IHA fanden sich für L4-L5-Segmente. Hier war die Rastpolkurve der IHA im Bereich des Spinalkanals in Form einer Schleife begrenzt. Auch hier zeigte die Position der Vorlast einen Einfluss auf die Wanderung der Achse. Dorsal-Position der VL begrenzte den Verlauf der Rastpolkurve auf einen noch kleineren Bereich, Ventral-Position der Vorlast führte hingegen zu einem Verlauf der Rastpolkurve von einem Bereich nahe des einen Wirbelbogengelenks zum anderen (Wachowski et al. 2009b, Wachowski et al. 2010) (Abb. 15).

Auch Haberl et al. (2004) gaben an, dass die Rottionsachse entgegengesetzt der Drehbewegung wanderte und erkannten ebenfalls einen Effekt der Vorlastverschiebung auf die Lage der HAM. Jedoch zeigte sich hier im Unterschied zu den zuvor genannten Studien, dass nach Anterior- oder Posteriorverschiebung der Vorlast bei angelegtem axialen Drehmoment die HAM in beiden Fällen eine Posteriorverlagerung vollzog. Abb. 16 und 17 zeigen die Lage der HAM innerhalb de Transversalebene in Abhängigkeit der Vorlast. Die HAM zeigte dabei eine größtenteils vertikale Ausrichtung (Haberl et al. 2004).

Eine ähnliche Lokalisation der HAM wie zuvor von Haberl et al. (2004) beschrieben, (s. Abb. 16, 17) fand sich auch bei Niosi et al. (2006) für L3-L4 bzw. bei Zhu et al. (2007) und Takigawa et al. (2010) für L4-L5 Segmente. Hier lag die HAM an der anterioren Grenze des Canalis vertebraliis. Bei Niosi et al. (2006) und Takigawa et al. (2010) zeigte sich innerhalb der Frontalebene ein nahezu vertikaler Verlauf, von sagittal hingegen eine leichte dorso-ventrale Neigung der HAM (Niosi et al. 2006, Zhu et al. 2007), die nach Niosi et al. (2006) ohne Applikation einer Vorlast weniger stark ausgeprägt war. Bei Takigawa et al. zeigte sich für die getesteten L4-L5 Segmente ein nahezu senkrechter Verlauf der HAM (Takigawa et al. 2010). Bei Applikation einer Vorlast von $400 \mathrm{~N}$ wanderte der Schnittpunkt der HAM nach kranial und anterior (s. Abb. 13). Auf die Orientierung hatte die Vorlast wenig Einfluss. Bei Takigawa et al. zeigte sich ein nahezu vertikaler Verlauf der HAM (Takigawa et al. 2010).

Kettler et al. gaben an, dass sich die FHA für das untersuchte Segment L4-L5 in der Nähe der Facettengelenke im posterioren Bereich der Bandscheibe befanden (s. Abb. 14). Dabei stellten die Autoren große interindividuelle Unterschiede fest. So 
fanden sich nach Angaben der Autoren einige FHA mit cranio-kaudaler Ausrichtung, andere mit anteriorer Ausrichtung (Kettler et al. 2004).

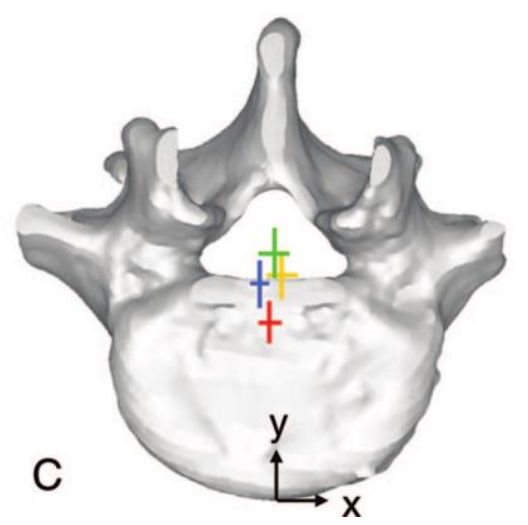

Abb. 13 Darstellung der HAM bei axialer Rotation in Takigawa et al. (2010). Sichtbar ist die Abhängigkeit des Schnittpunktes der HAM mit der Transversalebene von der Tatsache, ob eine Vorlast appliziert wurde oder nicht. So führt die Applikation einer Vorlast zu einer Anteriorverlagerung des Schnittpunktes (Vgl. grüne Kreuze und rote Kreuze).

Es wird jeweils nur eine Achsenposition, für verschiedene Variablen, dargestellt. Auf die blauen und roten Kreuze soll in der vorliegenden Arbeit nicht weiter eingegangen werden. (gelb Lokalisation der HAM ohne Vorlast, grün Lokalisation der HAM mit Vorlast, blau und rot Lokalisation der HAM mit TDR (total disc replacement), (aus Takigawa et al. 2010 S. 1163, mit Erlaubnis von Lipincott Williams and Wilkins/ Wolters Kluwer Health)

\section{typical pattern I typical pattern II}
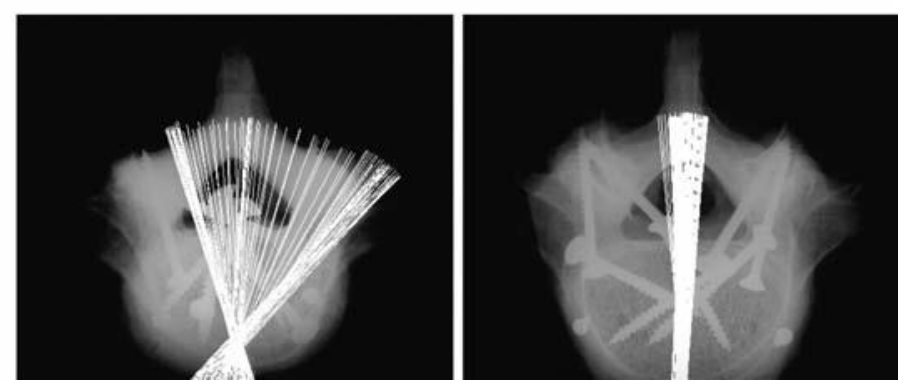

Abb. 14 Darstellung der Achsschar bei axialer Rotation in Kettler et al. (2004) in L4-L5 Segmenten. Die beiden Bilder zeigen verschiedene Verlaufsformen der Lage/ Ausrichtungen der FHA bei axialer Rotation, die auf interindividuelle Unterschiede der verwendeten Präparate zurückzuführen seien (Vergleich linke Abbildung, rechte Abildung), (aus Kettler et al. 2004, S. 556; mit freundlicher Erlaubnis von Springer Science and Business Media) 

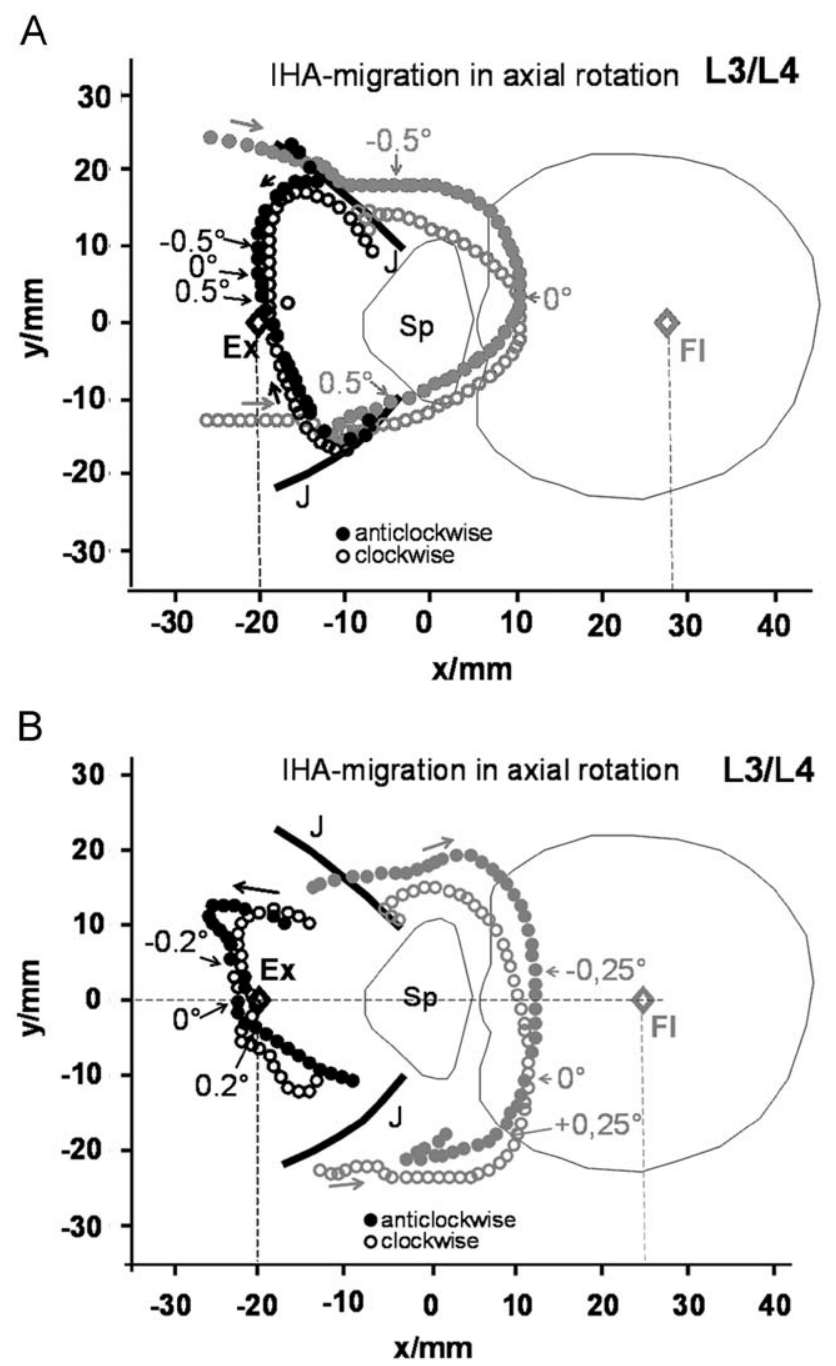

Abb. 15 Darstellung der Rastpolkurven in Wachowski et al. (2009a) bei axialer Rotation für die Segmente L3/L4. Die Rastpolkurve veräuft in Form einer Schleife in der Region des Spinalkanals (Sp).

Deutlich sichtbar wird der Einfluss der Position der Vorlast auf den Verlauf der Rastpolkurve. Der Verlauf der Rastpolkurve bei Dorsalverlagerung der Vorlast bei Bewegung ist durch schwarze Punkte, der Verlauf der Rastpolkurve bei Anteriorverlagerung der Vorlast durch graue Punke dargestellt. Die farblich ausgefüllten Punkte geben dabei die Bewegung von links nach rechts, die nicht ausgefüllten Punkte die Bewegung von rechts nach links an. (Wachowski et al. 2009a, S. 2288, mit Erlaubnis von Elsevier) 


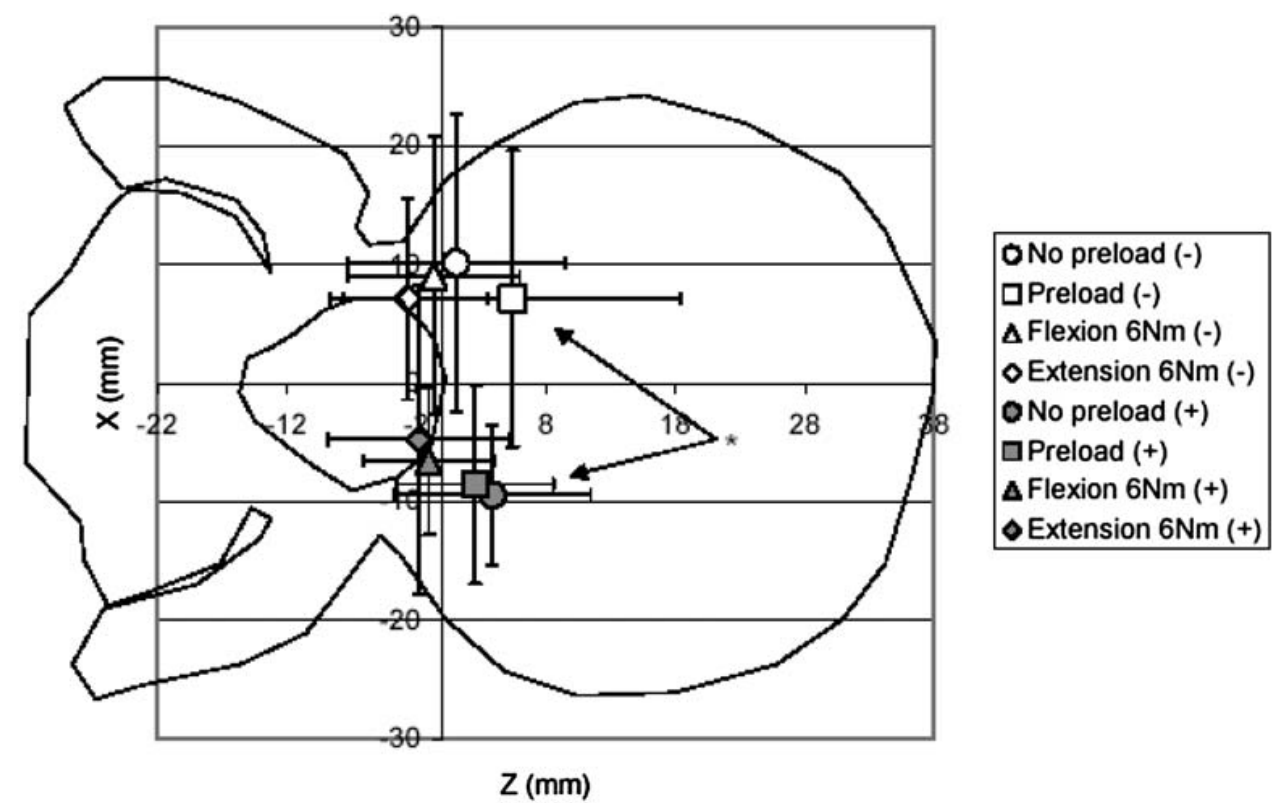

Abb. 16 Durchschnittlicher Schnittpunkt der HAM (für lumbale Segmente) mit Transversalebene in Haberl et al. (2004). Sichtbar wird der Einfluss der Vorlast auf die Lage der HAM.

(-) bedeutet axiale Rotation nach links, (+) bedeutet axiale Rotation nach rechts (aus Haberl et al. 2004, S.563; mit freundlicher Erlaubnis von Springer Science and Business Media).
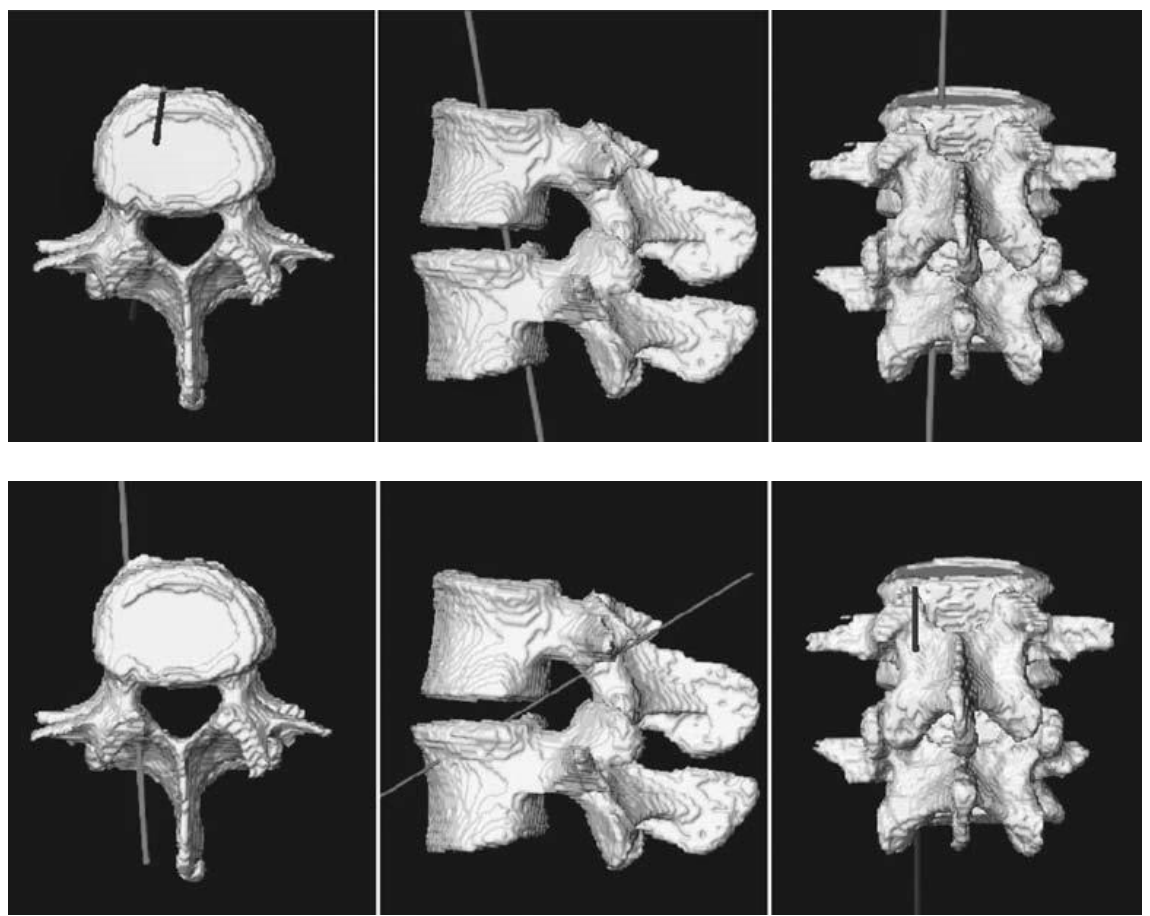

Abb. 17 Lage und Orientierung der HAM (für lumbale Segmente) in Haberl et al. (2004) für jewils zwei Positionen während der Bewegung (oberes Bild Linksrotation, unteres Bild Rechtsrotation), Darstellung in transversaler, sagittaler und posteriorer Ansicht (von links nach rechts). Sichtbar wird die unterschiedliche Orientierung der HAM bei verschiedenen Phasen der axialen Rotation (aus Haberl et al 2004, S. 563; mit freundlicher Erlaubnis von Springer Science and Business Media) 


\subsubsection{Lumbale Segmente laterale Beugung}

Bei Kettler et al. zeigten sich, wie auch schon für die anderen beiden Bewegungsrichtungen große interindividuelle Unterschiede bezüglich der Lage und Position der errechneten FHA im Segment L4-L5. Zum einen fanden sich Rotations/Schraubachsen, die parallel zueinander in einer anterior-posterioren Ausrichtung innerhalb der Horizontalebene den Wirbelkörper mittig durchliefen. Zum anderen zeigte sich eine starke Streuung der Achsschar innerhalb der Horizontalebene mit einem zentralen Kreuzungspunkt der Achsen im anterioren Drittel der Bandscheibe (Kettler et al. 2004) (s. Abb. 19).

Wachowski et al. gaben an, dass die IHA abhängig von der Position der Vorlast unterschiedliche Lokalisationen zeigte. Bei anterior positionierter Vorlast und Rotationen von $+/-2^{\circ}$ lag die IHA nahezu parallel zum Drehmoment, ohne zu wandern. Bei Rotationen über $+/-2^{\circ}$ wanderte die IHA jedoch kranial bzw. kaudal. Die IHA lag dabei im Bereich der Bandscheibe.

Bei Verlagerung der Vorlast nach posterior zeigte sich hingegen eine konstant zum Drehmoment parallel ausgerichtete IHA, die entgegengesetzt zur Bewegungsrichtung von einem Facettengelenk in Richtung des anderen wanderte und tendenziell im Bereich des Wirbelkörpers L4 lokalisiert war (s. Abb. 20) (Wachowski et al. 2009a).

Bei Takigawa et al. zeigte sich bei anteriorer Ansicht ein Kreuzungspunkt der HAM mit der Frontalebene zentral um den kaudalen Bereich des oberen Wirbelkörpers (L4) (s. Abb. 18). Bei Applikation einer Vorlast ( 400 N) zeigte sich eine kaudal Verlagerung der HAM in den Bereich der Bandscheibe. Die HAM war dabei nahezu parallel zum angelegten Drehmoment ausgerichtet. Die Applikation einer Vorlast hatte auf die Ausrichtung kaum Auswirkung (Takigawa et al. 2010).

Bei Zhu et al. lag die errechnete HAM im kaudalen Bereich des oberen Wirbelkörpers (L4) und war dabei annähernd parallel zum angelegten Drehmoment ausgerichtet. Die Angaben wurden bei Applikation einer Vorlast von $600 \mathrm{~N}$ gemacht (Zhu et al. 2007). Ähnliche Angaben für L3-L4 Segmente machten auch Niosi et al., jedoch für Versuche ohne Vorlast. Hier zeigten sich im Vergleich zu Zhu et al. nur leichte Unterschiede bezüglich der Orientierung (Niosi et al. 2006). 


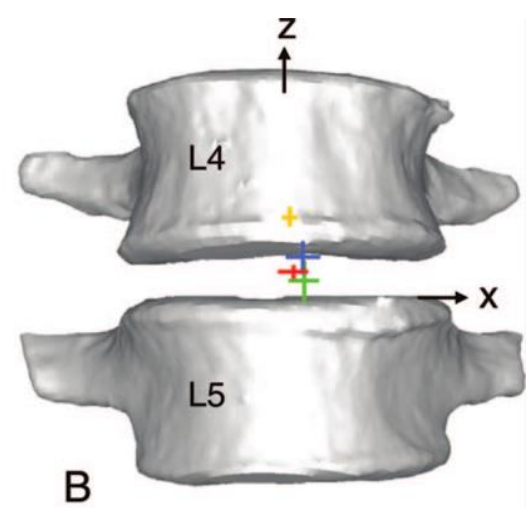

Abb. 18 Darstellung der HAM bei lateraler Beugung in Takigawa et al. (2010), für das Level L4/L5. Sichtbar wird der Einfluss der Vorlast auf die Lage der HAM. Es wird jeweils nur eine Achsenposition für verschiedene Variablen dargestellt. Auf die blauen und roten Kreuze soll an dieser Stelle nicht weiter eingegangen werden (gelb Lokalisation der HAM ohne Vorlast, grün Lokalisation der HAM mit Vorlast, blau und rot Lokalisation der HAM mit total disc replacement, TDR). (aus Takigawa et al. 2010, S. 1163; mit Erlaubnis von Lipincott Williams and Wilkins/ Wolters Kluwer Health).

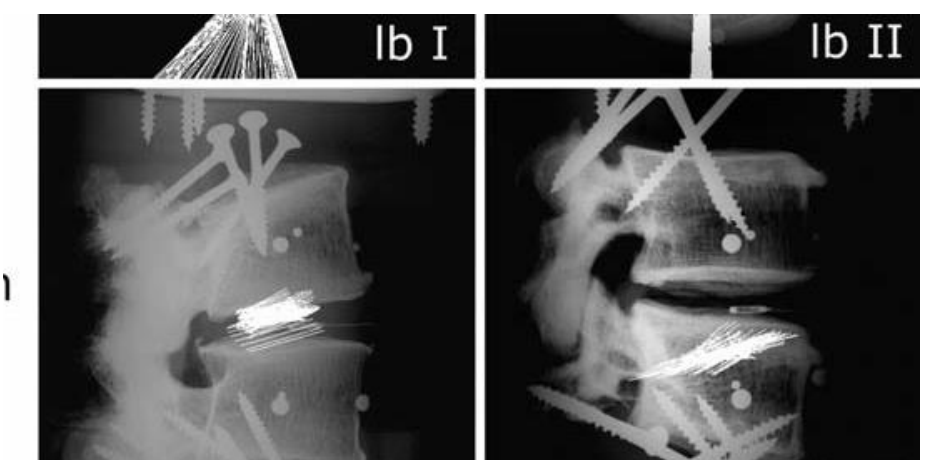

Abb. 19 Darstellung der Achsschar bei lateraler Beugung in Kettler et al. (2004) in L4-L5 Segmenten. Die Abbildungen zeigen, dass es interindividuelle Unterschiede zwischen verschiedenen Präparaten gibt (Vergleich linke Abbildung, rechte Abbildung). Sichtbar wird, die Auffächerung der Achsschar, die die wanderung der Schraubachse anzeigt (Kettler et al. 2004, S. 556; mit freundlicher Erlaubnis von Springer Science and Business Media). 


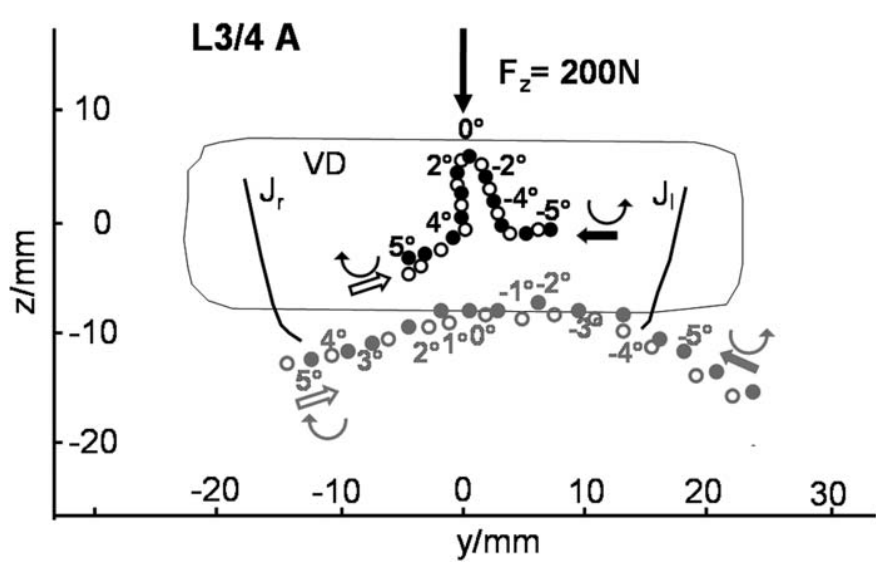

Abb. 20 Darstellung der Rastpolkurven in Wachowski et al. (2009a) bei lateraler Beugung. Sichtbar wird der Einfluss der Vorlast auf die Wanderung der Schraubachse. Die schwarz weißen Punkte geben dabei den Verlauf der Rastpolkurve bei Anteriorverlagerung, die grau weißen Punkte geben den Verlauf der Rastpolkurve bei Dorsalverlagerung der Vorlast wieder. Sichtbar wird, dass die Schraubachse nichts ortsfest ist. (aus Wachowski et al. 2009a, S. 2289, mit Erlaubnis von Elsevier).

\subsubsection{Lumbale Segmente Flexion-Extension}

McNally et al. gaben an, dass sich die Rotationsachse bei Flexionsmomenten bewege. In neutraler Stellung befinde sie sich in der Nähe des Zentrums der Bandscheibe im kaudalen Bereich des Zwischenwirbelraumes in den untersuchten Segmenten L3L4 und L4-L5. Bei Flexionsmomenten wanderte die IAR nach dorsal. Die Autoren gaben dabei an, dass sich die IAR sowohl in anterior-posterior Richtung als auch in kranio-kaudaler Richtung bewegte. Bezüglich der Ausrichtung der IAR fanden sich keine Aussagen (McNally et al. 2012).

Bozkus et al. berechneten die Lokalisationen der IAR in 0,5 - Intervallen, fassten die Positionen aller IAR jedoch zu einer Durchschnittsposition zusammen, die auf lateralen Röntgenbildern dargestellt wurde und sich während der Flexions/Extensionsbewegung in der Nähe des Zentrums des Wirbelkörpers L5, im kaudalen Bereich des Zwischenwirbelraumes befand. Die Angaben beziehen sich dabei auf das Segment L4-L5. Angaben zur Ausrichtung fanden sich nicht (Bozkus et al. 2010).

Ähnliche Angaben fanden sich auch bei anderen Autoren, die eine vergleichbare Lokalisation inrer berechneten HAM für L3-L4 (Niosi et al. 2006) und L4-L5 (Zhu et al. 2007, Takigawa et al. 2010) angaben. Hier lag die HAM etwas weiter anterior im Zwi- 
schenwirbelbereich und zeigte eine nahezu parallele Ausrichtung zur Frontal- und Transversalebene (Niosi et al. 2006, Zhu et al. 2007, Takigawa et al. 2010). Abbildung 21 zeigt eine mögliche Lokalisation der HAM nach Takigawa et al. (2010).

Kettler et al. unterschieden für Flexions-/Extensionsbewegungen in L4-L5 Segmenten zwei mögliche Verlaufsformen (s. Abb. 22) (Kettler et al. 2004).

Sengupta et al. bildeten den Verlauf der IAR auf lateralen Röntgenbildern ab und gaben für L3-L5 Segmente an, dass sich die IAR bei neutraler Stellung, nahe des Übergangs zwischen anterioren zwei Drittel und posteriorem Drittel des Zwischenwirbelraums und innerhalb der superioren Endplatte des unteren Wirbelkörpers befand. Während der Extensionsbewegung wanderte die IAR posterior in Richtung der Facettengelenke, während der Flexion bewegte sich die IAR wieder in Richtung Ausgangspunkt und weiter nach anterior-kranial in Richtung der unteren Deckplatte des oberen Wirbelkörpers. Die Gesamtbewegungsstrecke der IAR innerhalb der Sagittalebene betrug dabei $47 \mathrm{~mm}$. Zur Ausrichtung der Achse fanden sich auch hier keine Aussagen (Sengupta et al. 2011). Nägerl et al. stellten für L3/L4 bzw. L4/L5 Segmente eine zum angelegten Flexions-/Extensionsdrehmoment annähernd parallel ausgerichtete Schraubachse fest, die unter der Bandscheibe des Segmentes lag. Extensionsbewegung resultierte dabei in einer ausgeprägten Dorsalwanderung der Rastpolkurve in der Sagittalebene. Dorsalverlagerung der Vorlast führte zu einer generell weiter dorsal gelegenen Rastpolurve (Nägerl et al. 2009). Ähnliche Angaben fanden sich bei Wachowski et al. Auch hier lag die IHA parallel zum angelegten Drehmoment (Wachowski et al. 2009a, Wachowski et al. 2010a). Die Autoren beschrieben in der Sagittalebene eine Wanderung der IHA von einem Bereich dorsal des Spinalkanals in neutraler Stellung nach anterior in Richtung des Zentrums der Bandscheibe bei angelegtem Flexionsmoment. Während angelegtem Extensionsmoment wanderte die IHA dorsal bis zu den Facettengelenken. Bei Übergang von maximaler Extensionsstellung in die Flexionsbewegung zeigte die IHA einen kranialen Sprung innerhalb der Sagittalebene. Ähnliche Angaben fanden sich auch bei Wachowski et al. (2010b). Die Autoren gaben dabei an, dass die RPK eine Strecke von $30 \mathrm{~mm}$ zurücklegte (Wachowski et al. 2010b). Das Bewegungsausmaß war dabei bei Flexionsmomenten größer als bei Extensionsmomenten (Wachowski et al. 2009a). Abb. 23 zeigt exemplarisch die Darstellung einer RPK mit im obigen Text beschriebenen Verlauf. 
Detwiler et al. gaben keine genaue Lokalisation der errechneten axis of rotation an, Angaben zur Ausrichtung fanden sich ebenso nicht (Detwiler et al. 2003).

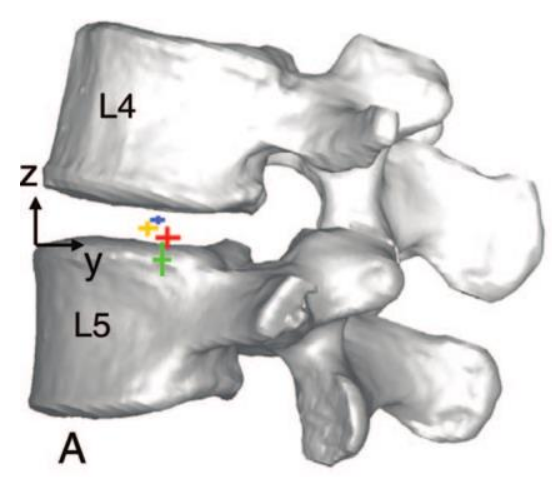

Abb. 21 Darstellung der HAM bei Flexion- Extension in Takigawa et al. (2010). Es wird jeweils nur eine Achsenposition, für verschiedene Variablen, dargestellt. Sichtbar wird der Unterschied zwischen der Lokalisation der HAM mit applizierter Vorlast (grün) und ohne (gelb). (blau und rot Lokalisation der HAM mit total disc replacement, TDR), (Takigawa et al. 2010, S. 1163; mit Erlaubnis von Lipincott Williams and Wilkins/ Wolters Kluwer Health)

\section{typical pattern I typical pattern II}
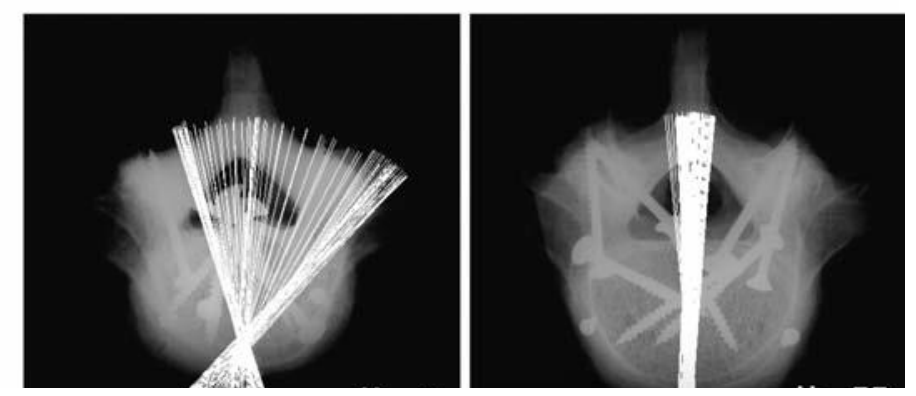

Abb. 22 Darstellung der Achsschar bei Flexion- Extension in Kettler et al. (2004) in L4-L5 Segmenten. Diese zeigt nach Angaben der Autoren zwei verschiedene Verlaufsformen (linke und rechte Abbildung). Sichtbar wird, dass die Schraubachse nicht an einem bestimmten Ort liegt, sondern während der Bewegung wandert (aus Kettler et al. 2004, S. 556; mit freundlicher Erlaubnis von Springer Science and Business Media) 


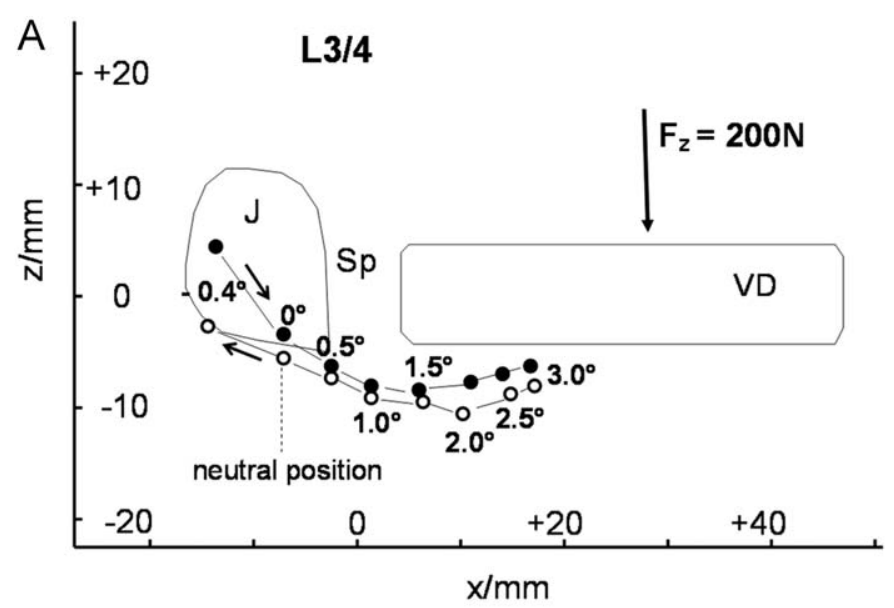

Abb. 23 Darstellung der Rastpolkurven in Wachowski et al. (2009a) bei Flexion- Extension. Sichtbar wird die Wanderung der, Schraubachse, die durch die Punkte dargestellt ist (Wachowski et al. 2009a, S. 2289; mit Erlaubnis von Elsevier).

\subsection{Angaben zum Bewegungsausmaß und Gelenkkräften in den untersuchten Studien}

\subsubsection{Bewegungsausmaß (ROM)}

Da das ROM aus physikalisch-kinematischer Sicht keine Rückschlüsse auf die Bewegungsstruktur gibt (s. Kapitel 1.5.3), soll darauf gemäß der Zielsetzung der vorliegenden Studie nur kurz eingegangen werden. Bis auf wenige Ausnahmen (Haher et al. 1991, Haher et al. 1992, Cripton et al. 2001, Rousseau et al. 2006a) ermittelten alle Autoren das ROM. Rousseau et al. und Haher et al. gaben das ROM vor, indem sie das jeweilige Präparat gezielt in $3^{\circ}$ und $6^{\circ}$ Flexions-/Extensions-/ bzw. laterale Beugungsstellungen brachten (Rousseau et al. 2006a, Rousseau et al. 2006b) oder $5^{\circ}$ bzw. $10^{\circ}$ axial rotieren ließen (Haher et al. 1992).

Einige Autoren gaben an, dass die Vorlastgröße bzw. Vorlastposition (Haberl et al. 2004, Wachowski et al. 2009a) einen Einfluss auf das ROM hat. Haberl et al. gaben an, dass bei angelegter axialer Rotationsbewegung und bei Anteriorveralgerung der Vorlast das ROM signifikant kleiner war, als bei Versuchen ohne Vorlast. Wachowski et al. fanden bei Posteriorveralgerung der Vorlast kleinere Werte für das ROM bei axialer Rotation in lumbalen Segmenten als bei Anteriorverlagerung der Vorlast (Wachowski et al. 2009a). Ähnlich Angaben für Flexions-Extensionsbewegungen fanden sich bei Nägerl et al. (Nägerl et al. 2009). Niosi et al. (2006) errechneten für alle drei Bewegungsrichtungen ein geringfügig verringertes ROM bei Versuchen mit follo- 
wer preload $(600 \mathrm{~N})$ im Vergleich zu Versuchen ohne follower preload.

Einige Autoren nahmen eine weitere Unterteilung des ROM in eine neutrale Zone (NZ) (Kettler et al. 2004, Niosi et al. 2006, Zhu et al. 2007, Zhu et al. 2012) und elastische Zone (EZ) (Oxland et al. 1994, Broc et al. 1997, Chen et al. 2001, Detwiler et al. 2003) bzw. lax zone (LZ) und stiff zone (SZ) vor (Naderi et al. 1998, Crawford et al. 2002, Cagli et al. 2004, Bozkus et al. 2010, Lazaro et al. 2010, Brasiliense et al. 2011). NZ bzw. LZ kennzeichnen dabei den Bereich, in welchem dem Segment ein geringer Widerstand durch die umliegenden anatomischen Strukturen bei Bewegung entgegengesetzt wird (Crawford et al. 1998). In der plastischen Zone wird das Präparat hingegen irreversibel geschädigt. Die EZ bzw. SZ kennzeichnen dabei den Bereich steigenden Widerstands und steigender Steifigkeit. Ein Anwachsen bspw. der NZ könne demnach auf eine sinkende Steifigkeit und somit auf Instabilitäten des Segmentes hindeuten (Panjabi 1992).

Nach Panjabi et al. kennzeichnet die NZ dabei das Bewegungsausmaß zwischen initialer, neutraler Position des Segmentes vor Testdurchlauf und dem Bewegungsausmaß, in welches das Segment nach einigen Testdurchläufen mit jeweils maximal angelegtem Drehmoment zurückkehrt, nachdem das Drehmoment wieder auf $0 \mathrm{Nm}$ reduziert wurde (Panjabi 1992). Der weitere Bereich bis zum vollen ROM stellt die EZ dar (Panjabi 1992).

Die lax zone unterscheidet sich von der neutralen Zone nach Crawford et al. dadurch, dass hier minimaler ligamentärer Widerstand vorherrschen solle, wohingegen in der neutralen Zone eine geringe Gelenkreibung vorherrsche (Crawford et al. 1998). Broc et al. beschrieben hingegen auch die NZ als den Bereich, in dem geringer ligamentärer Widerstand vorliege, der dann in der EZ ansteige (Broc et al. 1997). Ein Äquivalent zur elastischen Zone stellt die stiff zone dar (Crawford et al. 1998) (s. auch Kapitel 1.3). Crawford et al. schlagen dazu eine Methode vor, wie die stiff zone zu bestimmen sei: Bei gleichmäßig steigendem Bewegungsausmaß steigt die Drehmomentgröße immer stärker an, was die Steifigkeit des Segmentes angeben solle. Aus dieser grafischen Darstellung können nun Rückschlüße auf den Übergang der lax zone in die stiff zone und umgekehrt gefolgert werden.

Bozkus et al. gaben an, dass die lax Zone mit der neutralen Zone vergleichbar sei, aber eine höhere Reproduzierbarkeit aufweise (Bozkus et al. 2010). 
Allgemein diente die Ermittlung der Parameter NZ, EZ bzw. LZ und SZ einer genaueren Beurteilung des ROM in Bereiche geringer Steifigkeit (NZ, LZ) bzw. Bereiche steigender Steifigkeit (EZ, SZ). Die Werte der einzelnen Parameter wurden dabei auch verwendet, um sie z. B. vor und nach Bandscheibenimplantation (z. B. Kettler et al. 2004 und andere), Resektion anatomischer Strukturen (z. B. Broc et al. 1997, Detwiler et al. 2003, Brasiliense et al. 2011), Fixierungsmethoden (z. B. Dickman et al. 1996, Naderi et al. 1998 und andere) mit Werten aus dem intakten Zustand des Präparates zu vergleichen.

\subsubsection{Gelenkkräfte}

Einige Autoren untersuchten die Gelenkkräfte, die während des Testdurchlaufes entstanden. Dadurch sollte die Belastung benachbarter Segmente untersucht werden, da bekannt ist, dass nach Implantation oder Fusion häufig eine Überbelastung benachbarter Segmente vorliegt (Beutler et al. 2012). Gelenkkräfte wurden dabei über Sensoren gemessen, die durch Eröffnung der jeweiligen Gelenkkapsel in das zu untersuchende Gelenkfläche eingebracht wurde. Einige Autoren gaben hierzu an, dass es zu bewegungsverändernden Eingriffen gekommen sei und testeten die zur Verfügung stehenden Präparate daher zum Teil mit und ohne Gelenkkraftsensoren (Takigawa et al. 2010).

Rousseau et al. maßen die Gelenkkräfte, indem sie Sensoren mit einer Dicke von $0,2 \mathrm{~mm}$ an der Gelenkfläche anbrachten. Nach Angaben der Autoren nahmen die Sensoren dabei bis zu der Hälfte der gesamten Gelenkfläche ein. Zum Einbringen der Sensoren mussten die Gelenkkapseln eröffnet werden (Rousseau et al. 2006a, Rousseau et al. 2006b). Lazaro et al. verwendeten hingegen Dehnmessstreifen, die an oder in der Nähe der Facettengelenke befestigt waren (Lazaro et al. 2010). Ob dabei die Gelenkkapseln eröffnet wurden, wurde von den Autoren nicht angegeben. Bozkus et al. maßen die Kräfte zweier Dehnmesstreifen, die an in das Segment eingebrachte Pedikelschrauben befestigt waren (Bozkus et al. 2010). Cripton et al. maBen die Dehnung der anatomischen Strukturen in $\mu \mathrm{m} / \mathrm{m}$ innerhalb der Gelenke über sogenannte strain rossettes, die an den Gelenkflächen und im anterioren Bereich des Wirbelkörpers angebracht waren (Cripton et al. 2001). Takigawa et al. verwendeten zur Gelenkkraftmessung piezoresistive Sensoren, mit einer Dicke von 0,2 mm. Die Sensoren wurden in 7 der 14 verwendeten Präparate eingesetzt. Dazu mussten die Gelenkkapseln beschädigt werden. Die anderen 7 Präparate blieben unverändert. Somit sollte ein Vergleich des Einflusses der Sensoren auf die Kinematik des Seg- 
mentes beurteilt werden können. Das ROM und die HAM zeigten dabei nach Angaben der Autoren nur leichte, nicht signifikante Unterschiede (Takigawa et al. 2010). 


\section{Diskussion}

\subsection{Zielstellungen der untersuchten Studien}

Die systematische Unterteilung der zu untersuchenden Arbeiten zeigte, dass erhebliche Unterschiede in den Zielsetzungen der Studien bestanden (s. Kapitel 3.1).

So wurde gezielt das kinematische Verhalten der Segmente nach Implantateinsatz oder nach Resektion anatomischer Strukturen untersucht. Zusätzlich ist zu bedenken, dass auch unterschiedliche Segmente untersucht wurden. Für die vorliegende Analyse bedeutet dies, dass die kinematischen Ergebnisse der einzelnen Studien nur bedingt direkt vergleichbar sind. So ist beispielsweise der Vergleich der Migration einer Schraubachse in lumbalen Segmenten (z. B. Mansour et al. 2004) nicht mit der von zervikalen Segmenten (z. B. Naderi et al. 1998) vergleichbar. Vielmehr sollen die Studien bezüglich ihrer Methodik, also der Umsetzung der in Kapitel 1.3-1.5 beschriebenen Grundlagen und der in Kapitel 1.6 gestellten Forderungen analysiert werden.

\subsection{Testapparatur und Drehmomentapplikation}

Den jeweils verwendeten Testapparaturen kommt eine hohe Bedeutung zu, da ihre technische Konstruktion im Wesentlichen die Anzahl der ermöglichten Freiheitsgrade bedingt. Des Weiteren müssen durch sie die drei heuristisch vorgegebenen Bewegungsrichtungen (Flexion/Extension, laterale Beugung, axiale Rotation) ermöglicht werden.

Wilke et al. veröffentlichten 1998 eine Leitlinie für die In-vitro-Untersuchung von Bandscheibenimplantaten. Diese Leitlinie wurde im Rahmen der von der Gesellschaft für Wirbelsäulenchirurgie (heute „Deutsche Wirbelsäulengesellschaft") durchgeführten Workshops erarbeitet. Ziel dieser Leitlinie war es, Testmethodiken zu vereinheitlichen, um somit eine größere Vergleichbarkeit der Testergebnisse verschiedener Studien zu erhalten (Wilke et al 1998a). Da diese Leitlinie nach Ansicht des Autors der vorliegenden Analyse jedoch auch für In-vitro-Testverfahren von intakten Segmenten Gültigkeit haben sollte, wird sie in der folgenden Studie auch zur Diskussion von Testverfahren mit Wirbelsäulensegmenten ohne Bandscheibenimplantate herangezogen.

Wilke et al. fordern, dass die Testapparatur einem Wirbelkörper 6 DOF im Raum ermöglichen solle (Wilke et al. 1998a) (s.a. Kap. 1.5.2). Die Analyse der Studien zeigte jedoch, dass nur wenige Autoren angaben, eine Apparatur verwendet zu haben, die diesen Anforderungen gerecht wurden (s. Tab. 1, S. 33) (Grassmann et al. 1998, 
Mansour et al. 2004, Nägerl et al. 2009, Sengupta et al. 2011, Wachowski et al. 2007, Wachowski et al. 2009a, Wachowski et al. 2009b, Wachowski et al. 2010, Wachowski et al. 2010b, Wachowski et al. 2013). Wesentliches Merkmal der verwendeten Apparaturen war hierbei, dass die zur Drehmomentapplikation genutzten Elemente (s. Kap. 3.2) nicht in direkter Verbindung mit den zu testenden Segmenten standen und somit der durch das jeweilige Präparat bestimmten Bewegung folgten.

In den meisten Fällen wurden diesbezüglich entweder keine Angaben gemacht oder die Apparaturen konnten die Anforderung, eine rückwirkungsfreie Bewegung zu generieren nicht erfüllen (s. Tab. 1, S. 33), was jedoch als wesentliche Anforderung an eine korrekte Aufzeichnung der Segmentkinematik formuliert wurde (s. Kap. 1.5.2). Dies stellt die Bedeutung der Messergebnisse der jeweiligen Studien in Frage:

Zhu et al. und Niosi et al. verwendeten eine Testapparatur mit der nach Angaben der Autoren theoretisch ungeführte Bewegungen möglich seien (Niosi et al. 2006, (Zhu et al. 2007, Zhu et al. 2012). Unter einer ungeführten Bewegung wird dabei die natürliche Bewegung des Testobjektes ohne Beeinflussung durch die Testapparatur verstanden (Crawford et al. 1995). In einer Studie von Goertzen et al. (2004), in der die verwendete Testapparatur näher beschrieben wurde, zeigte sich jedoch, dass dem Präparat lediglich 5 DOF ermöglicht wurden. Somit kann nicht von einer ungeführten Bewegung ausgegangen werden.

Grassmann et al. (1998) untersuchten in einer Studie gezielt den Einfluss eines Testdurchlaufes mit zwei Apparaturen, die entweder eine ungeführte oder geführte Bewegung ermöglichten. Im Falle der geführten Bewegung wurde die Rotationsachse durch den Aufbau der Apparatur bestimmt und die Rotation des Präparates gemessen. Es wurden fünf verschiedene Positionen der Rotationsachse festgelegt und der Rotationswinkel des Präparates gemessen. Dabei zeigte sich ein signifikanter Unterschied zwischen minimalem und maximalem Rotationswinkel in Abhängigkeit von der durch die Testapparatur bedingten Rotationsachse. Die Autoren konnten damit zeigen, dass das Bewegungsverhalten des Präparates in direktem Zusammenhang mit dem Aufbau der Testapparatur steht (Grassmann et al. 1998). Die Forderung, eine Apparatur zu verwenden, die ungeführte Bewegungen in 6 DOF erlaubt, konnte damit untermauert werden. Um physiologische Testbedingungen zu erhalten, sollten daher immer valide Angaben über die Anzahl der ermöglichten Freiheitsgrade gemacht werden. Wird dieser Anforderung, wie in einigen Fällen, nicht Rechnung getragen 
(Cusick et al. 1988, Haher et al. 1991, Haher et al. 1992, Oxland et al. 1994, Dickman et al. 1996, Broc et al. 1997, (Naderi et al. 1998, Chen et al. 2001, Cripton et al. 2001, Crawford et al. 2002, Detwiler et al. 2003, Cagli et al. 2004, Haberl et al. 2004, Kettler et al. 2004, Molnar et al. 2006, Bozkus et al. 2010, Dugailly et al. 2010, Takigawa et al. 2010, Lazaro et al. 2010, Dugailly et al. 2011, McNally et al. 2012, Dugailly et al. 2013, Brasiliense et al. 2011), so kann nicht sicher von einer physiologischen Bewegung des Präparates ausgegangen werden, was entsprechend auch die Messergebnisse in Frage stellt.

In einigen der genannten Studien wird jedoch durch die Beschreibung der Versuchsapparatur offensichtlich, dass keine 6 DOF ermöglicht wurden:

So kann die von Dugailly et al. $(2010,2011,2013)$ in mehreren Versuchen applizierte Bewegung keine physiologischen Verhältnisse simulieren. Die Autoren gaben hierzu an, dass sich die zur Generierung der Bewegung erforderlichen Stangen in einem Wirbelkörper über dem eigentlichen Testsegment befanden und daher im Testsegment eine physiologische Bewegung vorliege. Dabei ist es jedoch möglich, dass der obere Wirbelkörper der Stellung der von Hand bewegten Stangen folgt und sich diese unphysiologische Bewegung auch auf das eigentliche Testsegment fortpflanzt. Es wird also keineswegs die Forderung einer Bewegung in 6 DOF erfüllt. Ein weiterer Nachteil ist, dass keine angreifenden Drehmomentgrößen gemessen werden können. Die auf das Segment einwirkenden Parameter sind daher weitgehend unbekannt und werden nur manuell durch den Untersucher vorgegeben, was eine reproduzierbare Messmethodik nicht zulässt.

Crawford et al. stellten eine Apparatur vor (Crawford et al. 1995), die nach Angaben der Autoren eine kosteneffektive Installation versprach und mit der physiologische Bewegungen generiert werden könnten. Entsprechend wurde die Apparatur von zahlreichen Autoren genutzt (Dickman et al. 1996, Broc et al. 1997, Naderi et al. 1998, Chen et al. 2001, Detwiler et al. 2003, Cagli et al. 2004, Bozkus et al. 2010, Lazaro et al. 2010, Brasiliense et al. 2011). Crawford et al. erkannten, dass auf das Präparat nicht jederzeit nur das angelegte Drehmoment wirkte, sondern auch ein Artefaktmoment von $0,13 \mathrm{Nm}$ in lateraler Beugungsrichtung bei angelegtem Flexionsmoment von $1,5 \mathrm{Nm}$ vorlag. Daher musste, wie in Kapitel 3.2 beschrieben, eine ständige, manuelle Nachjustierung des Kabelverlaufs erfolgen. Dies stellt eine erhebliche Limiterung der jeweiligen Testdurchläufe dar, wie auch Caravaggi in einer Beschreibung der Testap- 
paratur feststellten (Caravaggi et al. 2013). Zum einen ist dadurch keine kontinuierliche Bewegung möglich, da der Testdurchlauf für die Justierung der Kabelführungen unterbrochen werden muss. Zum anderen ist der erforderliche horizontale Verlauf der Kabel manuell kaum exakt einzustellen. Die Autoren gaben daher an, dass mit der vorgestellten Apparatur nur eine nahezu ungeführte Bewegung möglich sei (Crawford et al. 1995), obwohl zunächst von den Autoren der Studie selbst, wie auch von anderen Autoren, die die beschriebene Apparatur verwendeten, angegeben wurde, rückwirkungsfreie, den Bewegungscharakter der Präparate nicht beeinflussende Drehmomente zu generieren (Dickman et al. 1996, Broc et al. 1997, Detwiler et al. 2003, Cagli et al. 2004, Bozkus et al. 2010, Lazaro et al. 2010, Brasiliense et al. 2011). Dies ist jedoch aus den genannten Gründen nicht der Fall, so dass auch von einer Bewegung in 6 DOF nicht ausgegangen werden kann.

McNally et al. (2012) und Cusick et al. (1988) nutzten die gleichen Bestandteile der jeweiligen Apparatur zur Befestigung des Präparates, Applikation des Drehmoments und der Vorlast. Über Befestigungsplatten, die die Präparate am oberen und unteren Ende in der Apparatur hielten, wirkten unweigerlich Kräfte auf das Präparat (s. Abb. 10, S. 37). Hierbei besteht jedoch der Nachteil, dass Bewegungen im Sinne der coupled motion von der Testapparatur unterdrückt wurden. McNally et al. (2012) führten dies sogar bewusst herbei, da die Bewegungen, nach Ansicht der Autoren, im Sinne der coupled motion ohnehin nur gering seien. Dies stellt jedoch eine erhebliche Vereinfachung des Testdurchlaufes dar, die keine 6 DOF zulässt und somit nicht den physiologischen Gegebenheiten entspricht. Zudem ist festzustellen, dass sich das Segment in Folge der coupled motion unter physiologischen Bedingungen beispielsweise in vertikaler Richtung bewegt und daraus, durch die starre Anordnung der Befestigungsplatten eine erhöhte Kompression des Präparates und somit eine erhöhte Vorlast resultieren würden. Dies stellten auch Grassmann et al. (1998) in einem Versuch mit einer vergleichbaren Apparatur fest. Auch bei Haher et al. (1991) und Rousseau et al. (2006a, 2006b) ist durch die starre Anordnung des oberen und unteren Teils der Testapparatur von zusätzlichen internen Kompressionskräften auszugehen. Die von Rousseau et al. verwendete Apparatur ermöglichte dem Präparat dabei 4 DOF (Rousseau et al. 2006a, Rousseau et al. 2006b). Die Autoren gaben dies entsprechend als Limitierung der Studie an, die unter Umständen die Messergebnisse beeinflusst haben könnte (Rousseau et al. 2006a). Die Tatsache, dass die Apparatur in einem Testdurchlauf verwendet wurde, der ein Bandscheibenimplanatat mit 5 DOF 
ermöglichen sollte, macht die Limitierung des angewandten Testverfahrens deutlich. Denn die Testapparatur erlaubte dem Testobjekt nur 4 DOF. Dies macht die Limitierung des Testverfahrens eindrucksvoll deutlich. Rousseau et al. generierten dabei die Bewegung über eine vertikale Belastung und Keile. Dabei resultierten Stellungen von $3^{\circ}$ und $6^{\circ}$ (Rousseau et al. 2006a, Rousseau et al. 2006b). Wenn auch das Rotationsausmaß noch in physiologischen Größen liegt, ist dennoch fragwürdig, ob es sich bei der Stellung des Testsegmentes um eine physiologische Bewegung handelt oder das Präparat lediglich einer durch den Keil vorgegebenen Stellung folgt. Auch Erkenntnisse über die Größe des angelegten Drehmoments waren somit nicht möglich. Auch Haher et al. (1991) generierten die Bewegung über einen Keil und vertikale Belastung, so dass hier die gleichen Kritikpunkte gelten müssen.

Schlussfolgernd muss festgestellt werden, dass die Art der Befestigung des Präparates und Applikation des Drehmomentes zwischen den Studien stark variiert. So stehen sich Untersuchungen mit technisch aufwendigen Testapparaturen (z. B. Takigawa et al. 2010, Wachowski et al. 2013 und andere), in denen rückwirkungsfreie Bewegungen generiert werden können, Verfahren gegenüber, in denen die Segmente in sehr einfachen Verfahren manuell durch den Untersucher bewegt werden (z. B. Dugailly et al. 2010).

Einen völlig anderen Ansatz verfolgten Schulze et al. (2012). Hier war die direkte Kontrolle der applizierten Bewegung Ziel des Testverfahrens. Die erfolgten Bewegungen haben damit eine hohe Reproduzierbarkeit. Jedoch müssen die applizierten Bewegungen den physiologischen Verhältnissen entsprechen, was jedoch nur eingeschränkt möglich ist, da zwischen den verschiedenen Präparaten interindividuelle Unterschiede bestehen, die in diesen Versuchen vernachlässigt wurden. Die Messergebnisse sind jedoch besser vergleichbar, da genaue Kenntnis über die angelegten Parameter vorliegt. Den individuellen Bewegungsstrukturen des Präparates wird dabei jedoch nicht gefolgt, wodurch die Ermittlung einer Schraub-/Rotationsachse wenig Aufschluss über die kinematischen Bewegungsstrukturen der Wirbelsäule bietet.

In einigen Fällen kann auch nach Analyse der Angaben zum Aufbau der jeweiligen Testapparatur nicht festgestellt werden, ob dem Testobjekt eine ungeführte Bewegung in 6 DOF ermöglicht wurde (Haher et al. 1992, Oxland et al. 1994, Cripton et al. 2001, Haberl et al. 2004, Kettler et al. 2004, Molnar et al. 2006). Somit sind auch die Messergebnisse der Studien kritisch zu hinterfragen. In den Fällen in denen den 
Segmenten nachweislich keine 6 Freiheitsgrade ermöglicht wurden, können bezüglich der Segmentkinematik, auch keine validen Messergebnisse erreicht werden: (Cusick et al. 1988, Haher et al. 1991, Dickman et al.1996, Broc et al.1997, Naderi et al. 1998, Chen et al. 2001, Crawford et al. 2002, Detwiler et al. 2003, Cagli et al. 2004, Niosi et al. 2006, Rousseau et al. 2006a, Rousseau et al. 2006b, Zhu et al. 2007, Bozkus et al. 2010, Dugailly et al 2010, Lazaro et al. 2010, Brasiliense et al. 2011, Dugailly et al. 2011, McNally et al. 2012, Zhu et al. 2012, Dugailly et al. 2013).

\section{Drehmomentgröße}

Hinsichtlich der angelegten Drehmomentgröße konntef in der vorliegenden Arbeit gezeigt werden, dass erhebliche Unterschiede zwischen den in den Studien angelegten Drehmomentgrößen bestehen (s. Tab. 1). Dies hat aus zwei Gründen grundlegende Bedeutung: Zum einen stellen zu hohe oder zu niedrige Werte unphysiologische Verhältnisse dar, zum anderen ist bei unterschiedlichen Drehmomentgrößen die Vergleichbarkeit der Studien untereinander erheblich eingeschränkt.

Wilke et al. (1998a) schlugen in ihrer Leitlinie für In-vitro-Untersuchungen von Bandscheibenimplantaten vor, dass in lumbalen Segmenten $+/-7,5 \mathrm{Nm}$, in thorakalen Segmenten $+/-5 \mathrm{Nm}$ und in zervikalen Segmenten $+/-1 \mathrm{Nm}$ angelegt werden sollten (Wilke et al 1998a).

Nach Analyse der in den jeweiligen Studien angelegten Drehmomentgrößen zeigte sich, dass in zervikalen Segmenten eine deutlich geringere Drehmomentgröße angelegt wurde als in lumbalen bzw. thorakalen Segmenten. In lumbalen Segmenten zeigten sich hingegen die größten Abweichungen mit Drehmomentgrößen von +/-5 Nm (Detwiler et al. 2003) bis $40 \mathrm{Nm}$ (McNally et al. 2012) jeweils für Flexionsbewegungen. Crawford et al. gaben hingegen an, dass bereits $+/-10 \mathrm{Nm}$ für lumbale Segmente einen annähernden Maximalwert der Drehmomentgröße gemessen an physiologischen Werten darstellten (Crawford et al. 2002). Höhere Werte fanden sich bei Invivo-Messungen von Adams und Dolan, die beispielsweise für das Aufheben eines Stiftes etwa 15-20 Nm gemessen haben (Adams und Dolan 1991).

Für zervikale und thorakale Segmente fanden sich in der Literatur nur wenige Vorschläge über die Größe des zu applizierenden Drehmomentes. Panjabi et al. schlugen für zervikale Segmente eine Drehmomentgröße von +/-1,0 Nm vor (Panjabi et al. 1976). Ähnliche Angaben fanden sich bei Wilke et al. (Wilke et al 1998a). Die in den analysierten Studien angelegten Drehmomentgrößen für zervikale Segmente gingen 
nur geringfügig über diese in der Literatur empfohlenen Maximalwerte hinaus. In thorakalen Segmenten schlugen Wilke et al. Drehmomentgrößen von $+/-5 \mathrm{Nm}$ vor (Wilke et al. 1998a), was in etwa den angelegten Werten in den untersuchten Studien entspricht (s. Tab. 1).

Bezüglich der Größe des Drehmomentes ist zu beachten, dass bei zu kleinen Werten das Segment nicht das volle Bewegungsausmaß erreicht (Wilke et al. 1998a). Adams und Dolan (1991) gaben hierzu an, dass die in vivo erreichten Maximalwerte bezüglich des Drehmomentes nur 35\% der Größe entsprächen, die In-vitro zu Schäden der Wirbelsäule führen könnten. Wenngleich Patwardhan et al. (2012) angaben, dass bei mehreren Versuchswiederholungen mit zervikalen Segmenten und einem angelegten Drehmoment von über +/-1,5 Nm das Risiko für Verletzungen des Weichgewebes zunehme, sollten die in den untersuchten Studien angelegten Drehmomentgrößen (s. Tab. 1) nicht als unphysiologisch hoch angesehen werden, da auch bei Werten von bis zu +/- $30 \mathrm{Nm}$ (Wachowski et al. 2009a) oder $40 \mathrm{Nm}$ (McNally et al. 2012) nicht von Schädigungen der Präparate berichtet wurde.

Die Analyse der Studien zeigte jedoch, dass keine einheitlichen Drehmomentgrößen appliziert wurden. In zervikalen Segmenten wurden geringere Drehmomentgrößen angelegt als in lumbalen und thorakalen. Für lumbale Segmente wurden tendenziell +/- 7.5 Nm als geeignet angesehen und von zahlreichen Autoren angelegt (s. Tab. 1). Um eine gute Vergleichbarkeit zwischen den Studien erzeugen zu können, sollten für die Größe des Drehmomentes einheitliche Werte angelegt werden, was jedoch häufig nicht der Fall ist (s. Tab. 1).

Die Beschreibung des Aufbaus der verwendeten Testapparaturen konnte zeigen, dass das Drehmoment in allen Fällen an einem Ende des Testobjektes appliziert wurde. Wurden dabei polysegmentale Präparate verwendet, so befand sich oftmals zwischen dem Wirbelkörper, an dem das Drehmoment appliziert wurde und dem eigentlichen Testsegment andere Segmente, über die das Drehmoment weiter bis zum eigentlichen Testsegment geleitet wurde. Das resultierende Drehmoment im Testsegment sollte nun erwartungsgemäß kleiner und möglicherweise in seiner Ausrichtung verändert sein. Damit erhöht sich die Zahl der Variablen. Dies verhält sich bei monosegmentalen Präparaten anders, da hier das angelegte Drehmoment entsprechend direkt auf das eigentliche Testsegment wirkt. Daher muss bei Angaben zur angelegten Drehmomentgröße auch berücksichtigt werden, aus wie vielen Segmenten das 
Präparat bestand.

Einige Autoren legten während des Testdurchlaufes Unterbrechungen ein, um so dem Effekt des Creeps Rechnung zu tragen (s. Tab. 1, S 33). Nach der Unterbrechung wurde der Testdurchlauf dann mit einem höheren Drehmoment fortgeführt. Der Effekt des Creeps wird auf die viskoelastischen Eigenschaften der Bandscheibe zurückgeführt. Hierbei kann sich das Präparat an die auf sie einwirkenden Parameter anpassen, so dass durch Abnehmen der initialen Steifigkeit bzw. viskoelastischer Effekte ein höheres Bewegungsausmaß zu erwarten ist. Jedoch ist zu hinterfragen, inwiefern in vivo tatsächlich diese Form einer unkontinuierlichen, sprunghaften Bewegung vorliegt, worauf auch Adams in einer Studie hinwies (Adams 1995). Soll das Ziel der Untersuchung die Ermittlung des maximalen Bewegungsausmaßes sein, so können durch die Unterbrechungen womöglich höhere Werte erreicht werden, als ohne Unterbrechungen. Jedoch sollte- zur Ermittlung dynamischer Bewegungsverhältnissebesser auf kontinuierliche Bewegungen zurückgegriffen werden, da diese eher dem natürlichen Bewegungsmuster entsprechen. Die schrittweise Applikation des Drehmomentes, wie von zahlreichen Autoren angewandt (s. Tab. 1), ist entsprechend für die Ermittlung der physiologischen Segmentkinematik nicht sinnvoll, da sie auch nicht der physiologischen Bewegung entspricht.

In einigen Fällen wurde auch die Geschwindigkeit der Bewegung des Segmentes angegeben (s. Tab. 1). Adams gab an, dass die Steifigkeit des Segmentes zunimmt, je schneller eine Bewegung erfolgt und empfahl daher, dass sich die Applikationsrate in physiologischen Bereichen bewegen sollte. Nach Angaben des Autors sollten für eine Beugungsbewegung 0,2 bis $5 \mathrm{~s}$ angenommen werden (Adams 1995). Nimmt man für eine Flexions-/Extensionsbewegung in lumbalen Segmenten ein Bewegungsausmaß von $10^{\circ}$ an (z. B. Bozkus et al. 2010 bei $+/-7,5 \mathrm{Nm}$ ), bedeutet dies eine Applikationsrate von $2 \% \mathrm{~s}$ für einen Zeitraum von $5 \mathrm{~s}$. Wilke et al. konnten hierzu zeigen, dass Rotationsgeschwindigkeiten des Segmentes von $0,6 \% \mathrm{~s}$ bis $5,1 \% \mathrm{~s}$ keinen signifikanten Einfluss auf das Bewegungsausmaß haben (Wilke et al. 1998b). Größtenteils werden diese Werte auch von den Autoren verwendet (s. Tab. 1). Lediglich Naderi et al. (1998) generierten mit einer Applikationsrate von 10\% s eine deutlich höhere Rotationsgeschwindigkeit für zervikale Segmente. Dies könne nach Wilke et al. (1998a) einer erhöhten Trägheit des gesamten Systems führen.

Zusammenfassend ist also festzustellen, dass bezüglich der Drehmomentgröße, der 
resultierenden Drehgeschwindigkeit und der Frage, ob das Drehmoment kontinuierlich oder mit Unterbrechungen appliziert werden sollte, trotz der von Wilke et al. (1998a) vorgestellten Leitiline keine einheitliche Auffasung zwischen den Autoren besteht. Dies schränkt die Vergleichbarkeit zwischen den Studien ein.

\section{Angelegte Drehmomentrichtung}

Der Testdurchlauf sollte in allen drei heuristisch vorgegeben Bewegungsrichtungen durchgeführt werden (s. Kap. 1.4.1). Dies muss durch die entsprechende Konstruktion der Testapparatur ermöglicht sein. Oftmals wurde der Testdurchlauf nicht in allen Bewegungsrichtungen durchgeführt (s Tab. 1), obwohl dies in einigen Fällen durch Modifikation der Testapparatur möglich gewesen wäre (Mansour et al. 2004, Nägerl et al. 2009, Wachowski et al. 2009b, Bozkus et al. 2010, Lazaro et al. 2010, Wachowski et al. 2010, Schulze et al. 2012, Wachowski et al. 2013). Dies wird unter anderem damit begründet, dass in dem jeweiligen Testdurchlauf das Testverfahren validiert werden sollte (Mansour et al. 2004) oder gezielt bestimmte Bewegungsrichtungen untersucht werden sollten (Grassmann et al. 1998, Wachhowski et al. 2009b, Bozkus et al. 2010, Lazaro et al. 2010, Wachowski et al. 2010, Wachowski et al. 2013). In anderen Fällen war die Bewegung des Präparates jedoch durch die Konstruktion der Testapparatur limitiert: Bei Apparaturen bei denen das Drehmoment über kompressive Belastung und Keile appliziert wurde (Cusick et al. 1988, Haher et al. 1991, Rousseau et al. 2006a, Rousseau et al. 2006b, McNally et al. 2012) ist konstruktionsbedingt ohne wesentliche Veränderungen am Aufbau der Versuchsapparatur keine Applikation einer axialen Rotation möglich, da hier nur durch anteriore, posteriore oder laterale Belastung eine Stellung des Präparates vorgegeben wird. Dies stellt unweigerlich eine Limitierung der Versuche dar. McNally et al. unterbanden mit inrer verwendeten Apparatur zudem gezielt Bewegungen außerhalb der Hauptbewegungsebene (Sagittalebene). Damit sollten Bewegungen im Sinne der coupled motion verhindert und die Messgenauigkeit erhöht werden (McNally et al. 2012). Dies entspricht jedoch nicht der physiologischen, dreidimensionalen Bewegung.

\subsection{Vorlast}

Terminologische Abgrenzung der Formen der Vorlastapplikation

In der Literatur wird der Applikation einer Vorlast eine große Bedeutung beigemessen, die zugleich jedoch auch kontrovers diskutiert wird (Patwardhan et al. 1999, Cripton et al. 2000, Kettler et al. 2002, Tawackoli et al. 2004). In der vorliegenden Studie konnte 
gezeigt werden, dass sich bis heute kein einheitliches Verfahren der Vorlastapplikation durchgesetzt hat (s. Kap. 3.3). Terminologisch findet sich in der Literatur dabei eine Abgrenzung zwischen einer follower preload und einer compressive preload. In früheren Studien wurde von einigen Autoren häufig angegeben, eine compressive preload appliziert zu haben (Cusick et al. 1988, Haher et al. 1991, Grassmann et al. 1998, Naderi et al. 1998).

Erst später wurde von Patwardhan et al. (1999) der Begriff follower preload geprägt. Diese Form der Vorlastapplikation sollte im Vergleich zu den bis dato verwendeten Formen der Vorlastapplikation einige Vorteile aufweisen (s.u.). Dabei wird, wie in Kapitel 3.3 ausführlich beschrieben, die Vorlast über seitlich entlang der vermuteten Rotationszentren der jeweiligen Wirbel verlaufende, unter Spannung gesetzte Kabel appliziert (Patwardhan et al. 1999). Es fanden sich einige Studien, die eine follower preload applizierten (Niosi et al. 2006, Sengupta et al. 2011, Patwardhan et al. 2012, Zhu et al. 2012). Eine terminologische Unterteilung in follower preload und compressive prelaod ist jedoch bei genauer Betrachtung der Verwendung der Begriffe in den untersuchten Studien problematisch und kann zu Unklarheiten führen. Denn wie in Kapitel 3.6 gezeigt werden konnte, wird der Begriff compressive preload für zahlreiche verschiedene Formen der Vorlastapplikation verwendet. So beschreibt er beispielsweise eine Vorlastapplikation über einen vertikal ausgerichteten Lastkolben über dem oberen Segment (Cusick et al. 1988, McNally et al. 2012), von Patwardhan et al. auch compressive vertical load genannt, Stauchung durch obere und untere Befestigungsplatten (Haher et al. 1991, Grassmann et al. 1998), Kompression des Präparates (Bozkus et al. 2010) oder durch Kompression des Segmentes durch das Eigengewicht des oberen Befestigungsbehälters (Naderi et al. 1998). Der Begriff compressive preload beschreibt daher lediglich, dass das Präparat komprimiert wurde und lässt daher keine detaillierten Rückschlüsse auf die Art der Vorlastapplikation zu. Auch die follower preload komprimiert das Segment und stellt genau genommen ebenfalls eine compressive preload dar. Lazaro et al. gaben innerhalb einer Studie an, eine compressive follower load bzw. follower load (Lazaro et al. 2010) appliziert zu haben, was die Begriffsunklarheit in der Literatur belegt. Es zeigte sich, dass die Begrifflichkeiten follower preload und compressive preload in der Literatur zwar von einigen Autoren terminologisch unterschieden werden, in der Literatur allerdings die Abgrenzungen unklar sind. Eine detaillierte Beschreibung der Art der Vorlastapplikation ist daher dringend indiziert. 
Als wesentlicher Vorteil der follower preload gilt nach Patwardhan et al., dass Scherkräfte und zusätzliche Beugungsmomente im Vergleich zur compressive preload verhindert werden (Patwardhan et al. 1999). Bei seitlicher Betrachtung nimmt die Wirbelsäule eine S-förmige Gestalt an (s. Kap. 1.2). Wird die Wirbelsäule nun von oben durch einen vertikal ausgerichteten Stempeldruck belastet, wirkt sich die Kompression auf jede einzelne Bewegungseinheit unterschiedlich aus. Es entstehen zusätzliche Beugungsmomente und Scherkräfte, die in vivo nicht vorliegen und schwer zu messen sind. Bei der von Patwardhan et al. vorgestellten follower load folgen die seitlichen Kabel über Führungen dem Verlauf der Wirbelsäule und komprimieren somit jedes einzelne Segment. Daraus resultierte eine internal compressive load (Patwardhan et al. 1999). Durch diesen geführten Verlauf des Kraftvektors, können mit bis zu $1200 \mathrm{~N}$ wesentlich höhere Vorlastgrößen appliziert werden als bei den bis dato verwendeten Formen der Vorlastapplikation (Patwardhan et al. 2003). Zur Applikation einer follower preload ist der Verlauf der Kabelführungen seitlich entlang der einzelnen Rotationszentren des Präparates unbedingte Voraussetzung. Dies erfolgt in vitro jedoch nur näherungsweise (Patwardhan et al. 1999). Des Weiteren ist bekannt, dass die Rotationszentren während einer Bewegung des Präparates keineswegs konstant sind (z. B. Gertzbein et al. 1984, Gertzbein et al. 1985, Samagh et al. 2011 und andere). Hinsichtlich dieser Schwierigkeiten bei der praktischen Umsetzung der Applikation einer follower preload sind dann theoretisch Beugungsmomente und Scherkräfte wiederum nicht auszuschließen. Dieser theoretischen Überlegung gingen Patwardhan et al. (2003) nach und variierten in einem Versuch leicht die Position der Kabelführungen entlang des Segmentes, so dass sich der follower load path veränderte. Die Autoren gaben an, dass sich das Bewegungsausmaß bei den unterschiedlichen Verläufen der seitlichen Kabelführungen nur geringfügig unterschied (Patwardhan et al. 2003). Ein Einfluss der verschiedenen follower load paths auf die Lage und Orientierung einer Rotationsachse wurde dabei jedoch nicht untersucht, so dass nicht ausgeschlossen werden kann, dass die durch die follower preload entstehenden Artefaktmomente und Scherkräfte möglicherweise die Segmentkinematik beeinflussen.

Cripton et al. untersuchten ebenfalls den Effekt verschiedener Formen der Vorlastapplikation auf das biomechanische Verhalten der Wirbelsäule. So testeten sie zusätzlich entstehende Artefaktmomente sowie Scherkräfte. Die Autoren unterschie- 
den dabei zwischen einem ungeführten Verlauf des Kraftvektors der Vorlast, indem das Gewicht am oberen Endes des Präparates befestigt war und auch bei Bewegung des Präparates nur dem Gravitationsvektor folgte sowie anderen Formen der Vorlastapplikation, bei denen der Verlauf des Kraftvektors in verschiedenen Variationen entlang des Präparates geführt wurde. Die Form des ungeführten Kraftvektorverlaufs entspricht dabei der verwendeten Form der Vorlastapplikation von Haberl et al. (2004) und ähnelt denen von anderen Autoren angewandten Verfahren (Mansour et al. 2004, Nägerl et al. 2009, Wachowski et al. 2009a, Wachowski et al. 2009b, Wachowski et al. 2010a, Wachowski et al. 2010b, Takigawa et al. 2010, Wachowski et al. 2013). Folgt das am oberen Ende des Präparates befestigte Gewicht nur dem Gravitationsvektor so kommt es beispielsweise bei einer Flexionsbewegung zu einer Anteriorverlagerung des Kraftvektors. Cripton et al. (2000) konnten zeigen, dass hierbei ein zusätzliches, unerwünschtes Drehmoment induziert wird. Dieses Artefaktmoment entstehe nach Angaben der Autoren auch bei lateraler Beugung sowie Extensionsbewegung. Cripton et al. verwendeten dabei monosegmentale Präparate. Bei polysegmentalen Präparaten seien nach Angaben der Autoren noch höhere Artefaktmomente zu erwarten. Bei axialer Rotationsbewegung wurden hingegen keine zusätzlich entstehenden Artefaktmomente gemessen, da der Kraftvektor der Vorlast hier auch während der Rotation entlang der vertikalen Achse des Präparates verlief. Zusätzliche Scherkräfte wurden bei keiner der Bewegungsrichtungen festgestellt (Cripton et al. 2000). Umgekehrt verhielt es sich jedoch bei Verlaufsformen des Kraftvektors, bei denen der Kraftvektor der Vorlast seitlich entlang des Segmentes geführt wurde. Dies entspricht etwa dem Verlauf des Kraftvektors bei einer follower preload, auch wenn diese von Patwardhan et al. (1999) für polysegmentale Präparate beschrieben wurde. Bei dieser geführten Vorlastapplikation resultierten bei Flexion-Extension sowie lateraler Beugung nach Angaben von Cripton et al. erhöhte Scherkräfte, jedoch keine signifikanten Artefaktmomente. Bei axialer Rotation ergab sich hingegen ein verringertes resultierendes Drehmoment durch wiederum auftretende Artefaktmomente. Bezüglich der Scherkräfte ist anzumerken, dass Rousseau et al. diese mit ihrer verwendeteten Testapparatur gezielt erzeugten, da diese auch in vivo vorkämen (Rousseau et al. 2006a, Rousseau et al. 2006b). Auch Cripton et al. schlossen nicht aus, dass sogenannte Artefaktmomente und Scherkräfte möglicherweise sogar physiologisch sind (Cripton et al. 2000). Da hier jedoch noch nicht genügend Kenntnisse über die in vivo gegebenen Verhältnisse vorliegen (Cripton et al. 2000), sollte zu- 
nächst darauf abgezielt werden, dass die angelegten Drehmomente auch den resultierenden entsprechen und keine zusätzlichen Scherkräfte entstehen, da nur so die Messergebnisse mit den angelegten Parametern korreliert werden können.

Cripton et al. (2000) leiten daraus die Empfehlung ab, dass für Versuchsdurchläufe unter axialer Rotationsbewegung eine ungeführte Form der Vorlastapplikation, also durch freihängende Gewichte, zu verwenden sei, wohingegen für Flexions- und Extensionsbewegungen sowie laterale Beugung eine Vorlast zu applizieren sei, bei der der Kraftvektor dem Verlauf des Präparates folgt, so dass auch bei den jeweiligen Bewegungen eine stets axiale Belastung des Segmentes vorliege. Dies wird jedoch von keinem der Autoren, die den Versuchsdurchlauf sowohl mit angelegtem axialen Drehmoment, Flexions-Extensionsbewegungen bzw. lateralen Beugungsbewegungen durchführten, angewandt. Vielmehr wird, insofern überhaupt eine Vorlast angelegt ist, jeweils eine Form der Vorlastapplikation für alle Bewegungsrichtungen angewandt (s. Tab. 2). Prinzipiell scheint die Applikation einer ungeführten Vorlast technisch leichter zu sein als die Anbringung von seitlichen Kabelführungen an das Präparat entlang der jeweiligen Rotationszentren, wie es die follower preload erfordert. Dies ist aufgrund der manuellen Anbringung der Kabelführungen unter Umständen auch mit Fehlern behaftet.

Bei einigen Versuchen, bei denen eine Vorlast ohne Führung appliziert wurde und Flexions-/Extensions-/ oder laterale Beugungsbewegungen appliziert wurden (Wachowski et al. 2007, Nägerl et al. 2009, Wachowski et al. 2009a, Wachowski et al. 2010a, Takigawa et al. 2010), ist aus den genannten Gründen entsprechend von resultierenden Artefaktmomenten auszugehen, die unter Umständen die Messergebnisse beeinflusst haben könnten. Dabei ist jedoch zu beachten, dass dieser unerwünschte Effekt, wie Cripton et al. feststellten (2000), bei Versuchen mit monosegmentalen Präparaten geringer ausfällt, als dies bei Versuchen mit polysegmentalen Präparaten der Fall wäre.

Bei Versuchen bei denen eine follower preload appliziert wurde, ist entsprechend den Ausführungen von Cripton et al. (2000) bei angelegtem axialen Drehmoment von Artefaktmomenten auszugehen (Niosi et al. 2006, Zhu et al. 2007, Sengupta et al. 2011, Zhu et al. 2012).

Bei der Belastung des Präparates über einen vertikal ausgerichteten Kolben (Cusick et al. 1988, Grassmann et al. 1998, McNally et al. 2012) besteht ein großer Nachteil 
darin, dass vertikale Bewegungen, die im Sinne der coupled motion beispielsweise bei axialer Rotation bestehen, durch das von oben wirkende Gewicht unterbunden werden und wie Grassman et al. (1998) zeigen konnten, vielmehr zu erhöhten internen Kräften führen. Diese entsprechen dann jedoch nicht mehr der angelegten Vorlastgröße, so dass dieser angelegte Parameter letztlich unbekannt ist. Diese Form der Vorlastapplikation entspricht zudem nicht den in vivo vorliegenden Verhältnissen, da das Segment durch das Gewicht geführt wird und nicht, wie beispielsweise bei frei hängenden Gewichten, der physiologischen Bewegungsrichtung folgt. Bei Naderi et al. (1998) handelt es sich letztlich nicht um eine Simulation der angreifenden Muskelkräfte und des Körpergewichtes im Sinne einer Vorlast. Das durch den oberen Befestigungsbehälter resultierende Gewicht ist so gering, dass kaum Einfluss auf die Bewegung des Segmentes zu erwarten ist. Zudem kann es bei einigen Studien ebenfalls zu einer Kraftausübung auf das Präparat durch einen Befestigungsbehälter gekommen sein, dessen Ausmaß jedoch unklar ist.

Wie gezeigt werden konnte, hat die Vorlast einen großen Einfluss auf die Bewegungsstruktur der Präparate. Verschiedene Formen der Vorlastapplikation können entsprechend zu unterschiedlichen Messergebnissen führen. Dies zeigen auch die Tabellen 6 und 7 in Kapitel 3.7.1. und 3.7.2. Hier wird noch einmal aufgezeigt, welchen konkreten Einfluss die Vorlast auf die Lage und Ausrichtung der Rotationsachsen innerhalb des Präparates hat. Um Messergebnisse verschiedener Studien vergleichen zu können, sollte daher darauf geachtet werden, dass auch die Form der Vorlastapplikation gleich war und präzise beschrieben wurde (Cripton et al. 2000). Versuchsbeschreibungen, in denen dies nicht erfolgte (s. Tab. 2), vernachlässigen schließlich einen wichtigen Parameter, so dass keine valide Einschätzung der Versuchsparameter möglich ist.

Versuche in denen keine Vorlast verwendet wurde (s. Tab. 2), stellen letztlich keine Simulation physiologischer Verhältnisse dar, was von Brasiliense et al. entsprechend auch als Limitierung angegeben wird (Brasiliense et al. 2011). Versuche, in denen gezielt keine Vorlast appliziert wurde (Dugailly et al. 2010, Dugailly et al. 2011, Dugailly et al. 2013, Schulze et al. 2012), erhöhen zwar durch die Reduktion einflussnehmender Parameter die Reproduzierbarkeit der Messergebnisse, allerdings handelt es sich nicht um physiologische Bedingungen.

Tabelle 8 zeigt für alle Studien, die eine Vorlast applizierten, ob gemäß der dargestell- 
ten Überlegungen mit Artefaktmomenten oder Scherkräften zu rechnen ist. Studien, in denen keine Vorlast appliziert wurde, sind aus Gründen der Übersichtlichkeit nicht angeführt. Eine Tabelle über die Art der Vorlastapplikation für alle Studien findet sich in Kapitel 3.3 (Tab. 2). Tabelle 8 zeigt dabei, dass es keine Form der Vorlastapplikation gibt, bei der eine Rückwirkung auf das Präparat auszuschließen ist.

Tab. 8 Mögliche Artefaktmomente/ Scherkräfte für die jeweilige Studien.

\begin{tabular}{|c|c|c|c|}
\hline Autor & $\begin{array}{l}\text { Art der Vorlastapplika- } \\
\text { tion }\end{array}$ & $\begin{array}{l}\text { Dreh- } \\
\text { moment- } \\
\text { richtung }\end{array}$ & $\begin{array}{l}\text { Rückwirkung der VL auf } \\
\text { Messergebnisse }\end{array}$ \\
\hline $\begin{array}{l}\text { Bozkus et al. } \\
2010\end{array}$ & $\begin{array}{l}\text { compressive load über } \\
\text { Stauchung des Präpara- } \\
\text { tes durch Riemen (s. } \\
\text { Text) }\end{array}$ & FI-Ex & $\begin{array}{l}\text { Artefaktmomente durch ungenaue } \\
\text { Ausführung des Prinzips } \\
\text { der follower load s. Text }\end{array}$ \\
\hline Cusick et al 1988 & $\begin{array}{l}\text { compressive preload } \\
\text { über vertikalen Kolben } \\
\text { (s.Text) }\end{array}$ & $\mathrm{FI}$ & $\begin{array}{l}\text { möglicherweise Bewegung im } \\
\text { Sinne der coupled motion } \\
\text { unterbunden }\end{array}$ \\
\hline $\begin{array}{l}\text { Grassmann et al. } \\
1998\end{array}$ & $\begin{array}{l}\text { compressive load über } \\
\text { Stauchung des Präpara- } \\
\text { tes durch starre Befesti- } \\
\text { gungsplatten (s.Text) }\end{array}$ & AR & $\begin{array}{l}\text { nach Angaben der Autoren: Bewegung } \\
\text { im Sinne der coupled motion unterbun- } \\
\text { den }\end{array}$ \\
\hline Haher et al. 1991 & $\begin{array}{l}\text { compressive load über } \\
\text { vertikale Höhenverände- } \\
\text { rung der Befestigungs- } \\
\text { platten (s. Text) }\end{array}$ & FI-Ex & $\begin{array}{l}\text { möglicherweise Bewegung im } \\
\text { Sinne der coupled motion unterbunden }\end{array}$ \\
\hline $\begin{array}{l}\text { Lazaro et al. } \\
2010\end{array}$ & $\begin{array}{l}\text { compressive follower } \\
\text { load über Stauchung des } \\
\text { Präparates durch Riemen } \\
\text { (s. Text) }\end{array}$ & FI-Ex & $\begin{array}{l}\text { möglicherweise Artefaktmomente durch } \\
\text { ungenaue Ausführung des Prinzps der } \\
\text { follower load s. Text }\end{array}$ \\
\hline $\begin{array}{l}\text { Mansour et al. } \\
2004\end{array}$ & $\begin{array}{l}\text { VL-Applikation über frei } \\
\text { hängende Gewichte }\end{array}$ & AR & $\begin{array}{l}\text { geringe Artefaktmomente bei coupled } \\
\text { motion und bei Lateralflexion bzw. Flexi- } \\
\text { on/Extension }\end{array}$ \\
\hline $\begin{array}{l}\text { McNally et al. } \\
2012\end{array}$ & $\begin{array}{l}\text { compressive load über } \\
\text { vertikale Krafteinwirkung } \\
\text { auf obere Befestigungs- } \\
\text { platte (s. Text) }\end{array}$ & $\mathrm{FI}$ & $\begin{array}{l}\text { möglicherweise Bewegung im } \\
\text { Sinne der coupled motion unterbunden }\end{array}$ \\
\hline Naderi et al. 1998 & $\begin{array}{l}\text { compressive preload } \\
\text { durch Eigengewicht des } \\
\text { oberen Befestigungsbe- } \\
\text { hälters (s. Text) }\end{array}$ & AR, FI-Ex, LB & $\begin{array}{l}\text { Gering bei coupled motion, VL mit 5,2 N } \\
\text { zu unphysiologisch }\end{array}$ \\
\hline Niosi et al. 2006 & follower preload & AR, FI-Ex, LB & $\begin{array}{l}\text { mögliche Artefaktmomente bei AR, ge- } \\
\text { ringe Scherkräfte bei FI-Ex/ LB }\end{array}$ \\
\hline $\begin{array}{l}\text { Rousseau et al. } \\
2006 a \text {, } \\
2006 b\end{array}$ & $\begin{array}{l}\text { compressive preload (s. } \\
\text { Text) über vertikal ein- } \\
\text { wirkende Kraft auf obere } \\
\text { Befestigungsplatte (s. } \\
\text { Text) }\end{array}$ & FI-Ex, LB & $\begin{array}{l}\text { Scherkräfte in diesem Fall vom Autor } \\
\text { erwünscht, möglicherweise Bewegung im } \\
\text { Sinne der coupled motion unterbunden }\end{array}$ \\
\hline $\begin{array}{l}\text { Sengupta et al. } \\
2011\end{array}$ & follower preload & AR, FI-Ex, LB & $\begin{array}{l}\text { mögliche Artefaktmomente bei AR, } \\
\text { möglicherweise Scherkräfte bei FI-Ex/ LB }\end{array}$ \\
\hline $\begin{array}{l}\text { Takigawa et al. } \\
2010\end{array}$ & $\begin{array}{l}\text { compressive preload } \\
\text { über von oberer Befesti- } \\
\text { gungsplatte herabhän- } \\
\text { gende Gewichte (s. Text) }\end{array}$ & AR, FI-Ex, LB & $\begin{array}{l}\text { mögliche Artefaktmomente bei FI-Ex/ LB } \\
\text { jedoch nur geringfügig, da monosegmen- } \\
\text { tale Präparate verwendet wurden }\end{array}$ \\
\hline
\end{tabular}




\begin{tabular}{|l|l|l|l|}
\hline $\begin{array}{l}\text { Wachowski et al. } \\
\mathbf{2 0 0 7} \\
\text { Wachowski et al. } \\
\mathbf{2 0 0 9 a}\end{array}$ & $\begin{array}{l}\text { VL-Applikation über frei } \\
\text { hängende Gewichte }\end{array}$ & AR, FI-Ex, LB & $\begin{array}{l}\text { mögliche Artefaktmomente bei FI-Ex/ LB } \\
\text { jedoch nur geringfügig, da monosegmen- } \\
\text { tale Präparate verwendet wurden }\end{array}$ \\
\hline $\begin{array}{l}\text { Wachowski et al. } \\
\mathbf{2 0 0 9 b}\end{array}$ & $\begin{array}{l}\text { VL-Applikation über frei } \\
\text { hängende Gewichte }\end{array}$ & AR & $\begin{array}{l}\text { minimale Artefaktmomente bei coupled } \\
\text { motion möglich }\end{array}$ \\
\hline $\begin{array}{l}\text { Wachowski et al. } \\
\mathbf{2 0 1 0 a}\end{array}$ & $\begin{array}{l}\text { VL-Applikation über frei } \\
\text { hängende Gewichte }\end{array}$ & AR, FI-Ex & $\begin{array}{l}\text { mögliche Artefaktmomente bei FI-Ex } \\
\text { jedoch nur geringfügig, da monosegmen- } \\
\text { tale Präparate verwendet wurden }\end{array}$ \\
\hline $\begin{array}{l}\text { Wachowski et al. } \\
\mathbf{2 0 1 0 b}\end{array}$ & $\begin{array}{l}\text { VL-Applikation über frei } \\
\text { hängende Gewichte } \\
\text { minimale Artefaktmomente bei coupled } \\
\text { motion möglich }\end{array}$ & AR & $\begin{array}{l}\text { minimale Artefaktmomente bei coupled } \\
\text { motion möglich }\end{array}$ \\
\hline $\begin{array}{l}\text { Wachowski et al. } \\
\mathbf{2 0 1 3}\end{array}$ & $\begin{array}{l}\text { VL-Applikation über frei } \\
\text { hängende Gewichte }\end{array}$ & AR & $\begin{array}{l}\text { mögliche Artefaktmomente bei AR, } \\
\text { möglicherweise Scherkräfte bei FI-Ex/ LB }\end{array}$ \\
\hline Zhu et al. $\mathbf{2 0 0 7}$ & follower preload & $\begin{array}{l}\text { mögliche Artefaktmomente bei AR, mög- } \\
\text { licherweise Scherkräfte bei Fl-Ex/ LB }\end{array}$ \\
\hline Zhu et al. $\mathbf{2 0 1 2}$ & follower preload & AR, FI-Ex, LB &
\end{tabular}

AR axiale Rotation, FI-Ex Flexion-Extension, LB laterale Beugung, VL Vorlast

\section{Vorlastgröße}

Auch bezüglich der Vorlastgröße finden sich zahlreiche Kontroversen. Patwardhan et al. führten als einen wesentlichen Nachteil der compressive preload an, dass nur geringe Vorlastgrößen applizierbar sind, die weit unter den physiologischen Werten liegen sollten, die wiederum bei einigen Tausend Newton angenommen wurden (Patwardhan et al. 1999). Ein positiver Effekt der follower preload sei, dass nach Patwardhan et al. (2003) Vorlastgrößen von bis zu $1200 \mathrm{~N}$ applizierbar sind. Interessanterweise wird jedoch von keinem der Autoren, die eine follower preload nach den Vorgaben von Patwardhan et al. applizierten (Niosi et al. 2006, Zhu et al. 2007, Sengupta et al. 2011, Zhu et al. 2012), eine Vorlastgröße dieser Größenordnung angelegt (s. Tab. 2).

Da die Vorlast ansetzende Muskelkräfte und das Körpergewicht simulieren soll, sind für zervikale Segmente hier geringere Größen anzulegen als für thorakale oder lumbale. So applizierten Wachowski et al. in einer Studie für zervikale Segmente bis zu $70 \mathrm{~N}$ und für lumbale Segmente bis zu $300 \mathrm{~N}$ (Wachowski et al 2009a). Adams gab an, dass die durchschnittliche Kompressionskraft, die auf lumbale Segmente einwirkt, etwa $500 \mathrm{~N}$ im Stehen und $750 \mathrm{~N}$ im Sitzen entspricht. Beim Aufheben eines Gegenstandes können je nach Gewicht des Gegenstandes Kompressionskräfte von bis zu $6000 \mathrm{~N}$ wirken (Adams 1995). 
Einige Autoren applizierten eine verhältnismäßig geringe Vorlast (Haberl et al. 2003, Mansour et al. 2004, Sengupta et al. 2011, Wachowski et al. 2013). Haberl et al. begründeten dies damit, dass dadurch das Bewegungsausmaß nicht zu sehr reduziert wird. Dadurch können Veränderungen des Bewegungsausmaßes während des Versuchdurchlaufes unter veränderten äußeren Bedingungen, reproduzierbarer gemessen werden. Die Autoren gaben an, dass es sich dabei jedoch um eine Limitierung der Studie, gemessen an möglichst physiologischen Bedingungen handelt (Haberl et al. 2004). Dies zeigt, dass zu niedrige und zu hohe Werte die Messergebnisse beeinflussen können, da zu niedrige Werte das Bewegungsausmaß größer und zu hohe Werte das Bewegungsausmaß geringer werden lassen. Zu hohe Werte können zudem zu Beschädigungen des Präparates führen, die vermutlich jedoch weit über den in den vorliegenden Studien verwendeten Werten liegen. Bezüglich der verwendeten Vorlastgrößen ist also auch hier festzustellen, dass sich in der Literatur keine einheitlichen Angaben finden.

\subsection{Verwendete Präparate}

\section{Menschliche Präparate/ tierische Präparate}

Es fand sich nur eine Studie (Schulze et al. 2012), in der keine menschlichen Präparate getestet wurden. Prinzipiell erlauben ermittelte Ergebnisse aus Testverfahren mit tierischen Präparaten nahezu keine Rückschlüsse auf die biomechanischen und kinematischen Verhältnisse bei Menschen. Sie können jedoch zur Validierung eines Testverfahrens dienen. Die Verwendung von menschlichen Präparaten erweist sich zum einen aus ethischen Gesichtspunkten als problematisch. Zum anderen ist die Zahl der zur Verfügung stehenden Spenderpräparate sehr gering. Für die Untersucher erweist es sich daher als schwierig geeignete menschliche Präparate zu erhalten (Wilke et al. 1996). Bei Versuchen mit einer geringen Anzahl an Präparaten z. B. nur einem Präparat (Cripton et al. 2001, Dugailly et al. 2013) ist im Vergleich zu Testverfahren mit mehreren Präparaten, z. B. 47 Präparaten (Seligman et al. 1984) die Reproduzierbarkeit der Ergebnisse entsprechend stark eingeschränkt. Zudem bleiben interindividuelle Unterschiede unberücksichtigt. Aus Testergebnissen mit nur sehr wenigen Präparaten sollten demnach keine allgemeingültigen Aussagen über die Kinematik und Biomechanik der Wirbelsäule getroffen werden. Um solche Aussagen treffen zu können, sollten als weiteres Kriterium nur intakte, das heißt pathologisch unauffällige Präparate Verwendung finden. Da einige Autoren jedoch keine Angaben dazu machten, ob sie die Präparate zuvor auf pathologische Auffälligkeiten untersucht 
zu haben, können unphysiologische Testbedingungen nicht sicher ausgeschlossen werden (Cripton et al. 2001, Kettler et al. 2004, Niosi et al. 2006, Zhu et al. 2007, Dugailly et al. 2011, Sengupta et al. 2011, McNally et al. 2012, Schulze et al. 2012, Zhu et al. 2012). Ausnahmen stellen gemäß der Zielstellung der jeweiligen Studie Versuche dar, in denen gezielt auch pathologische Zustände untersucht werden sollten (Seligman et al. 1984). Gertzbein et al. und Seligman et al. konnten zeigen, dass sich die Lage der center of rotations bei Segmenten mit degenerativen Erscheinungen signifikant von der Position der center of rotations bei unauffälligen Präparaten unterschied (Gertzbein et al. 1984, Gertzbein et al. 1985). Bei Analyse der Messergebnisse muss immer auch Kenntnis über den anatomischen Zustand des Präparates bestehen. Auch hier müssen entsprechend Versuchsbeschreibungen, die keine Angaben zum Zustand der Präparate machten als unzureichend gelten (z. B. Cripton et al. 2001, Kettler et al. 2004, Niosi et al. 2006, Zhu et al. 2007, Dugailly et al. 2010, Dugailly et al. 2011, Dugailly et al. 2013, Sengupta et al. 2011, Samagh et al. 2011, McNally et al. 2012, Schulze et al. 2012, Zhu et al. 2012 und andere). Ähnliche Feststellungen sind auch bezüglich des Alters der Präparate zu treffen. Brasiliense et al. verwendeten Präparate mit einem Alter von bis zu 92 Jahren und vermuteten, dass diese in ihrer Mobilität eingeschränkt seien (2011). Somit sind dann auch Veränderungen der Biomechanik nicht auszuschließen (Takigawa et al. 2010), da degenerative Veränderungen der Testobjekte sehr wahrscheinlich sind. Entsprechend sind Kenntnisse über das Alter der Präparate, dem Zustand des Präparates sowie dem untersuchten Level von hoher Bedeutung.

\section{Segmenthöhe}

Dass der größte Teil der Autoren lumbale Segmente in den Mittelpunkt der Betrachtung stellt, ist aufgrund der hier häufig vorliegenden pathologischen Erscheinungen in Zusammenhang zu bringen.

\section{Präparation}

An dieser Stelle soll auf die Sensitivität der Testobjekte auf die verschiedenen Formen der Präparation während der Untersuchungsvorgänge der einzelnen Studien hingewiesen werden. Ein umfassender Vergleich der jeweils verschiedenen Formen soll, gemäß der Zielstellung der vorliegenden Studie und der in den meisten Fällen ungenauen Angaben in den Studien (s. Kapitel 3.4) bezüglich der genannten Untersuchungskriterien, nicht erfolgen. 
Bezüglich der Präparation der Testobjekte zeigte sich als generelles Prinzip, dass Weichgewebe entfernt wurden und ligamentäre sowie knöcherne Strukturen erhalten blieben. Diese Vorgehensweise geht mit den generellen Empfehlungen zur Präparation von Testsegmenten einher (Wilke et al. 1998a).

\section{Mono/Polysegmental}

Der Vorteil von polysegmentalen Präparaten ist nach Haher et al., dass der Einfluss der Testapparatur geringer ist als bei Versuchen mit monosegmentalen Präparaten (Haher et al. 1992). Jedoch sollte die Applikation der Drehmomente generell rückwirkungsfrei sein und die Testapparatur keinen Einfluss auf die Bewegungsstruktur des Segmentes haben. Nur so kann eine physiologische Bewegung sichergestellt werden. Wenn die Testapparatur Einfluss auf die Bewegungsstruktur des Präparates hat, bedeutet dies zugleich auch, dass die auf die Präparate wirkenden Parameter unbekannt sind. Messergebnisse sind dementsprechend wenig aussagekräftig. Zwar weist die Wirbelsäule in vivo ebenfalls eine polysegmentale Struktur auf, jedoch können polysegmentale Präparate bei In-vitro-Versuchen auch erhebliche Nachteile aufweisen. So bringt die Applikation eines Drehmomentes an polysegmentale Präparate bei monosegmentaler Messung eine Ungenauigkeit bezüglich der im zu testenden Segment wirkenden Parameter mit sich. Die resultierenden Ergebnisse stellen vielmehr ein Ergebnis des gesamten Präparates dar, als dass die biomechanischen und kinematischen Verhältnisse des zu testenden Segmentes nachvollzogen werden können. Dies ist bei Applikation eines Drehmomentes an monosegmentale Präparate bei monosegmentaler Messung im gleichen Segment einfacher. Dies setzt jedoch eine bedingungslos rückwirkungsfreie Applikation des Drehmomentes voraus. Da die Wirbelsäule in vivo jedoch aus mehreren Segmenten besteht, wäre eine Applikation des Drehmomentes an ein polysegmentales Präparat bei Messung der Bewegungsstruktur in jedem einzelnen Segment, quasi einer polysegmentalen Messung, ideal. Dieses Verfahren wird von Cagli et al. (2004) und Detwiler et al. (2003) durchgeführt, jedoch verwendeten die Autoren eine Apparatur, die keine rückwirkungsfreien Drehmomente applizierte (s.o). (Detwiler 2003, Cagli et al. 2004).

\subsection{Messapparaturen}

Die Messergebnisse werden wesentlich durch die Genauigkeit der Messapparatur bestimmt. So können mittels einer zweidimensionalen Messmethodik (Seligman 1984, 
Molnar 2006) entsprechend keine dreidimensionalen Messdaten errechnet werden. Angaben zur Orientierung der Rotationsachse sind dann nicht möglich. Die Messergebnisse haben daher keine kinematische Bedeutung.

Bei der Genauigkeit der Messapparatur kann zwischen einer räumlichen und einer zeitlichen Auflösung unterschieden werden. Hier fanden sich erhebliche Unterschiede (s. Tab. 4). Um eine Bewegung kontinuierlich aufzeichnen zu können müssen differentiell kleine Bewegungsstrecken in differentiell kleinen Zeitabständen aufgezeichnet werden (s. Kapitel 1.5.2). Hierbei zeigten einige mechanische Messmethodiken vergleichsweise höhere Auflösungen im Vergleich zu dem häufig verwendeten optischen Messsystem (Optotrak 3020). Ein Vorteil der optischen Messmethodik ist, dass sie, durch Anbringen weiterer Marker, auch für polysegmentale Messungen erweitert werden kann. Wohingegen einige mechanische Messverfahren (Wachowski et al. 2007, Nägerl et al. 2009, Wachowski et al. 2007, Wachowski et al. 2009a, Wachowski et al. 2009b, Wachowski et al. 2010, Wachowski et al. 2010b, Wachowski et al. 2013) hier eine Limitierung aufweisen, da die mechanischen Taster aufgrund ihrer Länge nur für monosegmentale Messungen verwendet werden können (Wachowski 2014 persönliche Mitteilung). Bei optischen Verfahren verringert sich hingegen die zeitliche Auflösungsfähigkeit in Abhängigkeit zur Anzahl der verwendeten Marker (Oxland 2014). Daher ist die zeitliche Auflösungsfähigkeit bei polysegmentalen Präparaten geringer als bei monosegmentalen Präparaten. Gemessen an der zeitlichen und räumlichen Auflösungsfähigkeit zeigten sich einige mechanische Messverfahren jedoch gegnüber den optischen Verfahren als deutlich überlegen (s. Tab. 4).

Die in Kapitel 1.5.2 formulierte Forderung, die Lagemessung der Körper in differentiell kleinen Intervallen zu messen, wird also nur in wenigen Studien erfüllt (Mansour et al. 2004, Wachowski et al. 2007, Nägerl et al. 2009, Wachowski et al. 2007, Wachowski et al. 2009a, Wachowski et al. 2009b, Wachowski et al. 2010, Wachowski et al. 2010b, Sengupta 2011, Wachowski et al. 2013). Sengupta et al. gaben dabei an, eine kontinuierliche Messung vorgenommen zu haben. Genaue Angaben zur Auflösung fanden sich nicht (Sengupta et al. 2011).

Die in den anderen Studien erfolgte Aufzeichnung der Bewegung stimmt daher nicht mit der tatsächlichen Bewegungsstruktur der Wirbel überein.

\subsection{Angaben zu Rotations-/Schraubachsen der untersuchten Studien}

Neben Angaben zur Ausrichtung ist auch eine differentiell kleine Messauflösung nötig 
(s. Kapitel 1.5.2), so dass die Rotations-/Schraubachsen kontinuierlich aufgezeichnet werden können. Die in Kapitel 1.5.2 gestellte Forderung der Messung der Bewegung in differentiell kleinen Intervallen wird nur von wenigen Autoren erfüllt (Mansour et al. 2004, Wachowski et al. 2007, Wachowski et al. 2009a, Nägerl et al. 2009, Wachowski et al.2009b, Wachowski et al.2010a, Wachowski et al.2010b, Wachowski et al. 2013). In den untersuchten Studien resultierten Angaben von nur einer Lokalisation der Rotations-/Schraubachse pro Bewegungsausmaß bis hinzu Lokalisationsangaben von bis zu 800 pro Bewegungsausmaß (s. Tab. 5 S. 56)).

In Kapitel 3.6.1 konnte gezeigt werden, dass die Rotations-/Schraubachse in allen Segmenten, abhängig von Vorlast, angelegtem Drehmoment und untersuchtem Level wandert. Mansour et al. (2004) konnten hierzu zeigen, dass der Schnittpunkt der Schraubachse mit der Transversalebene (bei axialer Rotation) durch die Wanderung der Schraubsachse einen Pfad darstellt, der eine Strecke von bis zu $60 \mathrm{~mm}$ zurücklegte (Rastpolkurve). Gibt man nun, wie in einigen Fällen, mehrere ermittelte Lokalisationen der Rotationsachse als eine Durchschnittsposition an (Naderi et al. 1998, Chen et al. 2001, Crawford et al. 2002, Cagli et al. 2004, Bozkus et al. 2010, Lazaro et al. 2010), bedeutet dies entsprechend eine starke Vereinfachung der Messergebnisse, die keine valide Einschätzung der kinematischen Bewegungsstruktur in Abhängigkeit der Wirbelsäule erlaubt.

Um die Kinematik der Wirbelsäule valide beschreiben zu können, müssen neben Angaben zur Lokalisation auch Angaben zur Ausrichtung der Rotations-/Schraubachse erfolgen. Dies wird jedoch nicht von allen Autoren erfüllt (s. Tab. 5).

Nach den Gesetzen der Segmentkinematik kann die Bewegung eines Körpers jedoch nur dann suffizient beschrieben werden, wenn der Bewegungsbereich zwischen den zwei Positionen differentiell klein ist (s. Kap. 1.5.2). In den Studien, in denen eine annähernd differentiell kleine Messauflösung vorlag (s. Tab. 4), zeigte sich eine stetige Wanderung der Rotations-/Schraubachse, was die Notwendigkeit der Aufzeichnung der Bewegung in differentiell kleinen Intervallen belegt (s.u.). Bei einer statisch verharrenden Achse wären auch die Richtungsvektoren stets konstant. Dies war jedoch in keiner der Studien, die die Wanderung der Rotations-/Schraubachse aufzeichnen konnten der Fall. Die Achsen zeigten eine stetige Wanderung und Änderung ihrer Ausrichtung (s.u.) (s. Tab. 6 und 7). Ausnahmen stellen Flexions- Extensionsbewegungen dar. Hier zeigte sich eine nahezu konstante Position der Achse und sich 
kaum ändernde Orientierungen der Rotationsachse (s. Tab. 6 und 7, s.u.).

Neben den verschiedenen Intervallgrößen, für die eine Rotationsachse berechnet wurde, zeigte sich eine generelle Begriffsunklarheit bezüglich der Rotations/Schraubachsen. So wurden zum Teil in der gleichen Studie unterschiedliche Bezeichnungen für die Achsen angegeben. So gaben Molnar et al. (2006) an, zum einen eine rotational axis errechnet zu haben, zum anderen führten sie den Begriff center of rotation (COR) an. Cagli et al. (2004) verwendeten die Begriffe finite axis of rotation, axis of rotation, helical axis of motion (HAM) sowie instantaneous axis of rotation (IAR). Hier wird die Begriffsunklarheit besonders deutlich. Broc et al. bezeichneten die ermittelte Rotationsachse als IAR bzw. FHA. Dabei scheint vor allem der Begriff IAR in der Literatur umstritten zu sein. Bozkus et al. und Lazaro et al. gaben hierzu an, dass es sich bei der IAR um ein Äquivalent zur FHA handelt bei dem jedoch keine Translation beachtet wird (Bozkus et al. 2010, Lazaro et al. 2010). Auch Rousseau et al. bezeichneten die IAR als Rotationsachse, um die nur Rotation und keine Translation stattfinden solle (Rousseau et al. 2006a). Sengupta et al. gaben hingegen an, dass auch bei der IAR Translationsbewegungen berücksichtigt werden (Sengupta et al. 2011). Noch deutlicher zeigt sich die Begriffsunklarheit bei Seligman et al., die angaben, mehrere center of rotations errechnet zu haben und so den Verlauf einer IAR darstellen zu können (Seligman et al. 1984). Jedoch ist ein COR eine zweidimensionale Angabe und die IAR dreidimensional. Da die Autoren jedoch dreidimensionale Bewegungen gezielt unterbunden haben, können keine dreidimensionalen Angaben gemacht werden. Auch der Begriff instantaneous (deutsch. unmittelbar) ist hier auf Grund der zu geringen zeitlichen und räumlichen Auflösung der Apparatur falsch verwendet, da es sich bei der Bewegungsaufzeichnung nicht um die unmittelbare Wiedergabe der Bewegung handelt.

Cripton et al. (2001) gaben an, eine HAM berechnet zu haben. Die Rotationsachsen konnten hingegen in Intervallen von $0,5^{\circ}$ angegeben werden, so dass für eine axiale Rotation nach rechts 6-12 HAM resultierten (Cripton et al. 2001). Gleiches gilt auch für Haberl et al., die $10 \mathrm{HAM} / \mathrm{ROM}$ (Haberl et al. 2004) und Grassmann et al., die 8 HAM/ ROM berechneten. Kettler et al. (2004), Dugailly et al. (2010, 2011, 2013), und Schulze et al. (2012) teilten die Bewegung ebenfalls in grobe Intervalle nannten die errechnete Schraubachse jedoch finite helical axis, was nicht mit der Terminologie der anderen untersuchten Studien einhergeht (s.o.). 
Es ist daher festzustellen, dass bezüglich der Beschreibung der Rotations/Schraubachsen keine einheitliche Terminologie vorliegt.

Ein weiterer Kritikpunkt ist, dass einige Autoren nur einen Teilbereich des gesamten Bewegungsausmaßes untersuchten, da sie den Bereich der EZ (s. Kap.1.5.3) für biomechanisch relevant hielten. Jedoch ist dieser Bereich allein kinematisch ohne Bedeutung (s. Kap. 1.5.3). Es sollte vielmehr über den gesamten Bereich des ROM die Bewegung kinematisch erfasst werden. So berechneten Detwiler et al. eine finite axis of rotation jeweils vom Bereich des Endes der Neutralen Zone bis zum maximalen Bewegungsausmaß. Zudem resultierten somit nur zwei axes of rotation, was der Forderung einer kontinuierlichen Aufzeichnung der Bewegung in differentiell kleinen Bewegungsintervallen (s. Kap. 1.5.2) nicht gerecht wird.

Einige Autoren legten an das Segment Drehmomente in drei verschiedenen Richtungen an, so dass theoretisch eine Rotations-/Schraubachse in drei Bewegungsrichtungen ermittelt werden konnte. Die Rotations-/Schraubachse wurde dann jedoch oftmals nur für eine der angelegten Bewegungsrichtungen ermittelt, die anderen Bewegungsrichtungen wurden vernachlässigt. Dabei handelte es sich in den meisten Fällen um Flexions-Extensionsbewegungen (Naderi et al. 1998, Chen et al. 2001, Detwiler et al. 2003, Cagli et al. 2004, Bozkus et al. 2010, Lazaro et al. 2010). Einige Autoren begründeten dies damit, dass die Bewegungen außerhalb der Hauptbewegungsebene, also Bewegungen im Sinne der coupled motion, für axiale Rotationen bzw. laterale Beugung zu groß waren und es so zu Ungenauigkeiten der Messung gekommen wäre (Naderi et al. 1998, Cagli et al. 2004), bzw. eine zweidimensionale Darstellung einer dreidimensionalen Bewegung nicht möglich gewesen wäre, so dass die Lage der Rotationsachse nur für Flexions- Extensionsbewegung ermittelt wurden, da hier hauptsächlich zweidimensionale Bewegungen stattfänden (Chen et al. 2001). Es wurde zwar erkannt, dass die Bewegung zu einem großen Anteil auch außerhalb der jeweiligen Hauptbewegungsebene stattfindet, auf Grund der Fehleranfälligkeit der Messmethodiken war es jedoch nicht möglich diese darzustellen. Die Angaben werden damit artifiziell auf nur eine Bewegungsrichtung reduziert. Dies stellt entsprechend eine Limiterung der Messverfahren dar. Die Ergebnisse dieser Messungen geben nicht die physiologischen Verhältnisse wieder.

Die Validierungstests, die in einigen Studien durchgeführt wurden, zeigten auf, dass die Lagebestimmung einer Rotations-/Schraubachse zum Teil mit erheblichen Fehlern 
behaftet war. So können Abweichungen in der Genauigkeit der Angabe der Lokalisation der Achse in der Größe von einigen Millimetern (s. Tab. 5) nicht akzeptiert werden, wenn man sich bewusst macht, dass die Strecke, die eine Rotations/Schraubachse in einer Ebene zurücklegt, selbst nur einige Millimeter beträgt (Gregersen und Lucas 1967).

Da jedoch nur wenige Studien einen solchen Validierungstest durchführten, sind die Werte der einzelnen Studien schlecht vergleichbar. Es muss daher die Forderung formuliert werden, dass in allen In-vitro-Tests Validierungstests zur Feststellung der Genauigkeit des Testvorgangs durchgeführt werden sollten.

\subsection{Vergleich der Lokalisation ermittelter Rotations-/Schraubachsen ausgewähl- ter Studien}

Der direkte Vergleich der Lokalisation der Rotations-/Schraubachse zeigt, dass die verschiedenen Messmethoden zum Teil völlig unterschiedlichen Angaben führten. Dabei hatte die Art der Vorlastapplikation einen entscheidenden Einfluss auf die Lage der Rotations-/ Schraubachse (s. Tab. 6 und 7). Dies hebt noch einmal die Bedeutung der Vorlast hervor, die - wie gezeigt werden konnte - oftmals nicht appliziert wurde (s. Tab. 2).

Zusammengefasst zeigte sich dabei eine geringe Wanderung der Rotations/Schraubachse bei Flexion-Extension. Das heißt, dass der Unterschied zwischen der Messung der Rotations-/Schraubachse in differentiell kleinen Intervallen und Messungen mit nur einem Intervall hier geringer ist als bei anderen Bewegungsrichtungen. So wurde bei lateraler Beugung in einigen Fällen eine ausgeprägte Wanderung der Schraubachse von 15-40 mm beschrieben (s. Tab. 6). Ähnliche Angaben gelten auch für zervikale Segmente. Hier war die Wanderung jedoch weniger stark ausgeprägt (s. Tab. 6).

Die auffälligsten Unterschiede zwischen Angaben zu einer ortsfesten und einer in nahezu differentiell kleinen Intervallen aufgelösten Rotations-/Schraubachse zeigten sich bei axialen Rotationsbewegungen in lumbalen Segmenten. Hier zeigte sich, dass die Achse eine große Wanderung vollzog: In verschiedenen Untersuchungen wurde übereinstimmend festgestellt, dass die Schraubachse Wanderungsstrecken von bis zu $60 \mathrm{~mm}$ in der Transversalebene von einem Facettengelenk zum anderen zurücklegte (z. B. Mansour et al. 2004, Sengupta et al. 2011, Wachowski et al. 2009a und andere). Hier wird deutlich, dass die in Kapitel 1.5.2 formulierte Forderung, die Be- 
wegung in differentiell kleinen Intervallen aufzuzeichnen und wiederzugeben, von hoher Bedeutung ist, da andernfalls wichtige Informationen über die Bewegungsstruktur unbeachtet blieben. Eine Beschreibung einer ortsfesten Rotationsachse (z. B. Niosi et al. 2006, Zhu et al. 2007, Takigawa et al. 2010 und andere) stellt somit keine valide Beschreibung der Bewegungsstruktur dar, da hier die Wanderung der Rotations/Schraubachse vernachlässigt wird.

Bezüglich der Orientierung der Rotations-/Schraubachse zeigte sich verallgemeinert, dass die Orientierung, bis auf einige Ausnahmen, für zervikale und lumbale Segmente nahezu parallel zum angelegten Drehmoment war (s. Tab. 6 und 7). Wenn es zu Abweichungen dieser Ausrichtung kam, wurde dies mit interindividuellen Unterschieden oder durch Bewegungen im Sinne der coupled motion erklärt. So zeigte sich bei Cripton et al. (2001) eine $45^{\circ}$-Neigung der Rotationsachse zur Transversalebene bei paralleler Ausrichtung zur Sagittalebene, eine gekoppelte Bewegung aus lateraler Beugung und axialer Rotation. Eine reine axiale Bewegung hätte eine kranio-kaudal ausgerichtete Rotationsachse zur Folge. Bei einer reinen lateralen Beugung würde die Rotation um eine anterior-posteriorausgerichtete Rotations-/Schraubachse stattfinden. Die sich verändernde Ausrichtung der Rotations-/Schraubachse liefert somit weitere wichtige Erkenntnisse über die kinematische Bewegungsstruktur der Wirbelsäule. Jedoch wurden oftmals nur Angaben zur Lokalisation, nicht aber zur Orientierung der Achse gemacht (s. Tab. 5).

Es konnte gezeigt werden, dass Studien, in denen die Rotations-/Schraubachse sowohl in ihrer Ausrichtung als auch für differentiell kleine Bewegungsintervalle beschrieben werden konnte, mehr Informationen über die Kinematik liefern, als Studien, bei denen nur eine Position der Rotations-/Schraubachse ohne Wanderung und Orientierung angegeben wurde. Es zeigte sich, dass nur wenige Autoren (Mansour et al. 2004, Wachowski et al. 2007, Nägerl et al. 2009, Wachowski 2009a, Wachowski 2009b, Wachowski et al. 2010a, Wachowski et al. 2010b, Wachowski et al. 2013) in der Lage waren, die Bewegung der Rotations-/ Schraubachse für differentiell kleine Intervalle (s. Kap. 1.5.2) zu beschreiben. Weiterhin zeigt sich, dass einige Autoren in der Lage waren, die Bewegung der Wirbelsegmente in differentiell kleine Abschnitte kinematisch korrekt aufzulösen. Angaben zur Rotationsachse wie bei Cusick et al. (1988), die die Lage der Rotationsachse nur grob anatomischen Regionen zuordneten, sind ungenau und erlauben keine Rückschlüsse auf die kinematische Bewegungsstruktur der Wirbelsegmente. 


\subsection{Angaben zum Bewegungsausmaß und Gelenkkräften in den untersuchten Studien}

In Kapitel 1.5.3 wurde bereits gezeigt, dass das ROM keine kinematische Bedeutung hat. Dennoch werden hierzu von fast allen Autoren Angaben gemacht. Dies ist damit in Zusammenhang zu bringen, dass es technisch leicht zu erfassen ist. Es enthält jedoch keine Angaben zur Bewegungsstruktur, sondern steht vielmehr in direktem Zusammenhang zum jeweils angelegten Drehmoment. In den Fällen, in denen das ROM weiter in eine neutrale bzw. elastische oder Stiff- bzw. Lax zone unterteilt wurde, findet eine weitere Interpretation der Kennlinien statt. Die Ermittlung dieser Unterteilungen erweist sich jedoch als untersucherabhängig. Das ROM hängt dabei von den Testbedingungen ab. So ist bei polysegmentalen Messungen ein höheres ROM zu erwarten als bei Messungen in nur einem Segment, da mehrere Segmente eine größere Bewegung zurücklegen als wenige Segmente. Wie von einigen Autoren angegeben, haben die Vorlastgröße und die Vorlastposition einen Einfluss auf das ROM (Haberl et al. 2004, Niosi et al 2006, Nägerl et al. 2009, Wachowski et al. 2009a). Dies unterstreicht zumindest die Bedeutung einer Vorlast auf das biomechanische Verhalten der Segmente, wenngleich auch die Bewegungsstruktur anhand des ROM nicht nachvollzogen werden kann. Neben der Vorlast hat auch das Drehmoment wesentlichen Einfluss auf das ROM. So ist bei zu geringen Drehmomentgrößen davon auszugehen, dass nicht das volle ROM erreicht wird, wohingegen sich das Segment bei zu hohen Drehmomenten über das physiologische ROM hinaus bewegt, bis der als plastische Zone bezeichnete Bereich der irreversiblen Zerstörung des Präparates erreicht wird (s. Abb. 7 S. 20). Dies unterstreicht die Erkenntnis, dass das ROM keine valide Beschreibung der Bewegungsstruktur zulässt. In der vorliegenden Analyse konnte dennoch gezeigt werden, dass die Ermittlung dieser Kennlinien in der Literatur weit verbreitet ist, obwohl sie keinen Rückschluss auf die Segmentkinematik liefern.

Die Ermittlung der Gelenkkräfte hat oftmals den Nachteil, dass dazu die Gelenkkapseln geöffnet werden müssen. Takigawa et al. gaben hierzu an, dass dies jedoch nur einen geringfügigen Effekt auf die Biomechanik der Segmente hatte (Takigawa et al. 2010). Auch hier gilt, dass Messungen der Gelenkkräfte nützliche Informationen bezüglich der Belastung der einzelnen Segmente liefern können, da so zum Beispiel eine Überbelastung benachbarter Segmente nach Fusion (adjacent segment disease) untersucht werden kann (Takigawa et al. 2010). Kenntnisse über die physiologische Bewegung des Segmentes liefern solche Messdaten jedoch nicht. Da wie bei 
Rousseau et al. (2006a, 2006b) die Messsensoren sogar die Hälfte der Gelenkflächen einnehmen, ist hier vielmehr von einem negativen Einfluss der Messsensoren auf die physiologische Segmentkinematik auszugehen. 


\section{Zusammenfassung}

Ziel der vorliegenden Arbeit war, die in der Literatur präsentierten Verfahren zur Ermittlung biomechanischer und kinematischer Daten der Wirbelsäule bei In-vitroVersuchen hinsichtlich ihrer Methodik und Messergebnisse zu analysieren. Es erfolgte eine Literaturrecherche in den medizinischen Datenbanken PUBMED und WEB OF SCIENCE. Als Suchbegriffe dienten die aus der Biomechanik und Kinematik der Wirbelsäule bekannten Stichworte. Hier wurden insbesondere die aus der Literatur bekannten verschiedenen Bezeichnungen der Schraub-/Rotationsachsen verwendet.

Dabei zeigte sich in Bezug auf die Versuchsmethodik, dass bei allen Untersuchungen starke Vereinfachungen der in vivo vorkommenden Verhältnisse angewandt wurden. Von zahlreichen Autoren wurden ansetzende Muskeln sowie das Körpergewicht durch eine Vorlast simuliert. Die angewandten Verfahren ermöglichten eine gute Reproduzierbarkeit und Kontrollierbarkeit, entsprachen jedoch nur bedingt der Physiologie. Auch bezüglich der Drehmomentapplikation zeigten sich unterschiedliche Verfahren, wobei jedoch keine der Formen Artefaktmomente bzw. zusätzliche Scherkräfte gänzlich ausschlossen. Da bekannt ist, dass ein Körper sich in sechs Freiheitsgraden bewegen kann, sollte diese Anforderung auch an die Testapparaturen gestellt werden. Jedoch zeigte sich, dass nur in sehr wenigen Fällen diese Anforderung erfüllt wird. Nur wenige Autoren konnten eine von der Testapparatur unabhängige, rückwirkungsfreie Bewegung generieren. Bezüglich der angelegten Parameter (Vorlastgröße, Drehmomentgröße) zeigte sich, dass keine einheitlichen Größen angelegt wurden und somit ein Vergleich der Messergebnisse der Studien untereinander erschwert wird.

Bezüglich der Messergebnisse zeigte sich ebenfalls wenig Übereinstimmung zwischen den verschiedenen Studien. Einige Autoren berichteten von einer ortsfesten Rotationsachse, die während des gesamten Bewegungsausmaßes nicht wandere. In anderen Fällen wurde erkannt, dass die Rotations-/Schraubachse während der Bewegung wandert, jedoch wurde das Bewegungsausmaß nur in grobe Intervalle unterteilt. Nur wenige Autoren konnten die kontinuierliche Wanderung der Achse erfassen und in quantitativer wie auch qualitativer Form darstellen. In den Fällen, in denen die Vorlast in Größe und Position variiert wurde, zeigte sich zum Teil ein erheblicher Einfluss dieser Parameter auf die kinematische Bewegungsstruktur des Präparates. 
Eine Ermittlung der Lage und Orientierung der Achse in Intervallen lieferte abhängig vom Zeitpunkt der Messung jeweils unterschiedliche Messergebnisse. In den Fällen, in denen in Intervallen gemessen wurde, zeigte sich für jedes Intervall eine zum vorherigen Intervall veränderte Lokalisation. Daher muss von einer in Ausmaß und Art variierenden Wanderung der Rotations-/Schraubachse ausgegangen werden. Allgemein wird von den Autoren erkannt, dass keine Bewegung nur plan in einer Ebene stattfindet. Es handelt sich vielmehr um eine komplexe dreidimensionale Bewegung, was in der Literatur mit dem Begriff coupled motion beschrieben wird. Bedingt durch die Gelenkmorphologie kommt es entsprechend der dreidimensionalen Bewegung des Segmentes auch zu Änderungen der Ausrichtung der Rotations-/Schraubachse. Die Morphologie wird wird jedoch von einigen Autoren vernachlässigt, da die technischen Möglichkeiten der reproduzierbaren Darstellung der physiologischen Segmentbewegung begrenzt sind. In einigen Fällen werden sogar bewusst Bewegungsrichtungen nicht untersucht, da hier die komplexen Bewegungen messtechnisch nicht erfasst werden können. Änderungen der Ausrichtung der Rotations-/Schraubachse können nur bei Messungen dargestellt werden, die die Bewegung in Intervallen aufnehmen. Diese Anforderung wird in den überwiegenden Fällen jedoch nicht erfüllt.

Zusammenfassend ist von validen Messmethoden zu fordern, dass differentiell kleine Bewegungsintervalle erfasst werden. Hierzu ist eine hohe zeitliche und örtliche Auflösung notwendig. Die angelegten Kräfte, die die Bewegung generieren, müssen die rückwirkungsfreie, physiologische Bewegung des Testobjektes ermöglichen und aufzeichnen. Ideal wären Messapparaturen, die den Zug der einzelnen Muskeln und das Gewicht des Körpers simulieren und variieren können. Weiterhin sollten die Versuchsbedingungen reproduzierbar und kontrollierbar sein.

In der vorliegenden Arbeit konnte gezeigt werden, dass keine aktuell verfügbare Apparatur alle Anforderungen erfüllen kann. Allein die korrekte Erfassung der Kinematik wird in wenigen Studien gewährleistet. 


\section{Literaturverzeichnis}

Adams MA (1995): Mechanical testing of the spine. An appraisal of methodology, results, and conclusions. Spine 20, 2151-2156

Adams MA, Dolan P (1991): A technique for quantifying the bending moment acting on the lumbar spine in vivo. J Biomech 24, 117-126

Aumüller G, Aust G, Doll A, Engele J, Kirsch J, Mense S, Reißig D, Salvetter J, Schmidt W, Schmitz F: Anatomie (Duale Reihe). 2. Auflage; Thieme Verlag, Stuttgart 2010

Bertagnoli R, Yue JJ, Fenk-Mayer A, Eerulkar J, Emerson JW (2006):

Treatment of symptomatic adjacent-segment degeneration after lumbar fusion with total disc arthroplasty by using the prodisc prosthesis: a prospective study with 2-year minimum follow up. J Neurosurg Spine 4 , 91-97

Beutler WJ, Clavenna AL, Gudipally M, Moldavsky M, Khalil S (2012): A biomechanical evaluation of spacer with integrated plate for treating adjacent-level disease in the subaxial cervical spine. Spine $\mathrm{J} \underline{12}, 585-589$

Bockermann V: Kinematik des Halswirbelsäulen- Segments C5/6. Biomechanische Analyse seines Bewegungsmusters bei physiologischen Standardbelastungen. Med. Diss. Greifswald 2004

Bozkus H, Senoglu M, Baek S, Sawa AG, Ozer AF, Sonntag VK, Crawford NR (2010): Dynamic lumbar pedicle screw-rod stabilization: in vitro biomechanical comparison with standard rigid pedicle screw-rod stabilization. J Neurosurg Spine 12, 183-189

Brasiliense LB, Lazaro BC, Reyes PM, Dogan S, Theodore N, Crawford NR (2011): Biomechanical contribution of the rib cage to thoracic stability. Spine $36,1686-$ 1693

Broc GG, Crawford NR, Sonntag VK, Dickman, CA (1997): Biomechanical effects of transthoracic microdiscectomy. Spine 22, 605-612

Cagli S, Chamberlain RH, Sonntag VK, Crawford NR (2004): The biomechanical effects of cervical multilevel oblique corpectomy. Spine 29, 1420-1427

Caravaggi P, Chaudary S, Uko L, Chen L, Khamsi B, Vives M (2013): A novel design for application of pure moments in-vitro: application to the kinematic analysis of the cervical spine. J Biomech 뜨, 1221-1224

Chen TY, Crawford NR, Sonntag VK, Dickman CA (2001): Biomechanical effects of progressive anterior cervical decompression. Spine 26, $6-13$

Cossette JW, Farfan HF, Robertson GH, Wells RV (1971): The instantaneous center of rotation of the third lumbar intervertebral joint. J Biomech $\underline{4}, 149-153$

Crawford NR (2006): Technical Note: Determining and Displaying the Instantaneous Axis of Rotation of the Spine. Spine 13, 1033-1041 
Crawford NR (2014): persönliche Mitteilung per Email (15.06.2014)

Crawford NR, Brantley AG, Dickman CA, Koeneman EJ (1995): An apparatus for applying pure nonconstraining moments to spine segments in vitro. Spine $\underline{20}$, 2097-2100

Crawford NR, Peles JD, Dickman CA (1998): The spinal lax zone and neutral zone: measurement techniques and parameter comparisons. J Spinal Disord 11, 416-429

Crawford NR, Duggal N, Chamberlain RH, Park SC, Sonntag VK, Dickman CA (2002): Unilateral cervical facet dislocation: injury mechanism and biomechanical consequences. Spine $\underline{27}, 1858-1864$

Cripton PA, Bruehlmann SB, Orr TE, Oxland TR, Nolte LP (2000): In vitro axial preload application during spine flexibility testing: towards reduced apparatusrelated artefacts. J Biomech $\underline{33}, 1559-1568$

Cripton PA, Sati M, Orr TE, Bourquin Y, Dumas GA, Nolte LP (2001): Animation of in vitro biomechanical tests. J Biomech $\underline{34}, 1091-1096$

Cunningham BW, Gordon JD, Dmitriev AE, Hu N, McAfee PC (2003): Biomechanical evaluation of total disc replacement arthroplasty: an in vitro human cadaveric model. Spine 28, 110-117

Cusick JF, Yoganandan N, Pintar F, Myklebust J, Hussain H (1988): Biomechanics of cervical spine facetectomy and fixation techniques. Spine $\underline{13}, 808-812$

Detwiler PW, Spetzler CB, Taylor SB, Crawford NR, Porter RW, Sonntag VK (2003): Biomechanical comparison of facet-sparing laminectomy and Christmas tree laminectomy. J Neurosurg 99, 214-220

Dickman CA, Crawford NR, Paramore CG (1996): Biomechanical characteristics of C1-2 cable fixations. J Neurosurg $\underline{85}, 316-322$

Dugailly PM, Sobczak S, Sholukha V, Van Sint Jan S, Salvia P, Feipel V, Rooze M (2010): In vitro 3D-kinematics of the upper cervical spine: helical axis and simulation for axial rotation and flexion extension. Surg Radiol Anat $\underline{32}, 141-$ 151

Dugailly PM, Sobczak S, Moiseev F, Sholukha V, Salvia P, Feipel V, Rooze M, Van Sint Jan S (2011): Musculoskeletal modeling of the suboccipital spine: kinematics analysis, muscle lengths, and muscle moment arms during axial rotation and flexion extension. Spine $\underline{36}, 413-422$

Dugailly PM, Sobczak S, Lubansu A, Rooze M, Jan SS, Feipel V (2013): Validation protocol for assessing the upper cervical spine kinematics and helical axis: An in vivo preliminary analysis for axial rotation, modeling, and motion representation. J Craniovertebr Junction Spine 4 4, 10-15 
Fick R: Handbuch der Anatomie und Mechanik der Gelenke unter Berücksichtigung der bewegenden Muskeln. Anatomie der Gelenke. Bd.1; G. Fischer, Jena 1910

Gertzbein SD, Holtby R, Tile M, Kapasouri A, Chan KW, Cruickshank B (1984): Determination of a locus of instantaneous centers of rotation of the lumbar disc by moire fringes. A new technique. Spine $\underline{9}, 409-413$

Gertzbein SD, Seligman J, Holtby R, Chan KH, Kapasouri A, Tile M, Cruickshank B (1985): Centrode patterns and segmental instability in degenerative disc disease. Spine 10, 257-261

Goertzen DJ, Lane C, Oxland TR (2004): Neutral zone and range of motion in the spine are greater with stepwise loading than with a continuous loading protocol. An in vitro porcine investigation. J Biomech $\underline{37}, 257-261$.

Grassmann S, Oxland TR, Gerich U, Nolte LP (1998): Constrained Testing Conditions Affect the Axial Rotation Response of Lumbar Functional Spinal Units. Spine 23, 1155-1162

Gregersen GG, Lucas DB (1967): An in- vivo study of the axial rotation of the human thoracolumbar spine. J Bone Joint Surg $\underline{49-A}, 247$

Günther H-P, Maier B, Marzi I, Pizanis A, Pohlemann T, Seifert J: Wirbelsäule. In: Scharf HP, Rüter A, Pohlemann T, Marzi I, Kohn D, Günther KP (Hrsg.): Orthopädie und Unfallchirurgie. 2. Auflage; Urban \& Fischer, München 2011, 595-681

Haberl H, Cripton PA, Orr TE, Beutler T, Frei H, Lanksch WR, Nolte LP (2004): Kinematic response of lumbar functional spinal units to axial torsion with and without superimposed compression and flexion/extension. Eur Spine $\mathrm{J} \underline{13}$, 560-566

Haher TR, Bergman M, O'Brien M, Felmly WT, Choueka J, Welin D, Chow G, Vassiliou A (1991): The effect of the three columns of the spine on the instantaneous axis of rotation in flexion and extension. Spine $\underline{16}, 312-318$

Haher TR, O'Brien, M, Felmly WT, Welin D, Perrier G, Choueka J, Devlin V, Vassiliou A, Chow $G$ (1992): Instantaneous axis of rotation as a function of the three columns of the spine. Spine 17, 149-154

Junghanns H: Die Wirbelsäule in Forschung und Praxis. Bd. 75; Hippokrates- Verlag, Stuttgart 1977

Kaner T, Sasani M, Oktenoglu T, Ozer AF (2010): Dynamic stabilization of the spine: a new classification system. Turk Neurosurg 20, 205-215

Kettler A, Marin F, Sattelmayer G, Mohr M, Mannel H, Durselen L, Claes L, Wilke HJ (2004): Finite helical axes of motion are a useful tool to describe the threedimensional in vitro kinematics of the intact, injured and stabilised spine. Eur Spine J 13, 553-559 
Kotani Y, Cunningham BW, Abumi K, Dmitriev AE, Hu N, Ito M, Shikinami Y, McAfee PC, Minami A (2006): Multidirectional flexibility analysis of anterior and posterior lumbar artificial disc reconstruction: in vitro human cadaveric spine model. Eur Spine J 15, 1511-1520

Lazaro BC, Brasiliense LB, Sawa AG, Reyes PM, Theodore N, Sonntag VK, Crawford NR (2010): Biomechanics of a novel minimally invasive lumbar interspinous spacer: effects on kinematics, facet loads, and foramen height. Neurosurgery $\underline{66}, 126-132$

Mansour M: Die biomechanische Funktion der Articulationes zygapophysiales der Lendenwirbelsäule. Med. Diss. Göttingen 2001

Mansour M, Spiering S, Lee C, Dathe H, Kalscheuer AK, Kubein-Meesenburg D, Nägerl $H$ (2004): Evidence for IHA migration during axial rotation of a lumbar spine segment by using a novel high-resolution 6D kinematic tracking system. J Biomech $\underline{37}$, 583-592

McNally D, Naylor J, Johnson S (2012): An in vitro biomechanical comparison of Cadisc- $L$ with natural lumbar discs in axial compression and sagittal flexion. Eur Spine J 21, 612-617

Molnar S, Mano S, Kiss L, Csernatony Z (2006): Ex vivo and in vitro determination of the axial rotational axis of the human thoracic spine. Spine $\underline{31}, 984-991$

Naderi S, Crawford NR, Song GS, Sonntag VK, Dickman CA (1998): Biomechanical comparison of $\mathrm{C} 1-\mathrm{C} 2$ posterior fixations. Cable, graft, and screw combinations. Spine $\underline{23}, 1946-1955$

Nägerl H (2014): persönliche Mitteilung

Nägerl $H$, Kubein-Meesenburg D, Cotta $H$, Fanghänel J, Rossow A, Spiering S (1995): Biomechanische Prinzipien in Diarthrosen und Synarthrosen Teil IV: Zur Mechanik der Wirbelsäule im Lendenbereich. Eine Pilotstudie. Z. Orthop. $133,481-491$

Nägerl H, Hawellek T, Lehmann A, Hubert J, Saptschak J, Dorner J, Raab BW, Fanghanel J, Kubein-Meesenburg D, Wachowski MM (2009): Non-linearity of flexion-extension characteristics in spinal segments. Acta Bioeng Biomech 11, 3-8

Niosi CA, Zhu QA, Wilson DC, Keynan O, Wilson DR, Oxland TR (2006):

Biomechanical characterization of the three-dimensional kinematic behaviour of the Dynesys dynamic stabilization system: an in vitro study. Eur Spine J $\underline{15}$, 913-922

Oxland TR (2014): persönliche Mitteilung per Email (27.06.2014)

Oxland TR, Panjabi MM, Lin RM (1994): Axes of motion of thoracolumbar burst fractures. J Spinal Disord $\underline{7}, 130-138$ 
Panjabi MM (1992): The stabilizing system of the spine. Part II. Neutral zone and instability hypothesis. J Spinal Disord $\underline{5}$, 390-396

Panjabi MM, Brand RA Jr., White AA 3rd (1976): Mechanical properties of the human thoracic spine as shown by three-dimensional load-displacement curves. J Bone Joint Surg Am $\underline{58}, 642-652$

Panjabi MM, Krag MH, Dimnet JC, Walter SD, Brand RA (1984): Thoracic spine centers of rotation in the sagittal plane. J Orthop Res 1 , 387-394

Panjabi MM, Tech D, Brown M, Lindahl S, Irstam L, Hermens M (1988): Intrinsic disc pressure as a measurement of integrity of the lumbar spine. Spine $\underline{13}, 913-7$

Patwardhan AG, Havey RM, Meade KP, Lee B, Dunlap B (1999): A follower load increases the load-carrying capacity of the lumbar spine in compression. Spine 24, 1003-1009

Patwardhan AG, Havey RM, Carandang G, Simonds J, Voronov LI, Ghanayem AJ, Meade KP, Gavin TM, Paxinos O (2003): Effect of compressive follower preload on the flexion-extension response of the human lumbar spine. $J$ Orthop Res $\underline{21}$, 540-546

Patwardhan AG, Tzermiadianos MN, Tsitsopoulos PP, Voronov LI, Renner SM, Reo ML, Carandang G, Ritter-Lang K, Havey RM (2012): Primary and coupled motions after cervical total disc replacement using a compressible six-degreeof-freedom prosthesis. Eur Spine J 211, 618-629

Platzer W: Taschenatlas Anatomie, Band 1: Bewegungsapparat. 9. Auflage; Thieme Verlag, Stuttgart 2005

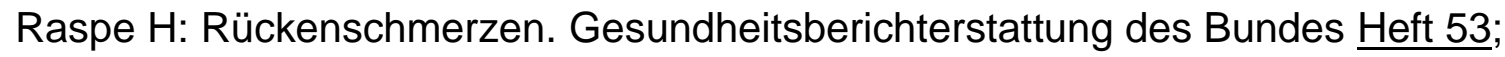
Robert Koch-Institut, Berlin 2012

Rousseau MA, Bradford DS, Bertagnoli R, Hu SS, Lotz JC (2006a): Disc arthroplasty design influences intervertebral kinematics and facet forces. Spine J $\underline{6}, 258-$ 266

Rousseau MA, Bradford DS, Hadi TM, Pedersen KL, Lotz JC (2006b): The instant axis of rotation influences facet forces at $\mathrm{L} 5 / \mathrm{S} 1$ during flexion/extension and lateral bending. Eur Spine J 15, 299-307

Samagh SP, Rosen CD, Otarodifard K, Kornswiet M, Palmer G, Lee TQ (2011): New method for determining apparent axial center of rotation of lumbar and thoracic spine segments. J Rehabil Res Dev 4요, 587-596

Sanan A, Rengachary SS (1996): The history of spinal biomechanics. Neurosurgery $\underline{39}, 657-668$

Schiebler TH, Korf HW: Anatomie. 10. Auflage; Steinkopff Verlag, Heidelberg 2007 
Schmidt CO, Raspe H, Pfingsten M, Hasenbring M, Basler HD, Eich W, Kohlmann T (2007): Back pain in the German adult population: prevalence, severity, and sociodemographic correlates in a multiregional survey. Spine $\underline{32}, 2005-2011$

Schulze M, Hartensuer R, Gehweiler D, Holscher U, Raschke MJ, Vordemvenne T (2012): Evaluation of a robot-assisted testing system for multisegmental spine specimens. J Biomech 노, 1457-1462

Schünke M: Funktionelle Anatomie: Topographie und Funktion des Bewegungssystems. 1.Auflage; Thieme Verlag, Stuttgart 2000

Seligman JV, Gertzbein SD, Tile M, Kapasouri A (1984): Computer analysis of spinal segment motion in degenerative disc disease with and without axial loading. Spine $\underline{9}, 566-573$

Sengupta DK, Demetropoulos CK, Herkowitz HN (2011): Instant axis of rotation of L4-5 motion segment--a biomechanical study on cadaver lumbar spine. J Indian Med Assoc 109, 389-390, 392-393, 395

Smith TJ (1991): In vitro spinal biomechanics. Experimental methods and apparatus. Spine $\underline{16}, 1204-1210$

Spielmann H: In-Vitro-Methoden. In: Marquardt H, Schäfer S, Spielmann H: Lehrbuch der Toxikologie. 2.Auflage; Wissenschaftliche Verlagsgesellschaft $\mathrm{mbH}$, Stuttgart 2004, 1197-1208.

Statistisches Bundesamt: Gesundheit Krankheitskosten. Fachserie 12, Reihe 7.2; Wiesbaden 2010

Szpalski M, Gunzburg R, Mayer M (2002): Spine arthroplasty: a historical review. Eur Spine J 11, 65-84

Takigawa T, Espinoza Orias AA, An HS, Gohgi S, Udayakumar RK, Sugisaki K, Natarajan RN, Wimmer MA, Inoue N (2010): Spinal kinematics and facet load transmission after total disc replacement. Spine $\underline{35}, 1160-1166$

Voß R: Leibeswand. In: Bommas-Ebert U, Teubner P, Voß R: Kurzlehrbuch Anatomie und Embryologie. 3. Auflage; Thieme Verlag, Stuttgart 2011, 135-159

Wachowski MM (2014): persönliche Mitteilung

Wachowski MM, Ackenhausen A, Dumont C, Fanghanel J, Kubein-Meesenburg D, Nägerl H (2007): Mechanical properties of cervical motion segments. Arch Mech Eng 느, 5-15

Wachowski MM, Mansour, M, Lee C, Ackenhausen A, Spiering S, Fanghanel J, Dumont C, Kubein-Meesenburg D, Nägerl H (2009a): How do spinal segments move? J Biomech 42, 2286-2293

Wachowski MM, Hubert, J, Hawellek T, Mansour M, Dorner J, Kubein-Meesenburg D, Fanghanel J, Raab BW, Dumont BC, Nägerl H (2009b): Axial rotation in the lumbar spine following axial force wrench. J Physiol Pharmacol $\underline{60}, 61-64$ 
Wachowski MM, Hawellek T, Hubert J, Lehmann A, Mansour M, Dumont C, Dorner J, Raab BW, Kubein-Meesenburg D, Nägerl H (2010a): Migration of the instantaneous axis of motion during axial rotation in lumbar segments and role of the zygapophysial joints. Acta Bioeng Biomech 12, 39-47

Wachowski MM, Mansour M, Hawellek T, Kubein-Meesenburg D, Hubert J, Nägerl H (2010b): Parametric control of the stiffness of lumbar segments. Strain $\underline{47}$, 281-287

Wachowski MM, Wagner M, Weiland J, Dorner J, Raab BW, Dathe H, Gezzi R, Kubein-Meesenburg D, Nägerl H (2013): Does total disc arthroplasty in C3/C4segments change the kinematic features of axial rotation? J Biomech $\underline{46}$, 1739-1745

Wenig C M, Schmidt C O, Kohlmann T, Schweikert B (2009): Costs of back pain Germany. Eur J Pain $\underline{13}$, 280-286

Wilder DG, Pope MH, Seroussi RE, Dimnet J, Krag MH (1989): The balance point of the intervertebral motion segment: an experimental study. Bull Hosp Jt Dis Orthop Inst $\underline{49}, 155-169$

Wilke HJ, Krischak S, Claes LE (1996): Formalin fixation strongly influences biomechanical properties of the spine. J Biomech $\underline{29}$, 1629-1631

Wilke HJ, Wenger K, Claes L (1998a): Testing criteria for spinal implants: recommendations for the standardization of in vitro stability testing of spinal implants. Eur Spine J $\underline{7}, 148-154$

Wilke HJ, Jungkunz B, Wenger K, Claes LE (1998b): Spinal segment range of motion as a function of in vitro test conditions: effects of exposure period, accumulated cycles, angular-deformation rate, and moisture condition. Anat $\operatorname{Rec} \underline{251}, 15-19$

Zhu Q, Larson CR, Sjovold SG, Rosler DM, Keynan O, Wilson DR, Cripton PA, Oxland TR (2007): Biomechanical evaluation of the Total Facet Arthroplasty System: 3-dimensional kinematics. Spine 32, 55-62

Zhu Q, Itshayek E, Jones CF, Schwab T, Larson CR, Lenke LG, Cripton PA (2012): Kinematic evaluation of one- and two-level Maverick lumbar total disc replacement caudal to a long thoracolumbar spinal fusion. Eur Spine J 21, 599-611 


\section{Lebenslauf}

Am 29. Juni 1988 wurde ich, Conrad Raschke, als zweites Kind von Gabriele Raschke (geb. Zeidler) und Dr. Dipl. Ing. Helmut Raschke in Magdeburg geboren. Nach dem Besuch der Grundschule von 1995-1999 folgte der Wechsel auf das Norbertusgymnasium Magdeburg. Dort erlangte ich im Juli 2007 die allgemeine Hochschulreife. Anschließend legte ich den Zivildienst bei dem Malteser Hilfsdienst Magdeburg von September 2007 bis Mai 2008 ab. Zum Wintersemester 2008/2009 begann ich das Zahnmedizinstudium an der Georg-August-Universität Göttingen, das ich im November 2013 mit dem Staatsexamen erfolgreich beendete. Seit Oktober 2014 arbeite ich als Assistenzzahnarzt. 